Studi di archivistica, bibliografia, paleografia 5

\title{
Oltre le mostre
}

a cura di

Mauro Brunello, Valentina De Martino

e Maria Speranza Storace

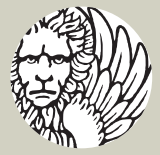

\section{Edizioni}

Ca'Foscari

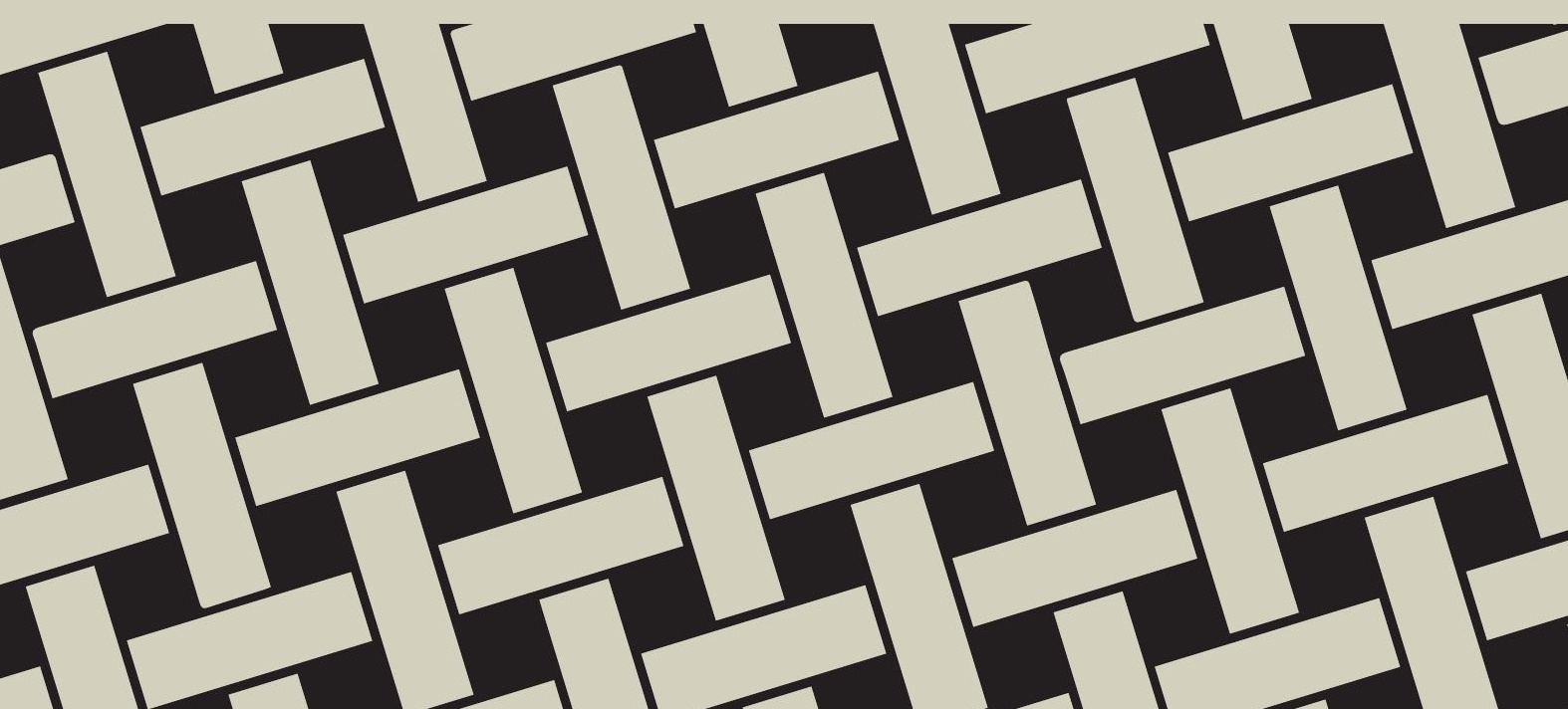



Oltre le mostre

\section{Studi di archivistica, bibliografia e paleografia}

Serie diretta da

Flavia De Rubeis

Dorit Raines

5

Edizioni

Ca'Foscari 


\section{Studi di archivistica, bibliografia, paleografia}

\section{Direttore | General editor}

Flavia De Rubeis (Università Ca' Foscari Venezia, Italia)

Dorit Raines (Università Ca' Foscari Venezia, Italia)

\section{Comitato scientifico |Advisory board}

Jos Biemans (Universiteit van Amsterdam, Nederland)

Giorgetta Bonfiglio Dosio (Università Ca' Foscari Venezia, Italia)

Lorena Dal Poz (Regione del Veneto, Italia)

Vicente García Lobo (Universidad de León, España)

Nicoletta Giovè (Università degli Studi di Padova, Italia)

Neil Harris (Università degli Studi di Udine, Italia)

Marilena Maniaci (Università degli Studi di Cassino, Italia)

Giulio Negretto (Regione del Veneto, Italia)

Marco Pozza (Università Ca’ Foscari Venezia, Italia)

Andreina Rigon (Regione del Veneto, Italia)

Richard Sharpe (University of Oxford, UK)

Melania Zanetti (Università Ca' Foscari Venezia, Presidente AICRAB)

\section{Direzione e redazione}

Dipartimento di Studi Umanistici

Palazzo Malcanton Marcorà

Dorsoduro 3484/D

30123 Venezia

\footnotetext{
e-ISSN 2610-9093

ISSN 2610-9875
}

URLhttps://edizionicafoscari.unive.it/it/edizioni/collane/studi-di-archivistica-bibliografia-paleografia/ 


\section{Oltre le mostre}

a cura di

Mauro Brunello, Valentina De Martino

e Maria Speranza Storace

Venezia

Edizioni Ca' Foscari - Digital Publishing 2020 
Oltre le mostre

Mauro Brunello, Valentina De Martino, Maria Speranza Storace (a cura di)

(c) 2020 Mauro Brunello, Valentina De Martino, Maria Speranza Storace per il testo (c) 2020 Edizioni Ca' Foscari - Digital Publishing per la presente edizione

\section{(c) (i)}

Quest'opera è distribuita con Licenza Creative Commons Attribuzione 4.0 Internazionale This work is licensed under a Creative Commons Attribution 4.0 International License

OPENAS

Qualunque parte di questa pubblicazione può essere riprodotta, memorizzata in un sistema di recupero dati o trasmessa in qualsiasi forma o con qualsiasi mezzo, elettronico o meccanico, senza autorizzazione, a condizione che se ne citi la fonte.

Any part of this publication may be reproduced, stored in a retrieval system, or transmitted in any form or by any means without permission provided that the source is fully credited.

Edizioni Ca’ Foscari - Digital Publishing

Fondazione Università Ca' Foscari Venezia | Dorsoduro 3246 | 30123 Venezia

http://edizionicafoscari.unive.it|ecf@unive.it

la edizione dicembre 2020

ISBN 978-88-6969-466-0 [ebook]

ISBN 978-88-6969-467-7 [print]

La pubblicazione di questo volume è stata sostenuta economicamente dall'Associazione Italiana dei Conservatori e Restauratori degli Archivi e delle Biblioteche (AICRAB).

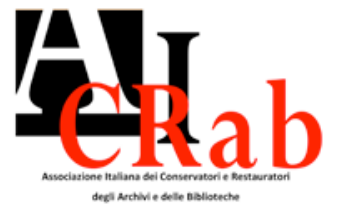

Oltre le mostre / Mauro Brunello, Valentina De Martino, Maria Speranza Storace (a cura di) 1. ed. - Venezia: Edizioni Ca' Foscari - Digital Publishing, 2020. - 138 pp.; 23 cm. - (Studi di archivistica, bibliografia, paleografia; 5). - ISBN 978-88-6969-467-7

URL https://edizionicafoscari.unive.it/en/edizioni/libri/978-88-6969-467-7/

DOI http://doi.org/10.30687/978-88-6969-466-0 


\section{Sommario}

Introduzione. Oltre le mostre

Melania Zanetti

Patrimonio archivistico e librario e nuove strategie di marketing (digitale)

Come ripensare la comunicazione

Anna Busa

I luoghi della memoria 2.0

Strategie di promozione social e digital della biblioteca e dell'archivio

Michela Corsini

I codici si mostrano

Esperienze nella Biblioteca Malatestiana

Paola Errani

Valorizzare i fondi d'autore

Anna Manfron

Archiporto: dalle carte d'archivio alle carte da gioco Sofia Stefani, Elisa Di Liberato

L'alluvione del 1966 e la Biblioteca Nazionale Un 'safari' della memoria

Alessandro Sidoti

\section{La cultura in fuga}

San Lorenzo Escape e la storia raccontata per enigmi

Daniele Ferraiuolo

Oltre le mostre per promuovere i patrimoni documentari 
Etica della collaborazione e responsabilità

Per una nuova gestione, valorizzazione e trasmissione

del patrimonio culturale

Giorgio Busetto

Non di sole mostre vivono gli archivi

Micaela Procaccia

L'Archivio della Pontificia Università Gregoriana (APUG)

e la costruzione di un oltre

Martín M. Morales 
Etica della collaborazione e responsabilità

Per una nuova gestione, valorizzazione e trasmissione

del patrimonio culturale

Giorgio Busetto

Non di sole mostre vivono gli archivi

Micaela Procaccia

L'Archivio della Pontificia Università Gregoriana (APUG)

e la costruzione di un oltre

Martín M. Morales 


\section{Introduzione. Oltre le mostre}

Melania Zanetti

Università Ca' Foscari Venezia, Italia

Questa pubblicazione raccoglie gli Atti del seminario Oltre le mostre. Proposte per una diversa valorizzazione del patrimonio archivistico e librario organizzato dall'Associazione Italiana dei Conservatori e Restauratori degli Archivi e delle Biblioteche (AICRAB) lo scorso 28 febbraio a Napoli, presso la Biblioteca Fra Landolfo Caracciolo, nel complesso monumentale di San Lorenzo Maggiore.

Quello della valorizzazione può sembrare un tema poco rilevante per una associazione come AICRAB, il cui obiettivo strategico è la promozione di attività e interventi di salvaguardia del patrimonio custodito in biblioteche e archivi.

In effetti, l'iniziativa Oltre le mostre è nata da una lunga riflessione del Consiglio Direttivo dell'Associazione e, in definitiva, dalla consapevolezza che questo patrimonio soffre ancora di una oggettiva condizione di marginalità nel generale contesto dei beni culturali anche perché è poco conosciuto dal comune cittadino, che di norma ha più familiarità con i capolavori dell'arte e dell'archeologia che con le pergamene e le carte antiche e moderne. Il fatto di ripensare alle modalità della sua promozione presso il largo pubblico ci è apparsa un'esigenza non più procrastinabile se desideriamo che libri e documenti comincino a godere di considerazione e importanza pari a quelle attribuite ad altre testimonianze materiali tutelate dalla nostra legislazione.

Anche la scelta della sede, Napoli, non è stata casuale; pertanto, prima di entrare nel merito dei contenuti del seminario, mi sembra opportuno premettere alcune considerazioni generali utili a chiarire questi orientamenti di tema e di luogo. 
All'Associazione AICRAB sono iscritti archivisti, bibliotecari e restauratori ${ }^{1}$ che ne hanno compreso e ne condividono lo spirito e le finalità, vale a dire mettere in rapporto tra di loro le diverse competenze relative alla conservazione del patrimonio culturale. A lungo questi professionisti sono stati condizionati da percorsi di formazione assai eterogenei, incontrando serie difficoltà di dialogo, hanno stentato a condividere le esperienze, nonostante l'interesse comune - la salvaguardia dei materiali archivistici e bibliografici - e la convenienza a collaborare gli uni con gli altri. In particolare, i restauratori hanno scontato il fatto che la loro professionalità fosse tradizionalmente vincolata a una trasmissione delle conoscenze di tipo artigianale, finalizzata all'apprendimento del 'mestiere' in laboratori già avviati o in corsi di formazione professionale; irrilevante la frequentazione di università e la familiarità con le discipline storiche, che invece caratterizzano i percorsi di studio di archivisti e bibliotecari, a loro volta semmai carenti di formazione specifica e pratica sui temi della conservazione.

Questo stato di cose ha determinato fino almeno agli anni Ottanta del secolo XX una prassi 'disarticolata' della conservazione. Da archivisti e bibliotecari essa è stata intesa principalmente come conoscenza e trasmissione della componente testuale di libri e documenti (concetto dal quale deriva, fuorviante ma dura a morire, la convinzione che digitalizzare i testi equivalga a conservare gli originali), con una conseguente minore considerazione per le loro componenti materiali. La risoluzione dei problemi di conservazione è stata demandata ai restauratori, che hanno avuto la tendenza a concentrarsi sugli aspetti tecnici del loro intervento, indirizzato a garantire una ripresa di funzionalità dell'oggetto libro/documento attraverso la sostituzione delle parti più logorate (a cominciare dalle legature), piuttosto che ad assicurare il consolidamento degli elementi e delle strutture originali.

Alcuni importanti cambiamenti sono però intervenuti negli ultimi decenni.

Siamo debitori all'archeologia del libro per aver evidenziato come la conoscenza delle tecniche e dei materiali utilizzati nella manifattura del libro e del documento sia essenziale, accanto alla conoscenza dei testi, per ricostruire l'ambiente culturale che li ha prodotti. Sulla base di questa consapevolezza, il restauro si è andato orientando sempre più decisamente verso modalità operative meno invasive.

Abbiamo anche compreso come i risultati ottenuti dall'intervento di restauro rischino di essere vanificati se i manufatti restaurati ri-

1 L'Associazione è rivolta anche ad altri professionisti impegnati nella conservazione del patrimonio archivistico e librario e agli studenti che stanno affrontando questi aspetti, nonché ai sostenitori a vario titolo delle sue attività. Per conoscere AICRAB: https://www.aicrab.org. 
entrano in ambienti inadeguati alla loro conservazione nel lungo periodo. A questo proposito, dobbiamo al Codice dei beni culturali e del paesaggio (Decreto Legislativo 22 gennaio 2004, n. 42, d'ora in poi Codice) il merito di aver posto l'accento sulla necessità che la conservazione si sviluppi in diverse fasi progressive, tra di loro coerenti e coordinate, e l'aver individuato le figure professionali che possono offrire un contributo in questo ambito.

Secondo il Codice, infatti, la conservazione si articola in studio, prevenzione, manutenzione e restauro (art. 29) e per l'articolo 1 della successiva legge 22 luglio 2014, n. 110 queste attività e interventi sono

affidati alla responsabilità e all'attuazione, secondo le rispettive competenze, di archeologi, archivisti, bibliotecari, demoetnoantropologi, antropologi fisici, restauratori di beni culturali e collaboratori di beni culturali, esperti di diagnostica e di scienze e tecnologia applicate ai beni culturali e storici dell'arte, in possesso di adeguata formazione ed esperienza professionale.

Ne discende che la conservazione del patrimonio archivistico e bibliografico deve essere un impegno condiviso verso il conseguimento di un obiettivo comune: la fruizione di questo patrimonio nel presente e la sua trasmissione al futuro, nel rispetto delle peculiarità testuali e fisiche che lo caratterizzano in quanto prodotto della società umana in un dato luogo e in una data epoca, in altre parole come testimonianza storica.

Negli ultimi anni, ancora per disposizione del Codice e dei successivi decreti legislativi, la formazione dei restauratori ha assunto un'impostazione universitaria ${ }^{2}$ che prevede l'acquisizione della laurea magistrale al termine di un corso quinquennale a ciclo unico. Il curriculum comprende discipline di carattere sia storico che scientifico oltre che attività di progettazione e realizzazione di interventi diretti sul patrimonio.

Si tratta di una evoluzione culturale, presupposto fondamentale per un dialogo più consapevole tra restauratore e bibliotecario conservatore $^{3}$ e tra restauratore e archivista.

2 D.M. 26 maggio 2009, n. 87. «Regolamento concernente la definizione dei criteri e livelli di qualità cui si adegua l'insegnamento del restauro, nonché delle modalità di accreditamento, dei requisiti minimi organizzativi e di funzionamento dei soggetti che impartiscono tale insegnamento, delle modalità della vigilanza sullo svolgimento delle attività didattiche e dell'esame finale, del titolo accademico rilasciato a seguito del superamento di detto esame ai sensi dell'articolo 29, comma 8 e 9, del Codice dei beni culturali e del paesaggio».

3 Mi riferisco ai bibliotecari responsabili del patrimonio nelle biblioteche di conservazione, che non coincidono con la totalità delle biblioteche italiane, la grande maggio- 
Va infine sottolineato il fatto che, per quanto la genesi e la mission di biblioteche e archivi possano divergere, la gestione della conservazione riguarda i materiali (carte, pergamene, inchiostri, cuoi, tele...) e le strutture del bene archivistico/librario e necessita delle medesime competenze e metodologie indipendentemente dal fatto che libri e documenti (o le foto, i manifesti e altro) si trovino in una biblioteca ovvero in un archivio.

In questo contesto, AICRAB si è proposta come punto di incontro tra le diverse professionalità impegnate nella salvaguardia del patrimonio, contribuendo alla loro formazione e aggiornamento attraverso l'organizzazione di convegni, seminari e giornate di studio nelle quali ha coinvolto di volta in volta istituzioni e università. ${ }^{4}$

Se la conservazione è il tema fondamentale di AICRAB, la promozione della conoscenza costituisce comunque un obiettivo importante, perché, come anticipato, appena una ristretta aliquota di cittadini accede alle raccolte che ha comunque l'onere economico di mantenere e tutelare sostenendone il costo pubblico.

Come in diverse occasioni ho già sottolineato, il patrimonio del quale non si ha contezza è un patrimonio che tende a 'sfilarsi' dall'interesse comune, al quale si decide prima o poi di non destinare più le risorse umane ed economiche indispensabili per consentirne la gestione e garantire lo svolgimento delle attività fondamentali di studio, di prevenzione dei rischi, di manutenzione e di restauro, cioè la sua conservazione. Mi sembra dunque importante il fatto che il Codice individui in entrambe le azioni di conservazione e di valorizzazione il presupposto per garantire una cura costante dei beni culturali e la loro salvaguardia nel presente e per il futuro.

Va considerato che la fruizione dei materiali librari e archivistici risente di una peculiare contraddizione che non condivide con gli oggetti del patrimonio artistico o archeologico: la fruizione di libri e documenti è infatti vincolata alla consultazione e di conseguenza alla sollecitazione delle loro componenti materiali, richiede consapevolezza e di norma ne influenza la conservazione.

Anche per questo, sempre di più la valorizzazione è coincisa quasi a senso unico con l'organizzazione di mostre, che confinano il libro

ranza delle quali annovera tra i suoi compiti fondamentali la diffusione della conoscenza, connotandosi come biblioteche pubbliche di informazione. Pur tuttavia non è infrequente che biblioteche pubbliche di informazione possiedano fondi più o meno cospicui di libri riconosciuti come beni culturali, che devono essere conservati. Se in un'epoca di gravi carenze negli organici può parere eccessivo chiedere la presenza di un bibliotecario formato per la conservazione, sarebbe forse opportuno che alla soluzione degli eventuali problemi in tale ambito collaborassero consulenti esterni qualificati.

4 Per le attività organizzate da AICRAB in questi anni si rinvia al sito dell'Associazione e in particolare all'indirizzo: https://www.aicrab.org/eventi/elenco/?tribe_ paged=1\&tribe_event_display=list\&tribe-bar-date=2013-10-30\&order=DESC. 
e il documento 'sotto vetro', assimilandoli alle opere d'arte piuttosto che evidenziarne la specificità: sicché manufatti dinamicamente tridimensionali vengono ridotti a oggetti staticamente piani.

Mette conto notare inoltre che, nella gran parte delle mostre che espongono beni storico-artistici, archeologici ma anche di storia naturale e della scienza, libri e documenti svolgono di norma un ruolo ancillare quasi a legittimare, con la testimonianza scritta, la rilevanza storica degli altri materiali presenti, veri protagonisti delle esposizioni. Infine, si deve ammettere che le mostre di soli libri e documenti suscitano di norma uno scarso interesse nel largo pubblico, a meno che essi siano dotati di un appariscente corredo iconografico e si prestino ad essere assimilati alle opere d'arte.

Come promuoverne la conoscenza ben oltre l'ambito degli studiosi e degli specialisti e quali sono le figure professionali che, in un contesto interdisciplinare, possono contribuire a costruire dei percorsi di avvicinamento agli aspetti testuali e materiali di questo patrimonio? Questi gli spunti di riflessione offerti dal seminario napoletano, che si è articolato in una mattinata di relazioni su esperienze concrete di valorizzazione e in una tavola rotonda pomeridiana nella quale si sono ripresi i temi proposti, allargando il dibattito al pubblico in sala.

Qualche considerazione sul luogo. Fino al febbraio scorso gli eventi AICRAB si erano concentrati sostanzialmente nel Centro-Nord della penisola, con l'eccezione delle attività organizzate qualche tempo prima a Siracusa e a Palermo dalla sezione siciliana dell'Associazione coordinata da Claudia Giordano.

La preferenza per Napoli e in particolare per la Biblioteca Fra Landolfo Caracciolo si è determinata grazie al fattivo rapporto di collaborazione con l'Associazione San Bonaventura Onlus, costituitasi nel 2014 - significativamente, poco dopo AICRAB, nata nel 2013 - e impegnata soprattutto nel Centro-Sud in progetti di tutela e valorizzazione che insistono sul patrimonio artistico, museale e librario, favorendo ugualmente il dialogo interprofessionale.

All'Associazione San Bonaventura, che si è adoperata per rendere possibile lo svolgimento di questo incontro nonostante le recenti restrizioni imposte dall'emergenza Covid-19, vanno naturalmente i sensi della mia riconoscenza. Non posso trascurare l'impegno del Consiglio Direttivo AICRAB e delle Edizioni Ca' Foscari che hanno voluto ospitare questa pubblicazione nella collana «Studi di archivistica, bibliografia, paleografia» diretta dalle professoresse Flavia De Rubeis e Dorit Raines, che ringrazio sentitamente. Con Flavia De Rubeis ho un personale debito di gratitudine per aver voluto direttamente contribuire alle spese di pubblicazione del volume.

In qualità di Presidente AICRAB mi è parso doveroso far precedere $\mathrm{i}$ testi di questa pubblicazione da una breve introduzione soprattutto per esprimere stima e riconoscenza alle amiche e agli amici che, 
nonostante le difficoltà di questo periodo, hanno confermato il loro impegno e consentito con la loro presenza lo svolgimento del seminario e la realizzazione degli Atti.

Anna Busa, esperta di marketing e di strategie digitali, apre con il primo contributo e presenta i meccanismi che influenzano i nostri rapporti con il mondo digitale, fornendo un quadro dei numeri e degli orientamenti dei navigatori del web e ponendo l'accento sulle modificazioni che gli strumenti informatici hanno indotto nella vita quotidiana, a partire dagli ambienti commerciali fino a raggiungere i contesti culturali nei quali si propone il 'marketing umanistico'.

Nella seconda parte della relazione, Busa evidenzia le tecniche utili a valorizzare il ruolo svolto da queste istituzioni nel rapporto con un'utenza sempre più attiva nell'uso e nell'applicazione degli strumenti digitali agli ambiti della cultura.

Muovendo i passi proprio dalle profonde modificazioni indotte nelle biblioteche e negli archivi dalla multimedialità, Michela Corsini ci coinvolge nelle modalità di valorizzazione dei luoghi della memoria, che ella ben conosce in qualità di responsabile sia della Biblioteca che dell'Archivio storico del Comune di Seravezza (Lucca). Le due istituzioni sono riunite sotto il medesimo tetto, ma le loro peculiarità sono diversamente caratterizzate anche attraverso specifiche scelte di comunicazione.

Nella Biblioteca è corrente l'impiego dei social networks, in particolare Facebook e Instagram che dall'iniziale, basilare utilizzo come sorta di bacheca degli avvisi, si sono gradualmente trasformati in strumenti di promozione delle iniziative e delle attività bibliotecarie. Un ulteriore mezzo di coinvolgimento di fasce sempre più ampie di popolazione, segnatamente di quella giovanile, è lo svolgimento di progetti di tirocinio e, in particolare, le opportunità offerte dai progetti Alternanza Scuola Lavoro, innovazione introdotta qualche anno fa dalla legge Buona Scuola, spesso controversa ma che, ben indirizzata, ha saputo dare qui risultati apprezzabili.

La valorizzazione dell'Archivio storico di Seravezza si è articolata secondo modalità diverse: oltre all'esposizione di materiale restaurato e alle visite guidate per gli studenti degli istituti presenti sul territorio, sono stati realizzati laboratori didattici sia per l'infanzia (Filomena la Pergamena è il personaggio creato per spiegare ai più piccoli la struttura di un archivio), sia per i più grandi, correlando le raccolte documentarie con la storia locale, e coinvolgendo i cittadini in un percorso di riappropriazione dell'archivio.

Ci spostiamo con Paola Errani dalla biblioteca pubblica di informazione a una delle nostre biblioteche storiche più belle, la Biblioteca Malatestiana di Cesena, presso la quale Errani a lungo ha prestato la propria opera come responsabile della conservazione. Il suo contributo sintetizza con grande efficacia il senso e le possibilità di valorizzazione di una biblioteca monumentale e del suo 
patrimonio. L'impresa di rendere la Biblioteca Malatestiana fruibile al pubblico più largo potrebbe apparire facile: le sue caratteristiche storiche e strutturali ne fanno un gioiello biblioteconomico giacché non esiste al mondo biblioteca più antica che abbia conservato l'architettura, il mobilio, i codici così come li volle il signore di Cesena, Malatesta Novello, a metà del secolo XV. Questo contesto tuttavia implica di per sé rilevanti responsabilità poiché l'elevato numero di visitatori si traduce in un pubblico assai composito, che inevitabilmente si rapporta a una istituzione come la Malatestiana in modo singolare. Il quadro definito da Errani considera infatti le più diverse esigenze di fruizione, pur senza dimenticare la priorità della salvaguardia. Rinviando alla diretta lettura del suo contributo, voglio comunque sottolineare l'articolazione dell'offerta destinata agli studenti. Agli alunni della scuola primaria si insegna a 'legare' un piccolo libro, agli adolescenti della scuola secondaria di primo grado si propone l'esercizio delle antiche scritture gotica e umanistica ripercorrendo i passi di Jean d'Epinal, lo scriba francese che esemplò alcune decine di codici malatestiani; più complesso il percorso per i giovani delle scuole secondarie di secondo grado, ai quali viene illustrata la storia parallela che nel terzo quarto del secolo XVI si svolgeva a Cesena e a Magonza, dove Gutenberg realizzava le prime prove di stampa a caratteri mobili. Ai ragazzi è offerta sia l'esperienza degli amanuensi, con calami e penne d'oca, che quella tipografica, che per mezzo di un piccolo torchio consente loro di imprimere un proprio ex libris.

Anna Manfron, già responsabile dell'Istituzione Biblioteche del Comune di Bologna e della Biblioteca dell'Archiginnasio, apre la sua relazione citando il Manifesto IFLA/UNESCO che individua nelle biblioteche pubbliche le 'vie di accesso alla conoscenza'. Questo principio consente di stabilire un rapporto diretto con la Carta costituzionale italiana, garante non soltanto della salvaguardia del patrimonio culturale ma anche della produzione e della redistribuzione di conoscenza fondamentale per il consapevole e pieno sviluppo degli individui. La relatrice concentra l'attenzione sui fondi d'autore presenti nelle biblioteche e sull'importanza della loro valorizzazione, che potrà realizzarsi compiutamente soltanto al termine di un processo che comprenda la conservazione e l'attività catalografica e descrittiva. I materiali eterogenei che confluiscono in questi fondi (carte manoscritte, testi a stampa, fotografie, opere d'arte, oggetti, arredi ecc.) comportano competenze professionali assai articolate per la loro corretta gestione. Manfron conclude affrontando il tema delle mostre, che, nel caso specifico dei fondi d'autore, consentono non solo di avvicinare il pubblico all'opera del singolo personaggio ma di richiamare il contesto storico e culturale dal quale l'opera ha tratto origine, assumendo un potere evocativo estremamente più efficace della lettura di qualunque saggio sul tema. 
Le questioni che si pongono Sofia Stefani ed Elisa Di Liberato vertono attorno a un ampio progetto (Archiporto) di catalogazione, conservazione e valorizzazione dell'Archivio da Porto custodito presso il Castello Porto-Colleoni-Thiene (VI), progetto tuttora in corso e che ha l'obiettivo fondamentale di disseminare la conoscenza di questo patrimonio in un bacino di utenza ben più ampio della famiglia alla quale esso appartiene.

Sofia Stefani, archivista, racconta la genesi e lo sviluppo del progetto e sottolinea le potenzialità offerte dalle risorse digitali - a cominciare dalla riproduzione dei catastici fino all'ingresso nel portale archivistico della Biblioteca civica Bertoliana di Vicenza - che consentono di mettere a disposizione del più largo numero di studiosi i risultati di questo lavoro.

Elisa Di Liberato, sviluppatrice di progetti culturali, si concentra invece sull'aspetto della valorizzazione, che ha puntato in un primo momento a correlare questi archivi con il territorio e successivamente a capire quali fossero gli strumenti più efficaci per sollecitare l'interesse del pubblico verso i documenti originali e i loro contenuti. Ne è nato un gioco di carte animate da personaggi e storie affiorate dallo studio diretto dei documenti, gioco nel quale non mancano riferimenti al mondo contemporaneo, come a tracciare un'ideale continuità tra vicende del passato e storia presente.

La terribile alluvione del 1966, causa di danni incalcolabili al patrimonio culturale fiorentino, è al centro dell'iniziativa della Biblioteca Nazionale Centrale di Firenze (BNCF) presentata da Alessandro Sidoti, responsabile del settore conservazione e restauro della BNCF. Il progetto, denominato Safari della memoria, ha avuto appunto l'obiettivo di andare 'oltre le mostre' per offrire, in occasione del cinquantennale, una ricostruzione delle fasi principali dell'alluvione e dei primi soccorsi. Rielaborando le riprese fotografiche originali del novembre 1966, è stato così strutturato un percorso virtuale parallelo alla visita (in presenza e silenziosa) della BNCF: con un visore o un tablet tra le mani, i visitatori potevano scorrere le immagini storiche e confrontarle con l'aspetto odierno dei medesimi ambienti della biblioteca. Accanto a questa, sono state messe a punto altre iniziative, tra cui l'applicazione Arno66-Time Travel, un viaggio nel tempo che consentiva di passeggiare nel capoluogo toscano per riconoscere i luoghi principali dell'alluvione. Infine, va ricordata l'attività di digitalizzazione delle oltre 3.500 immagini che testimoniano l'evento del 1966, oggi disponibili nell'archivio fotografico digitale sul sito della Biblioteca nazionale fiorentina.

Anche la proposta realizzata dall'Associazione San Bonaventura assume un carattere innovativo nel progetto San Lorenzo Escape, introdotto da Daniele Ferraiuolo: la conoscenza della storia napoletana, dei documenti e delle collezioni librarie della Biblioteca è alla base del progetto, per il quale l'Associazione ha attinto alle esperien- 
ze maturate nell'applicazione delle tecniche di narrazione basate su videoproiezioni digitali interattive. Il gioco San Lorenzo Escape che ne è scaturito prevede il passaggio dei giocatori (gruppi di visitatori) in quattro diversi ambienti ricostruiti in biblioteca, che costituiscono altrettante tappe di un 'percorso di fuga' attraverso il quale occorre sciogliere gli enigmi di carattere storico archivistico-bibliografico proposti utilizzando strumenti multimediali di notevole effetto, come può testimoniare chi scrive, che si è voluta cimentare - con alterna fortuna - in questa esperienza immersiva e coinvolgente. Sono particolarmente lieta del fatto che, anche grazie al San Lorenzo Escape, si sia notevolmente incrementato il rapporto con l'utenza poiché il gioco ha indotto a entrare in biblioteca una parte di cittadini che non l'aveva ancora frequentata.

Prima tra gli ospiti della tavola rotonda del pomeriggio, Mariella Guercio, docente di archivistica e già presidente dell'Associazione Nazionale Archivistica Italiana (ANAI), esprime nel suo testo un fermo scetticismo nei confronti delle mostre in generale e, segnatamente, dell'applicazione del 'mostrismo' (neologismo che ella riprende con una citazione dell'autore, Armando Petrucci) in ambito documentario. Guercio sottolinea come gli archivisti abbiano costantemente rigettato l'adesione acritica a iniziative - assai diffuse dagli anni Novanta del secolo scorso - risoltesi sovente in un investimento di risorse economiche e umane che avrebbero potuto avere una migliore destinazione se rivolte al funzionamento degli archivi. Viene altresì evidenziata la carente attività di valorizzazione del patrimonio documentario del nostro Paese, che ha condotto a una sorta di «rimozione collettiva o di amnesia generalizzata» (106) dell'esistenza di tale patrimonio. A questo si aggiungono il depauperamento di risorse umane, professionali ed economiche che ha colpito negli ultimi anni le cenerentole della cultura, vale a dire gli archivi e le biblioteche, nonché le debolezze in tema di aggiornamento professionale e di formazione sia nell'ambito delle associazioni di settore sia in quello accademico, carenze che determinano il rischio per questo settore di finire abbandonato a se stesso. Non manca un accenno alla digitalizzazione, considerata, per molti anni, la soluzione ideale per i problemi degli archivi: se non c'è dubbio che essa consente di mettere a disposizione degli studiosi importanti raccolte consultabili anche da postazioni remote, per contro la conservazione del materiale digitale rappresenta un dilemma del quale è difficile venire a capo.

Il direttore della Fondazione Ugo e Olga Levi Onlus di Venezia, Giorgio Busetto, richiama il problema cruciale della selezione nella conservazione, ribadendo la necessità di scegliere il materiale da salvaguardare giacché non è realisticamente possibile conservare tutto.

Comprendo queste riflessioni, che mi danno l'opportunità di ricordare come i documenti siano soggetti a una selezione a monte che 
scarta tutto il materiale non riconosciuto di rilevanza storica tale da determinarne l'obbligo di essere trasmesso ai posteri. Solo ciò che rimane in archivio entra a far parte del patrimonio culturale proprio perché venga conservato (art. 10 del Codice).

Diverso è il discorso per il materiale librario. Non tutti i libri sono beni culturali, anzi: per la gran parte di essi, quelli che si trovano nelle biblioteche pubbliche d'informazione (quali le biblioteche scolastiche ricordate dall'autore) il valore più rilevante è quello di uso e, quando logorato dall'uso, il volume può essere riparato o sostituito con un'altra copia.

Anche per quanto riguarda la produzione editoriale contemporanea, la conservazione è più che selezionata, riguarda pochi esemplari (tre o quattro, a seconda della regione nella quale ha sede l'editore) ed è regolata dalla normativa del deposito legale.

Tra le tante sollecitazioni che propone, Busetto ricorda l'importanza di consentire l'accesso del pubblico al patrimonio, giacché è più immediato il controllo delle condizioni di conservazione del materiale 'frequentato' piuttosto che di quello custodito in depositi o magazzini.

Questo pone l'accento sulla ricaduta che la digitalizzazione comporta in termini di fruizione degli originali: essa favorisce la frequentazione di biblioteche e archivi o, viceversa, porta verso il progressivo svuotamento delle sale di consultazione?

Di tutt'altro tenore è la relazione di Micaela Procaccia, presidente dell'Associazione Nazionale Archivistica Italiana, ANAI, che riprende e amplia il tema del 'mostrismo' di Armando Petrucci (termine definito «come una variante di approccio superficiale al patrimonio culturale»), esprimendo disappunto per le modalità con le quali vengono di norma esposti i documenti d'archivio, offerti alla lettura ma senza alcun contesto che ne evidenzi le peculiarità e ne favorisca la comprensione anche a coloro che non hanno familiarità con le scritture documentarie.

Da ciò l'autrice fa discendere la constatazione che il patrimonio archivistico è destinato allo studio piuttosto che all'esposizione, anche se è possibile formulare alcune proposte per la sua migliore valorizzazione, coinvolgendo magari le più giovani generazioni di potenziali utenti. Ciò è avvenuto, ad esempio, nel caso degli Archivi di Stato che hanno organizzato una caccia al tesoro per bambini delle scuole primarie utilizzando i fac-simili dei documenti originali. Procaccia non dimentica infine il contributo che può venire, in questo periodo nel quale la grave situazione sanitaria limita i movimenti, dalle raccolte archivistiche che le tecniche di digitalizzazione mettono ormai a disposizione di un largo pubblico. L'augurio chiaramente espresso è quello che in un futuro che confidiamo prossimo, post-pandemico, si investa davvero «nella tutela del patrimonio, nel reclutamento dei professionisti, nella progettazione della comunicazione, ricordando il ruolo strategico degli archivi nella costruzione di quel sentimento 
civile di appartenenza alla comunità che oggi sta tenendo insieme la società italiana» (125).

Nel suo contributo, Martín M. Morales, direttore dell'Archivio della Pontificia Università Gregoriana, si chiede (ci chiede?) se prima di andare 'oltre le mostre' se ne sia compresa appieno la problematica giacché, citando la Metafisica aristotelica, difficilmente potrà sciogliere un nodo chi non sa come farlo. Entrando nel cuore del problema, Morales sottolinea come le mostre di materiali archivistici e librari pongano a repentaglio la conservazione di questo patrimonio. Pur tuttavia, esse continuano a diffondersi più per la domanda posta dal sistema economico - che assimila i beni culturali al 'petrolio dell'Italia' o, poco più elegantemente, al 'cibo per la mente' - piuttosto che per una reale esigenza culturale.

L'altra connotazione che Morales intravede nel libro e nel documento oggetto di mostre è quella di 'cosa semiofora' difesa da alcuni e bersagliata da altri, connotazione che contrasta comunque con la semantica di mercato basata piuttosto sul binomio scarsità/abbondanza donde origina l'inclusione, quantomeno per il libro antico, nell'ambito del patrimonio culturale. D'altra parte, anche il termine 'valorizzazione' potrebbe etimologicamente essere letto come l'incremento (fittizio, secondo gli autori citati) del valore di una 'merce'. Mi è impossibile rendere in modo sintetico la piena articolazione dei temi affrontati da Morales, dalla digitalizzazione/volgarizzazione, all'abuso di sapere' degli esperti, ma non vorrei omettere di citare la realizzazione, da parte dell'Archivio della Pontificia Università Gregoriana, della piattaforma GATE (Gregorian Archives Text Editing), un ambiente virtuale nel quale i 'saperi' cooperano in modo transdisciplinare «integrando cioè diversi punti di vista nell'osservazione di complessità superiori alle stesse discipline» (134).

Non mi resta che invitare alla lettura diretta di questa e delle precedenti relazioni tutti coloro che avranno avuto la pazienza di seguire i miei argomenti fino a questo punto.

Concludo con una nota personale legata alla situazione che tutti noi stiamo vivendo. L'organizzazione del seminario napoletano è coincisa con l'inizio della pandemia Covid-19 che dalla scorsa primavera ha mutato profondamente le nostre abitudini, i nostri progetti e le nostre aspettative. Alla fine di febbraio 2020, all'approssimarsi del giorno fissato le idee non erano ancora chiare sugli sviluppi che avrebbe avuto la diffusione del virus. Il Consiglio Direttivo AICRAB discusse a lungo se confermare l'incontro e, alla fine, la decisione fu unanimemente positiva. Si trattò di un evento in presenza - era ancora imprevedibile l'affollarsi di impegni su Zoom, Teams, Google Meet e le altre piattaforme che da lì a poco avremmo dovuto gestire - e provvidenzialmente tutto andò nel migliore dei modi, senza conseguenze per la salute degli intervenuti. 
Nei mesi successivi AICRAB ha organizzato alcuni webinar, il primo dedicato alle modalità della ripresa dopo l'emergenza pandemica, il secondo alla risoluzione dei problemi che la chiusura repentina imposta dal confinamento aveva determinato negli archivi e nelle biblioteche, con particolare riferimento all'insorgere di infezioni e infestazioni correlate alla mancanza di controllo dei parametri ambientali nei locali (anche magazzini e depositi librari) rimasti senza personale per alcuni mesi. La numerosa partecipazione a questi seminari online ci ha convinto dell'opportunità di mettere a disposizione le registrazioni dei webinar sul canale YouTube appositamente aperto dall'Associazione. ${ }^{5}$

É, questo, il piccolo contributo di AICRAB a tutti gli archivisti, bibliotecari e restauratori in difficoltà per carenza di mezzi e di linee guida univoche alle quali fare riferimento in un momento particolarmente complesso.

Non c'è forse bisogno di sottolineare come la pratica delle attività di conservazione, sovente negletta già in periodi di normalità, abbia risentito in maniera estremamente negativa dei condizionamenti imposti dal lungo confinamento.

Il fatto che, nei mesi di massima privazione delle nostre possibilità di movimento, le biblioteche di tutto il mondo abbiano messo a disposizione ad accesso libero migliaia di testi in formato digitale non ha protetto i volumi che contenevano quei testi dall'umidità, dalle infezioni, dagli attacchi entomologici. Se ci fosse stato ancora bisogno di comprendere che solo la conservazione è in grado di farsi carico della salvaguardia materiale di libri e documenti, in questo drammatico frangente ne abbiamo fatto l'esperienza peggiore.

Mentre consegno queste note all'editore (ottobre 2020), prima ancora che tutte le biblioteche e gli archivi siano riusciti a riprendere le attività congelate la scorsa primavera e a risolvere le criticità subentrate, la situazione di diffusione del Coronavirus pare precipitare di nuovo e non è possibile prevedere quali saranno i suoi sviluppi futuri. Voglio credere fermamente che non ci ritroveremo in una situazione di crisi sanitaria tale da giustificare una nuova chiusura totale degli istituti, ma ancora più auguro a tutti noi di aver imparato dall'esperienza già subita che il patrimonio non si conserva da solo e che sono necessarie consapevolezza e competenza anche per chiudere le porte di archivi e biblioteche avendo preso tutte le precauzioni preventive affinché non siano ancora sempre i beni culturali a pagare conseguenze insostenibili.

5 L'accesso alle registrazioni dei webinar «Coronavirus: la gestione del materiale archivistico e librario nella fase 2» e «Gli ospiti indesiderati. Fase 2: il rientro negli archivi e nelle biblioteche e il rischio delle infestazioni» è possibile da https:/www.aicrab.org/news/. 


\title{
Patrimonio archivistico e librario e nuove strategie di marketing (digitale) Come ripensare la comunicazione
}

Anna Busa

Marketing \& Digital strategist freelance; Univerisità di Bologna, Italia

\begin{abstract}
The needs and behaviors of each of us are today strongly influenced by the new digital habits. For the library and the archive, understanding its language, expectations and needs is indispensable nowadays to rethink ways, tone of voice and contents of communication.
\end{abstract}

Keywords Marketing. Digital strategy. Library. Archive. Social. Audience development. Audience engagement.

Esigenze e comportamenti di ciascuno di noi sono oggi fortemente influenzati dalle nuove abitudini digitali. Conoscere la diffusione delle piattaforme, comprenderne l'utilizzo da parte delle persone che usano la rete è oggi una condizione indispensabile, per tutti gli ambiti: da quello privato a quello istituzionale.

We are Social ${ }^{1}$ è una socially-led creative agency che da nove anni, in collaborazione con Hootsuite, ${ }^{2}$ azienda che propone piattaforme software specializzate nella gestione dei social media e dei servizi correlati, realizza un report internazionale nel quale ci vengono offerti i macro-scenari della rete:

1 https://wearesocial.com/it/.

2 https://hootsuite.com/it/. 
internazionale e italiano. Dati interessanti che ci aiutano a comprendere diffusione e dinamiche del web.

In Digital2020, ${ }^{3}$ questo è il nome della ricerca di quest'anno, i risultati mostrano con chiarezza la continua espansione della rete e le sue modalità di utilizzo. Su una popolazione mondiale totale di 7,75 miliardi, il $67 \%$ delle persone utilizza i cellulari e il 59\% sono utenti della rete. Gli utenti medi attivi sui social sono 3,8 miliardi con una penetrazione del $46 \%$ e gli «unique mobile phone users» sono 5,19 miliardi pari al $67 \%$ della popolazione.

I dati registrano negli anni una crescita costante: rispetto al 2019, ad esempio, c'è un aumento del 9,2\% degli utenti attivi sui social e sono circa 300 milioni (+7\%) le persone entrate in rete per la prima volta nel 2019.

Anche la nostra 'giornata tipo' è cambiata. Il report evidenzia che, mediamente, passiamo oltre 6 ore e mezzo collegati alla rete e di questo tempo circa 2 ore e mezzo sono impiegate sui social.

Molto interessante è l'analisi di cosa facciamo in rete e, in particolare, quali sono i siti maggiormente utilizzati. Al primo posto troviamo Google, quindi facciamo ricerche (informazioni, servizi, notizie, ecc.). Poi guardiamo video (infatti al secondo posto c'è YouTube) e stiamo sui social (al terzo posto troviamo Facebook). Nella top ten dei siti più visitati si trovano altri motori come Baidu (il principale motore di ricerca in lingua cinese, al quarto posto), Yahoo! (in ottava posizione) e Yandex (il search engine in russo al decimo posto). Completano l'area social Twitter e Instagram, rispettivamente al sesto e settimo posto. Da segnalare Wikipedia, al quinto posto.

L'Italia conta 60 milioni di abitanti e 80 milioni di cellulari (il 133\%); l'82\% della popolazione italiana è utente della rete e il 58\% delle persone usa i social. ${ }^{4}$

Verrebbe da dire che siamo veramente un popolo di navigatori (del web).

Per quanto riguarda l'uso e la diffusione dei social, così come nel 2019, in Italia YouTube è al primo posto, WhatsApp al secondo, Fa1 cebook al terzo. Instagram è al quarto posto con una crescita dal $55 \%$ al $64 \%$.

Questi dati ci testimoniano quanto sappiamo già: siamo tutti connessi, sempre online. È la trasformazione digitale. Un fenomeno che investe diversi aspetti della società: dal tessuto produttivo ai servizi, dalle imprese alle istituzioni, alla vita di ciascuno di noi.

I diversi ambiti sociali hanno proprie declinazioni di questo fenomeno, ma in maniera liquida sono coinvolti aspetti comuni, a pre-

3 https://wearesocial-net.s3-eu-west-1.amazonaws.com/wp-content/uploads/ common/reports/digital-2020/digital-2020-global.pdf.

4 https://wearesocial.com/it/digital-2020-italia. 
scindere dalla natura e dalla missione dell'organizzazione con cui interagiamo. Ad esempio, noi usiamo lo stesso approccio mentale e ci aspettiamo che le app del nostro cellulare si comportino nello stesso modo nel momento in cui paghiamo un parcheggio, richiediamo l'estratto conto tramite l'home banking o scarichiamo un e-book dalla biblioteca con il digital lending. Sono le cosiddette 'aspettative liquide', ${ }^{5}$ che mi portano ad agire in modo simile indipendentemente dal contesto in cui mi trovo. In altre parole, vivo una «user experience» trasversale.

E evidente come siamo di fronte a un insieme di modificazioni tecniche, certamente, ma soprattutto culturali e organizzative che riguardano persone, processi e tecnologie. L'azione combinata di questi elementi permette di erogare nuovi servizi e creare nuove modalità di interazione. È un cambiamento che ci porta a riconsiderare situazioni, scenari, abitudini. La conseguenza di questa vera e propria rivoluzione è anche una mentalità diversa. Mentalità che si traduce in una maggiore attenzione nel comprendere i bisogni di tutti noi, nella consapevolezza che sono gli utenti a governare il processo, cercando le informazioni in rete, muovendosi in autonomia e quindi, alla fine, facendo la propria scelta. Per le imprese questo si traduce in una irrinunciabile modifica strutturale anche dei loro processi produttivi.

E fuori dagli ambiti commerciali? È opportuno riflettere sulla possibilità di introdurre e usare nuovi strumenti di marketing e di comunicazione anche per la cultura. Non dimentichiamo che in rete ci sono pubblici potenzialmente interessati a scoprire o approfondire la conoscenza dei patrimoni e dei servizi di biblioteche e archivi. Vale la pena, quindi, riflettere su come il marketing in generale e quello digitale in particolare potrebbe (o meglio, dovrebbe) fare squadra insieme alle istituzioni culturali.

Nella prefazione di Marketing delle Arti e della Cultura, si evidenzia come il marketing per molto tempo sia stato

percepito come un concetto ambiguo, da maneggiare con le dovute cautele e le opportune profilassi, che evocava a molti operatori del settore spettri e scenari inquietanti (Colbert et al. 2015, 16)

come quello di una possibile commercializzazione della cultura. La necessità di rendere le istituzioni culturali protagoniste della nuova dimensione digitale, le sta aiutando a mutare atteggiamento nei confronti del marketing e a cogliere quanto di utile il web possa offrire

5 «Liquid expectations» by Fijordnet https://www.fjordnet.com/conversations/ liquid-expectations/. 
per migliorare il nostro rapporto con gli utenti acquisiti e potenziali. ${ }^{6}$ Lo stesso marketing è cambiato. Come scrive Philip Kotler:

Il comportamento e i valori delle imprese sono sempre più sotto la lente dell'opinione pubblica. Questo fa sì che i consumatori possano scambiarsi con grande facilità opinioni su imprese, prodotti e marche e discutere delle loro performance funzionali e sociali. La nuova generazione di consumatori è molto più attenta ai problemi e alle preoccupazioni di natura sociale (Kotler, Kartajaya, Setiawan 2010, 236)

Questo porta a privilegiare messaggi pubblicitari che fanno leva sul motivo per cui devi acquistare, sulla sensazione che provi a seguito dell'acquisto e non tanto sul prodotto. Ė il marketing umanistico, che privilegia empatia, coinvolgimento, emozione avendo ben chiaro che le decisioni dei consumatori nascono sempre di più anche dalle loro percezioni.

Consumatori? Nel mondo culturale noi non abbiamo consumatori! Ne siamo proprio sicuri? In fondo potremmo spingerci a definire i nostri utenti, i nostri pubblici «consumatori di cultura». Le biblioteche, gli archivi non hanno prodotti da vendere, non sono supermercati e non hanno l'obiettivo del profitto. Qual è, quindi, il legame fra biblioteca, archivio e marketing umanistico? Quali sono gli elementi che connotano la biblioteca o l'archivio e sono riconducibili ai principi del marketing umanistico? Nel caso della biblioteca certamente l'ascolto, il servizio come risposta ai bisogni, il dialogo, l'informazione, la capacità di instaurare una relazione duratura con i propri utenti, la competenza e la capacità di accoglienza. A tutto questo si aggiunge la presenza di un'identità, quella della biblioteca o dell'archivio, riconosciuta e riconoscibile, che va promossa e diffusa.

Come afferma Kapferer:

Un brand è un desiderio condiviso ed un'idea esclusiva rappresentato in un prodotto, servizio, luogo o esperienza $(1992,10)$

La biblioteca, quindi, è un brand formidabile, perché ogni biblioteca è un'idea esclusiva che raccoglie servizio, luogo, esperienza. In un unicum. E lo stesso vale per l'archivio.

Il maggiore aspetto di discontinuità del nuovo web con il passato è rappresentato dal superamento della funzione di propagazione dei contenuti, tipica dei mass-media, e dal ruolo più attivo dell'utente. Diffusione e promozione si trovano, quindi, a dover superare la fase

6 Interessante sul tema anche quanto scrive Alessandro Bollo in «50 sfumature di pubblico e la sfida dell'audience development» (2014). 
descrittiva e di tipo broadcast per entrare in una più ampia dimensione di proposizione attiva.

Certamente un brand forte è di aiuto, ma non è sufficiente. Occorre, infatti, adottare anche nuovi modelli di comunicazione connotati da un approccio strategico. È proprio la strategia che rappresenta una novità rispetto alla semplice presenza in rete delle realtà istituzionali.

In altre parole, la definizione di una strategia di marketing permette di inquadrare, all'interno di un sistema di riferimento ben definito, strumenti e attività in modo organico e strutturato. Sito web, catalogo, social network fanno riferimento a una linea di comunicazione comune e concorrono, ciascuno con le proprie caratteristiche, al raggiungimento degli obiettivi identificati. Il fine di questo nuovo approccio è migliorare la capacità di ascolto e di dialogo con gli utenti e fornire una visione arricchita e più ampia dei servizi della biblioteca e dell'archivio.

Articolato in fasi ben definite, il processo di marketing parte dall'analisi degli scenari (interno ed esterno) per posizionare l'istituzione nel suo territorio e nella relazione con gli stakeholders e per studiare quanto internamente è stato fatto per realizzare la sua missione. Prosegue con una accurata definizione degli obiettivi e con la scelta del modello di strategia di marketing più opportuna.

Una delle più interessanti è senza dubbio l'inbound, che ha la caratteristica (e il pregio) di trasformare il marketing dell'interruzione - che cattura l'attenzione dell'utente interrompendo la sua attività (è, per intenderci, quello delle pubblicità in televisione, delle telefonate a tutte le ore, dei banner che oscurano i siti, ecc.) - in marketing del coinvolgimento.

L'inbound è connotato da quattro fasi: attirare, convertire, chiudere, deliziare. Un esempio: ho necessità di un certo servizio ma non ho idea di chi possa erogarlo e con quali modalità. Apro il motore di ricerca (ad esempio Google) e digito alcune parole chiave nel mono campo. In questo momento il mio stato è quello di 'estraneo' perché non conosco ancora i siti nei quali entrerò. Lancio la ricerca e analizzo il risultato: un elenco di siti che Google ha trovato attinenti alla mia ricerca. Ed ecco la prima fase: attirare. Guardo l'elenco dei risultati e scelgo il link che mi sembra più vicino a quanto sto cercando. Quel link mi 'attira', entro nel sito che, nel nostro esempio, sarà quello di una biblioteca o di un sistema bibliotecario, e il mio stato cambia da estraneo a visitatore.

Si attiva, a questo punto, la seconda fase che è quella della conversione: da visitatore, se trovo quello che sto cercando, posso diventare un lead (contatto) perché lascio, ad esempio, l'indirizzo e-mail e $\mathrm{mi}$ iscrivo alla newsletter della biblioteca. In questo modo la biblioteca può inviare aggiornamenti informativi periodici, inviti per eventi, informazioni su nuovi servizi, ecc. 
La terza fase è quella della chiusura, che nel settore privato equivale all'acquisto. Per noi, la trasformazione da lead a utente: mi iscrivo alla biblioteca online, vado in biblioteca a iscrivermi, oppure richiedo il primo documento in prestito. In sostanza compio un'azione che rappresenta il mio nuovo stato di utente. Il deliziare è la quarta fase, la più delicata: da utente mi trasformo in fan, parlo della biblioteca ai miei amici, evidenzio i servizi, condivido le iniziative, divento di fatto un promoter (Busa 2019, 14 ss.).

Le fasi dell'inbound accompagnano e facilitano l'azione di incontro con i pubblici in rete, anche grazie a specifici strumenti e attività. Ad esempio, l'applicazione delle regole SEO (Search Engine Optimization) che aiutano a migliorare la scansione, l'indicizzazione e il posizionamento di un'informazione o contenuto presente in un sito web o una pagina, da parte dei crawler dei motori di ricerca (quali ad esempio Google, Yahoo!, Bing, Yandex, Baidu ecc). Al SEO si affiancano, ad esempio, l'introduzione dell'e-mail marketing con l'invio di newsletter periodiche e una presenza attiva, organizzata e non casuale sui social.

Strumenti e attività che devono essere approfonditi e adattati alle realtà istituzionali. Una pagina Facebook o un profilo Instagram, un Opac portalizzato devono essere visti come strumenti di una strategia di marketing definita adeguatamente e senza la quale granularità e frammentazione hanno il sopravvento, indebolendo tutta la comunicazione istituzionale (cf. Roncaglia 2019).

La gestione dei social non andrebbe affidata alla creatività del momento, ma deve seguire un piano editoriale nel quale si definisce quando e cosa postare. Eventi e iniziative, senza trasformare la pagina Facebook in una bacheca di annunci, si devono alternare ad altri contenuti quali, ad esempio, proposte di lettura, incontri con gli autori, ecc. Un ruolo da protagonista deve essere riservato al patrimonio che viene mostrato e condiviso. Nella narrazione è importante ci sia posto (finalmente) anche per il lavoro 'dietro le quinte': pensate a quanto potrebbe essere interessante un'immagine che ritrae una o più fasi di un intervento di conservazione, ad esempio di restauro. Per creare empatia, è da non sottovalutare la pubblicazione di qualche gatto di biblioteca o di archivio, raffigurati vicino a scorci suggestivi delle strutture ospitanti oppure, come ha fatto l'Archivio di Stato di Napoli con la pubblicazione nel suo profilo Facebook di una fotografia che raffigura la pausa a base di torta di mele fatta in casa, offerta dagli allievi della Scuola di Archivistica, Paleografia e Diplomatica. Un modo sorridente e garbato per raccontarsi, sapientemente alternato alle iniziative e agli splendidi materiali archivistici che rendono unico il suo patrimonio.

Un altro aspetto sul quale soffermarsi è il linguaggio utilizzato per comunicare la biblioteca e l'archivio. Spesso il linguaggio adottato è troppo scientifico, l'uso di termini specialistici taglia fuori in- 
teri settori di pubblico. Parlare di banco del reference a cui chiedere informazioni non è comprensibile se non a noi bibliotecari. Occorre anche eliminare l' 'istituzionalese' e lasciare spazio a un linguaggio più naturale. Pensate, ad esempio, alle CTA (Call To Action), i piccoli testi scritti sui bottoni che vi richiedono un'azione: «Hai bisogno di aiuto? Siamo qui per te» è molto più piacevole di un secco «accedi».

Molti sono, quindi, gli elementi che concorrono a una comunicazione adeguata alle caratteristiche dell'istituzione e che favorisce audience development e audience engagement. Ricordo che l'audience development comprende, oltre al concetto di sviluppo, anche quello di eterogeneità dei pubblici della rete e l'audience engagement esprime la capacità di creare le condizioni di un coinvolgimento sempre più empatico dei pubblici stessi.

Eterogeneità e coinvolgimento, due obiettivi fondamentali. Come raggiungerli? Concentrandoci sulle caratteristiche dei nostri pubblici. Superando il concetto generico di target e introducendo le personas.

Le personas, poiché sono strutture comportamentali comuni, obiettivi, desideri, necessità definite e punti di criticità condivisi, estendono il raggio e la natura delle relazioni con i nostri pubblici. Il risultato è una più consapevole centralità degli utenti e la creazione di occasioni di maggior coinvolgimento e partecipazione dei pubblici. A tutto questo si aggiunge che la nuova consapevolezza di bisogni e aspettative, apre a nuovi spazi di creatività e inclusione e incoraggia l'innovazione. (Busa 2020, 64)

\section{Bibliografia}

Bollo, A. (2014). «50 sfumature di pubblico e la sfida dell'audience development». De Biase, F. (a cura di), I pubblici della cultura. Audience development, audience engagement. Milano: FrancoAngeli, 163-80.

Busa, A. (2019). Come fare marketing digitale in biblioteca. Milano: Editrice Bibliografica.

Busa, A. (2020). Come facilitare l'incontro con i pubblici della biblioteca. Milano: Editrice Bibliografica.

Colbert, F. et al. (2015). Marketing delle arti e della cultura [ebook]. Milano: Rizzoli Etas.

Kapferer, J.-N. (1992). Strategic Brand Management. London: Kogan Page.

Kotler, P.; Kartajaya, H.; Setiawan, I. (2010). Marketing 3.0. Dal prodotto al cliente all'anima. Milano: Il Sole 24 ore.

Roncaglia, G. (2019). L'età della frammentazione. Cultura del libro e scuola digitale. Roma-Bari: Editori Laterza. 



\title{
I luoghi della memoria $\mathbf{2 . 0}$ Strategie di promozione social e digital della biblioteca e dell'archivio
}

\section{Michela Corsini}

Biblioteca comunale e Archivio storico comunale, Comune di Seravezza (LU), Italia

\begin{abstract}
Bringing the new generations, the digital natives, to the 'places of memory' such as library and archive is a challenge that professionals face daily and which requires not only an update of the relative services but also an evolution of the strategies used by the librarian and by the archivist to promote and enhance these places and services. This is the experience of Seravezza (LU) made by social networks, 'biblioselfie', 'bibliocalendars', mannequin challenge and the stories of 'Filomena la Pergamena'.
\end{abstract}

Keywords Places of memory. Library. Archives. Social networks. Marketing strategies.

Sommario 1 ll sogno di Borges. - 1.1 Biblioteca e archivio: Giano Bifronte? - 1.2 La biblioteca social: i progetti realizzati presso la comunale di Seravezza. - 2 L'archivio storico comunale tra didattica e digitalizzazione. 
Abbiamo una memoria organica, registrata, custodie ta ed elaborata dal nostro cervello, e sin dalle origini l'uomo si è affidato a una memoria minerale, incidendo pietre o tavolette d'argilla - sino alla memoria di silicio dei nostri computer attuali.

(Umberto Eco, La memoria vegetale e altri scritti di bibliofilia, 2006)

\section{Il sogno di Borges}

La promozione e la valorizzazione dei luoghi della memoria, quali biblioteche e archivi, attraverso i social network e il mondo del web, anche al di fuori da quelle che possono essere definite le 'tradizionali' modalità di organizzazione di eventi culturali, è un'opportunità in più che i professionisti del settore dovrebbero cogliere. Gli archivi, le biblioteche e le professioni ad essi legate, in questi ultimi quindici anni hanno risentito di un profondo cambiamento legato alla multimedialità, al diverso modo di comunicare: tutto è più veloce e immediato. Questo cambiamento non può essere osteggiato, ma preso per mano e gestito, impiegato in maniera corretta e coerente a favore della nostra professione per raggiungere più facilmente gli obiettivi di efficacia ed efficienza nell'ambito della promozione culturale e dei servizi che ne derivano. Ciò non significa che la missione delle biblioteche e degli archivi sia cambiata, le finalità sono le stesse, devono però mutare le modalità di approccio e di promozione di queste realtà, aprendole a nuove possibilità e sviluppando le numerose potenzialità che l'era digitale ci offre. Inevitabilmente è opportuno che anche le nostre professioni di archivista e bibliotecario si adattino al cambiamento, arricchendosi di nuove competenze e aggiornamenti, senza rinnegare nulla del passato ma con uno sguardo puntato al futuro per il raggiungimento della customer satisfaction e la sopravvivenza delle nostre realtà lavorative. Puntare su questo tipo visibilità è uno dei percorsi a mio parere più appropriati per mostrare il contributo che le biblioteche e gli archivi, spesso accusate di non stare al passo coi tempi, possono ancora dare alla società odierna e futura. In questo particolare momento in cui si realizza il sogno di Borges, alla perenne ricerca del 'catalogo dei cataloghi', non possiamo permetterci di essere statici, perché senza movimento non c'è cambiamento e senza cambiamento non c'è evoluzione.

\subsection{Biblioteca e archivio: Giano Bifronte?}

La biblioteca comunale Sirio Giannini e l'Archivio storico, della cui gestione mi occupo da quasi dodici anni, sono ubicati al pianterreno del cinquecentesco Palazzo Mediceo di Seravezza, dal 2013 patrimo- 
nio UNESCO. Collocata ai piedi delle Alpi Apuane, Seravezza è una città dell'alta Versilia, in provincia di Lucca, che conta circa 14.000 abitanti, nota soprattutto per il marmo estratto dalle proprie cave.

La Versilia è una zona conosciuta e molto apprezzata dal punto di vista turistico e culturale, è quello che il linguaggio del mondo del marketing potrebbe definire un brand. Questo da un lato garantisce la presenza sul territorio di una potenziale e vasta utenza, mentre dall'altro crea una forte concorrenza tra i numerosi eventi che ogni anno sono organizzati dai suoi comuni, una concorrenza dalla quale biblioteche e archivi rischiano di uscire penalizzati.

La biblioteca comunale di Seravezza vanta un patrimonio di circa 33.000 volumi (solo pubblicazioni moderne) e 700 documenti multimediali.

L'archivio storico nasce dallo smembramento dell'archivio del Capitanato di Pietrasanta e consta di circa 3.000 unità, distinte tra sezione preunitaria e sezione postunitaria. Il documento più antico conservato presso l'archivio è uno statuto del comune di Cappella del 1542.

Il territorio in cui operiamo, la sua storia, il relativo contesto, gli strumenti, le risorse umane e economiche a disposizione, l'utenza reale e potenziale sono tutti aspetti che devono essere considerati per una pianificazione strategica di promozione di entrambi gli istituti, che sia concreta ed efficace in tempi relativamente brevi.

Pur essendo una bibliotecaria consapevole delle evidenti differenze tra biblioteca e archivio, la necessità di studiare, programmare e organizzare 'in contemporanea' eventi volti alla valorizzazione culturale di queste due realtà mi ha portato spesso a considerarle come due facce della stessa medaglia, una sorta di Giano Bifronte. Sono infatti entrambi luoghi della memoria e della cultura destinati alla conservazione e alla fruizione, quest'ultima soprattutto per la parte legata alla consultazione, seppur ciascuno con peculiarità proprie che presuppongono professionalità specifiche e che si rivolgono a tipologie di utenza quasi sempre diverse.

\subsection{La biblioteca social: i progetti realizzati presso la comunale di Seravezza}

Gli eventi organizzati dalla biblioteca comunale Sirio Giannini sono proposti e pubblicizzati, non solo tramite il sito istituzionale del comune di Seravezza, ${ }^{1}$ ma anche e soprattutto attraverso l'utilizzo dei

1 Per sito istituzionale del Comune di Seravezza vedi: https://www.comune.seravezza.lucca.it/c@46028/zf/index.php/servizi-aggiuntivi/index/index/idtesto/15 
social: Facebook ${ }^{2}$ (public group utenti della biblioteca) e Instagram. ${ }^{3}$ All'inizio questi canali di nuova generazione erano utilizzati alla stregua di una bacheca degli avvisi, ma nel tempo sono diventati un valido strumento di promozione della lettura e della biblioteca. Alcune manifestazioni sono state organizzate appositamente per la dimensione social, una di queste è senza dubbio il Mannequin challenge realizzato nel 2017 in occasione della Giornata mondiale del libro e del diritto d'autore. Un Mannequin challenge è un fenomeno di internet, una competizione che ha spopolato in rete che nasce nel 2016 negli Stati Uniti in ambito scolastico e consiste nella ripresa breve di persone immobili come una sorta di fermo immagine. Per individuare la modalità di realizzazione più adatta al nostro contesto ne sono state esaminate diverse tipologie, soprattutto eseguite in biblioteche statunitensi e australiane. Il Mannequin challenge proposto dalla biblioteca di Seravezza si ispira alla rielaborazione dei dieci diritti del lettore di Daniel Pennac cui ci siamo permessi di aggiungerne per l'occasione un undicesimo: il diritto di leggere in qualunque momento e in qualunque posa. L'evento è stato organizzato a costo zero: i protagonisti, tutti volontari, sono stati soprattutto utenti della biblioteca, che con entusiasmo hanno accolto la proposta di partecipare alla manifestazione. Il video è stato caricato su YouTube ${ }^{4}$ e poi promosso attraverso i social tramite i quali ha ottenuto numerose visualizzazioni e condivisioni. Sono stati raggiunti diversi obiettivi: la promozione della lettura e della biblioteca come ambiente e come sede, ma soprattutto il progetto ha messo in risalto il ruolo attivo degli utenti come protagonisti dell'evento, attorno ai quali ruota l'intero universo della biblioteca di pubblica lettura.

Dal 2014 la biblioteca Sirio Giannini ospita gli alunni delle scuole superiori convenzionate con il Comune per i progetti di tirocinio e, attualmente, quelli di Alternanza Scuola Lavoro. Durante le ore in cui gli studenti e le studentesse si avvicinano al mondo della biblioteca vengono introdotti dal personale alle relative mansioni: dal prestito alla gestione inventariale e alla consultazione. Sono stati poi invitati a dare il loro contributo per migliorare le pagine social utilizzate e per creare eventi tramite web. Fra questi è stato organizzato il concorso Biblioselfie: fatti un selfie in biblioteca! Gli utenti sono stati invitati a scattare un selfie con un libro (preferibilmente in mano) in una delle sezioni della biblioteca (o anche negli spazi esterni) e a postare le foto sui social [fig. 1]. La foto che ottiene il maggior numero

2 Per public group della Biblioteca di Seravezza vedi: https://www.facebook.com/ groups/104124412955454/.

3 Per la pagina Instagram della Biblioteca di Seravezza vedi: @biblioseravezza.

4 Per il mannequin challenge della Biblioteca di Seravezza vedi: https://www.youtube. com/watch?v=ripXV5rtSi8. 


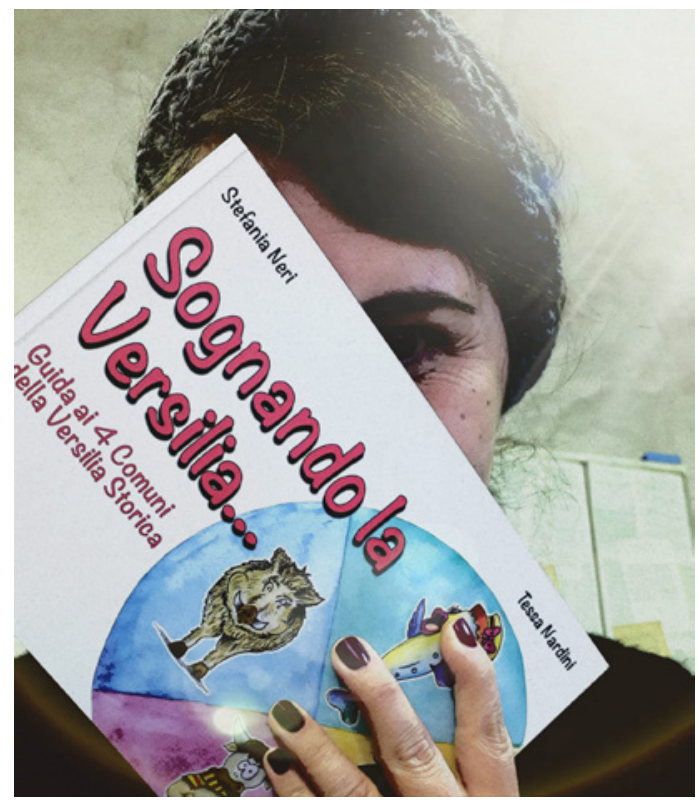

Figura 1 Biblioselfie:

fatti un selfie in biblioteca! (c) Stefania Neri

di like (e l'utente che l'ha realizzata) vince: il premio consiste naturalmente in libri. I ragazzi hanno redatto un vero e proprio bando di partecipazione e per celebrare degnamente i vincitori di ogni categoria (miglior selfie femminile, maschile, di gruppo, etc.) è stato organizzato un evento ad hoc con la consegna di premi e attestati. In questo caso la promozione avviene a livello sia culturale che turistico: nei post infatti, oltre ai libri e alle sale della biblioteca si possono ammirare anche scorci del Palazzo Mediceo e del parco antistante.

Sono stati realizzati altri due progetti con la collaborazione degli studenti dell'Alternanza Scuola Lavoro: In biblioteca con l'utente e Il Bibliocalendario. Il primo è basato su una breve intervista (3 minuti circa) a un utente curata dagli stessi ragazzi che si sono improvvisati giornalisti e registi. Le domande riguardano la lettura e la biblioteca: autore e libro preferiti, numero di libri letti durante l'anno, evento/i della biblioteca più amati, suggerimenti per migliorare il servizio. I video delle interviste, sono stati caricati sui relativi social per essere condivisi. Anche in questo caso tutti i partecipanti, intervistatori, intervistati e registi, hanno ricevuto libri in omaggio.

Il Bibliocalendario è stato realizzato tra il 2016 e il 2017 prima in forma cartacea e poi digitale: ciascuna delle dodici immagini è diventata lo sfondo della pagina Facebook della biblioteca per i mesi dell'anno. I soggetti delle fotografie sono stati proprio i giovani e le 
Michela Corsini

I luoghi della memoria 2.0. Strategie di promozione social e digital della biblioteca e dell'archivio
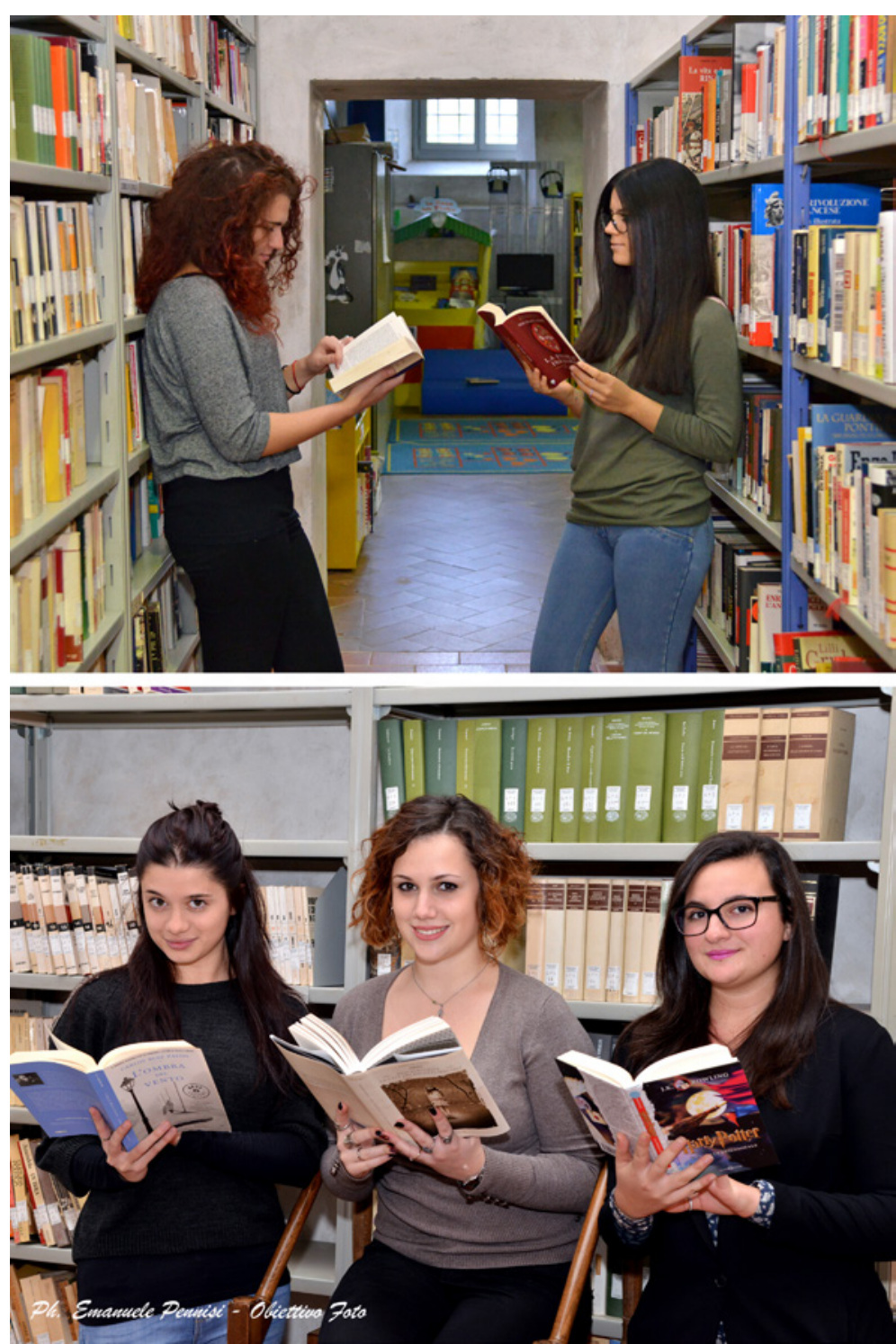

Figura 2 Bibliocalendario. (c) Emanuele Pennis 


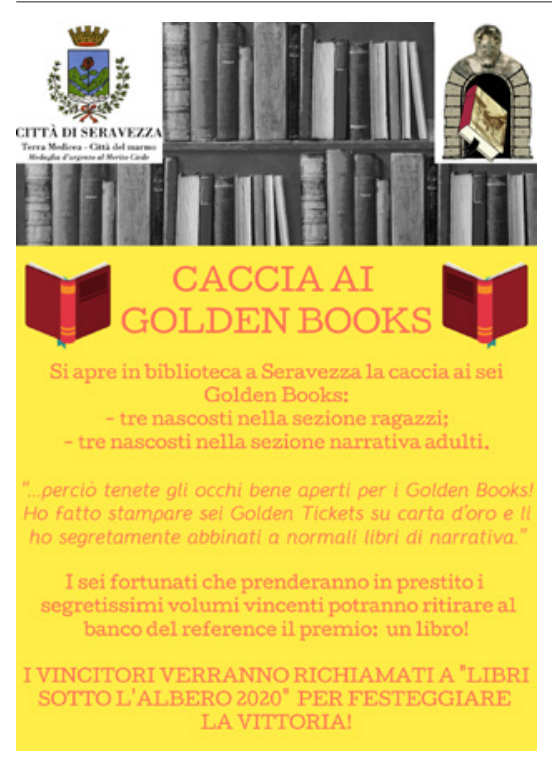

Figura 3 Caccia ai Golden Books. (C) Elisa Garfagnini

giovani dell'Alternanza Scuola Lavoro, immortalati tra gli scaffali o dietro al bancone del reference. Con queste immagini è stato realizzato anche un video che ancora oggi è utilizzato dal liceo di provenienza degli studenti per la promozione dell'Alternanza Scuola Lavoro in biblioteca [fig. 2].

Per quanto concerne la parte relativa ai progetti proposti sul web della biblioteca comunale concludiamo con la Caccia ai Golden Books, un nuovo esperimento che da poco è stato realizzato tramite social. L'idea prende le mosse dal romanzo La fabbrica di cioccolato di Roald Dahl: sei preziosi golden tickets sono racchiusi virtualmente in altrettanti golden books della biblioteca (abbinati al relativo numero d'inventario), tre nella sezione della narrativa adulti e tre nella sezione dei bambini e ragazzi. Gli utenti dovranno indovinare di quali opere si tratta. Ogni settimana su Facebook e Instagram viene dato un indizio (solitamente relativo a uno dei protagonisti del libro) per poterli individuare. Il lettore che pensa di aver indovinato deve recarsi in biblioteca e chiedere quel libro in prestito, se è effettivamente uno del golden books riceve immediatamente un volume in omaggio [fig. 3].

Come evidente, insieme ai laboratori didattici di promozione della lettura, quali Nati per Leggere e Nati per la Musica, che mensilmente vengono proposti alla nostra utenza, abbiamo deciso di percorrere anche la strada dell'innovazione per una - speriamo - migliore promozione della biblioteca. 


\section{L'archivio storico comunale tra didattica e digitalizzazione}

La promozione della documentazione afferente l'archivio storico comunale avviene tramite canali diversificati: la stampa di pubblicazioni specialistiche, tra le quali molto importante è l'inventario della documentazione preunitaria; l'esposizione di unità archivistiche restaurate e/o di particolari tipologie documentarie per le quali sono stati redatti i relativi inventari; visite guidate per gli studenti degli istituti scolastici presenti sul territorio e per gruppi di turisti e persone interessate a vario titolo, che si concentrano in particolari occasioni e ricorrenze quali la Settimana della Cultura e le Giornate Europee del Patrimonio. Negli ultimi anni queste modalità di promozione dell'archivio, e gli eventi che ne scaturiscono, sono state arricchite e integrate da altre proposte più innovative, soprattutto dedicate alla didattica e alla digitalizzazione.

All'interno dei progetti realizzati tramite la Rete Documentaria Lucchese, alla quale aderiscono 57 istituti tra biblioteche e archivi, sono stati organizzati nell'area versiliese una serie di laboratori didattici gratuiti rivolti ai bambini tra i 5 e i 9 anni e ai ragazzi tra i 10 e i 14 anni con l'obiettivo di spiegare la funzione e l'importanza dell'archivio, specificatamente dell'archivio storico comunale. A questo proposito sono state coinvolte le scuole del territorio, primarie di primo e secondo grado, tramite l'Istituto Comprensivo di riferimento. La realizzazione dei laboratori è stata affidata a operatrici specializzate in didattica culturale e visite guidate teatralizzate. Per i più piccoli, ad esempio, è stato appositamente inventato il personaggio di Filomena la Pergamena, impersonato da una delle curatrici del laboratorio, che spiega ai bambini in maniera semplice che cosa è un archivio e che tipo di documentazione conserva e li invita a creare, tramite i loro disegni, uno stradario simile a quello del 1784 conservato in $\operatorname{archivio}^{5} \mathrm{e}$ del quale è stato realizzato un facsimile. Per i più grandi invece il laboratorio si svolge in maniera più approfondita relativamente al concetto di bene culturale della documentazione archivistica, alla storia del territorio versiliese e ai personaggi della famiglia Medici ad essa legati: il Granduca Cosimo I, la Regina Caterina de' Medici e la Granduchessa Maria Cristina di Lorena. Va da sé che le classi che aderiscono ai laboratori didattici visitano l'archivio storico comunale entro la fine dell'anno scolastico [fig. 4].

Per quanto concerne la digitalizzazione l'Amministrazione comunale dal 2016 ha intrapreso un percorso volto alla digitalizzazione di tutta la documentazione archivistica della Seconda guerra mon-

5 Comunità di Seravezza Cancelleria di Pietrasanta Campione di Strade 1784. Cf. Allegranti 2015, 115. 


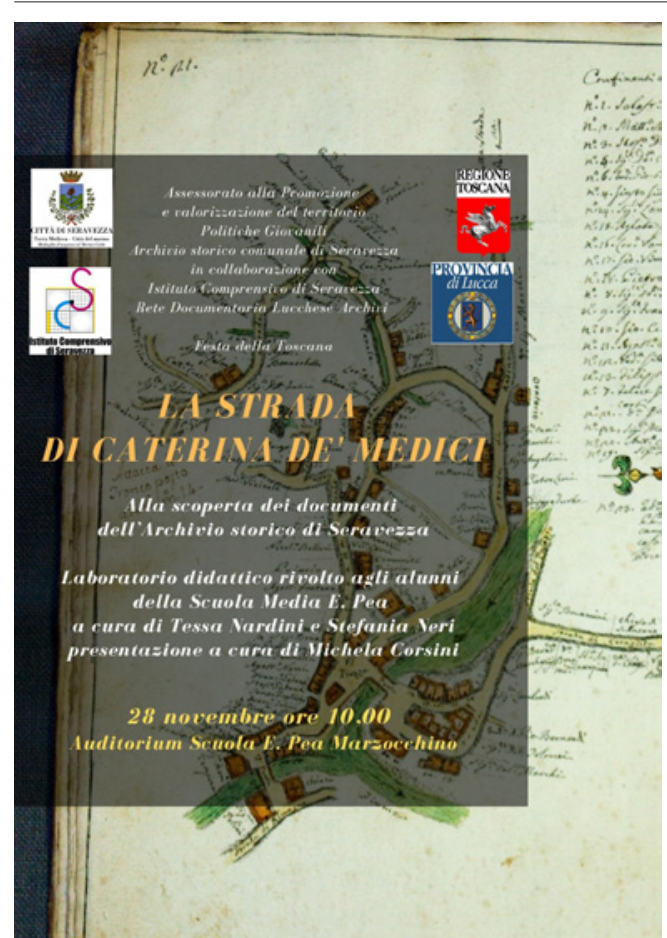

Figura 4 Lastrada di Caterina de'Medici. Laboratorio didattico. Locandina promozionale. (c) Stefania Neri

diale (soprattutto i manifesti) e della Fototeca storica della Versilia. Quest'ultima si compone di una collezione di foto e cartoline (circa un migliaio) risalente a un periodo che va dai primi anni del Novecento fino agli anni Sessanta e Settanta, di cui 700 sono state digitalizzate a una risoluzione di $300 \mathrm{dpi}$. Le immagini sono state dotate di filigrana elettronica, e poi catalogate tramite software Sebina Next in modo che possano essere consultabili tramite l'OPAC della Rete Documentaria Lucchese e anche su dispositivi mobili grazie all'app di BiblioLucc@. ${ }^{6}$ Le digitalizzazioni in alta risoluzione e senza filigrana sono a disposizione per eventuali pubblicazioni previa richiesta all'ufficio di competenza. Una parte delle immagini della fototeca, soprattutto quelle con soggetti relativi ai mestieri legati al mondo delle cave, dell'agricoltura e allevamento, è stata utilizzata per la creazione di un video caricato su una postazione multimediale del Museo del lavoro e delle tradizioni popolari della Versilia storica a disposizione dei visitatori e degli studenti delle scuole. 
Infine un breve accenno merita anche la sezione del sito del Comune dedicato all'archivio storico dove è stata creata una digital library $^{7}$ attraverso la quale si possono consultare da remoto alcuni tra i documenti più significativi del patrimonio documentario comunale tra i quali l'estimo ${ }^{8}$ del 1576 composto da oltre 900 carte.

\section{Bibliografia}

Allegranti, B. (2015). L'archivio del Comune di Seravezza. Sezione Preunitaria e Opere di chiese, conventi e compagnie religiose. Introduzione e inventario. Seravezza: Comune di Seravezza.

7 Per accedere alla digital library del Comune di Seravezza vedi: https://www.comune.seravezza.lucca.it/c046028/zf/index.php/servizi-aggiuntivi/index/index/idtesto/48.

8 Seravezza Estimo 1576. Cf. Allegranti 2015, 80. 


\title{
Oltre le mostre
}

a cura di Mauro Brunello, Valentina De Martino e Maria Speranza Storace

\section{I codici si mostrano Esperienze nella Biblioteca Malatestiana}

\section{Paola Errani}

Biblioteca Malatestiana, Cesena, Italia

\begin{abstract}
The Biblioteca Malatestiana was founded in 1452 by Malatesta Novello, lord of Cesena. Since then not only its architecture, but also its furniture and manuscripts have been perfectly preserved. Therefore visitors can still admire its manuscripts in their original setting. In order to meet the educational needs of pupils and students for many years we offered special events, including guided tours and laboratories, specifically designed for primary schools, secondary schools and universities. Since 2014 we joined the Associations of Friends of the Malatestiana Library in organizing lectures concerning individual manuscripts, which during the lectures were exposed to the audience. Since 2016 visitors can get an experience of the ordinary maintenance and conservation treatment of manuscripts: once a month from March to June they have the opportunity to look at book and paper conservators at work. Besides, we experimented special guided tours for blind persons, who were allowed to touch the various parts of manuscripts (i.e. the chain, the cover, the two sides of the parchment leaves).
\end{abstract}

Keywords Malatestiana Library. Manuscripts. Guided tours. Laboratories. Lectures. Conservation treatments.

Sommario 1 La Biblioteca Malatestiana. - 2 Visitare la Malatestiana. - 3 Consultare i codici. - 4 La Malatestiana e la scuola. - 5 I codici si mostrano. - 6 II restauro 'in diretta' dei manoscritti. -7 Prospettive. 


\section{La Biblioteca Malatestiana}

Tra le biblioteche del Quattrocento la Malatestiana si distingue per la eccezionale conservazione dell'edificio, degli arredi e del patrimonio librario. Per questa sua straordinaria integrità e per l'interesse del suo contenuto l'UNESCO nel 2005 l'ha inserita, prima fra le biblioteche italiane, nel Registro della Memoria del Mondo. ${ }^{1}$

Fondata da Malatesta Novello, signore di Cesena dal 1433 al 1465, all'interno del convento di San Francesco, fu affidata dal principe alla cura e alla tutela della municipalità, anche se il custode bibliotecario era scelto tra i frati del convento: di questa doppia custodia sono simbolo le due antiche chiavi con cui tuttora si apre la porta. Dal punto di vista architettonico la sala presenta una pianta basilicale, suddivisa in tre navate da due file di dieci colonne ciascuna, ed è illuminata da ventidue finestrine su ogni lato e da un rosone sulla parete di fondo, che distribuiscono uniformemente la luce. L'architetto fu il fanese Matteo Nuti. Terminati i lavori nel 1452, la biblioteca venne 'inaugurata' ufficialmente «a dì 15 d'agosto 1454», come si legge sulla splendida porta lignea d'ingresso, che reca gli stemmi malatestiani e il nome dell'intagliatore, Cristoforo di San Giovanni in Persiceto.

Altrettanto impegno Malatesta Novello profuse nell'assicurare alla sua biblioteca una cospicua dotazione libraria, commissionando oltre centoventi codici e acquistando manoscritti greci ed ebraici. A questi si aggiunsero i libri appartenenti al convento di San Francesco e quelli donati dal medico del Malatesta, Giovanni di Marco, per un totale di 343 codici. I volumi comprendono testi di teologia e filosofia, di medicina e di scienze, opere degli autori classici latini e greci e dei Padri della Chiesa.

Il vigile controllo esercitato dal Comune nei secoli ha determinato la straordinaria conservazione della biblioteca in tutti i suoi elementi, permettendole di superare indenne la fine della dominazione dei Malatesti, la soppressione in età napoleonica del convento francescano che la ospitava, e tanti altri momenti difficili della sua storia.

Dall'inizio dell'Ottocento il luogo in cui sorgeva l'antica biblioteca è stato oggetto di radicali trasformazioni edilizie, che non hanno però manomesso l'edificio quattrocentesco. I locali già del convento, opportunamente ristrutturati, sono divenuti sede in parte della biblioteca comunale, inaugurata nel 1807 con i fondi librari appartenuti alle congregazioni religiose soppresse nel 1797, in parte del Liceo

1 Della vasta bibliografia sulla Malatestiana si ricordano Campana 1932, 1953; Domeniconi 1960; Ortalli 1982; inoltre gli atti dei convegni tenuti a Cesena nel 1989 e nel 2003: Lollini, Lucchi 1995; Righetti, Savoia 2006. Sui manoscritti anche: Domeniconi 1993 Casamassima, Guasti 1992; Manfron 1998; Errani, Lollini 2002; Errani, Palma 2006. 
classico, fondato nel $1860 .^{2}$ Dal 2006, con il trasferimento del Liceo in un edificio moderno, la Biblioteca ha potuto ampliare i suoi spazi. Attualmente la Comunale possiede un notevole patrimonio librario, che oltre ai libri e ai periodici moderni, comprende carteggi, archivi, stampe e incisioni, fotografie e un importante fondo antico, composto di manoscritti moderni, 300 incunaboli, circa 4.000 cinquecentine e migliaia di edizioni dei secoli XVII-XVIII. ${ }^{3}$

La gestione della biblioteca ha posto tra i suoi obiettivi la tutela e la conservazione del patrimonio librario antico, al fine di trasmetterlo nelle migliori condizioni alle future generazioni, lo studio e la catalogazione, per una sua più approfondita conoscenza, e la sua valorizzazione, nel senso di una sempre maggiore visibilità e opportunità di approfondimento non solo per gli studiosi, i ricercatori, i cosiddetti 'addetti ai lavori', ma per un pubblico più ampio possibile.

In questo intervento si cercherà di descrivere brevemente i percorsi di valorizzazione che si sono messi in atto per quanto riguarda la biblioteca quattrocentesca.

Fin dalla sua fondazione la Malatestiana antica ha avuto la caratteristica di essere sia un luogo di studio sia un monumento da visitare. Le testimonianze sulla sua frequentazione da parte degli studiosi e dei visitatori, purtroppo scarse per quanto riguarda i suoi primi secoli di vita, precise e puntuali invece dagli anni Settanta dell'Ottocento, mostrano come la gestione dell'istituto, consapevole di queste due connotazioni, si sia sempre impegnata nell'organizzazione sia del percorso di visita, sia della consultazione dei codici che vi sono conservati. ${ }^{4}$ Per dare un'idea del numero dei visitatori si riportano i dati relativi agli anni 1871-1925 e 2000-19:

\begin{tabular}{lccccccccccc}
\hline ANNO & $\mathbf{1 8 7 1}$ & $\mathbf{1 9 0 4}$ & $\mathbf{1 9 0 6}$ & $\mathbf{1 9 1 5}$ & $\mathbf{1 9 1 6}$ & $\mathbf{1 9 1 7}$ & $\mathbf{1 9 1 8}$ & $\mathbf{1 9 2 0}$ & $\mathbf{1 9 2 1}$ & $\mathbf{1 9 2 2}$ & $\mathbf{1 9 2 3}$ \\
\hline Nr. visitatori & 118 & 433 & 630 & 181 & 156 & 131 & 23 & 227 & 444 & 292 & 333 \\
\hline ANNO & $\mathbf{1 9 2 4}$ & $\mathbf{1 9 2 5}$ & $\mathbf{2 0 0 0}$ & $\mathbf{2 0 0 1}$ & $\mathbf{2 0 0 2}$ & $\mathbf{2 0 0 3}$ & $\mathbf{2 0 0 4}$ & $\mathbf{2 0 0 5}$ & $\mathbf{2 0 0 6}$ & $\mathbf{2 0 0 7}$ & $\mathbf{2 0 0 8}$ \\
\hline Nr. visitatori & 283 & 686 & 9.762 & 12.215 & 11.476 & 19.836 & 10.402 & 13.736 & 20.350 & 18.224 & 15.018 \\
\hline & & & & & & & & & & & \\
\hline ANNO & $\mathbf{2 0 0 9}$ & $\mathbf{2 0 1 0}$ & $\mathbf{2 0 1 1}$ & $\mathbf{2 0 1 2}$ & $\mathbf{2 0 1 3}$ & $\mathbf{2 0 1 4}$ & $\mathbf{2 0 1 5}$ & $\mathbf{2 0 1 6}$ & $\mathbf{2 0 1 7}$ & $\mathbf{2 0 1 8}$ & $\mathbf{2 0 1 9}$ \\
\hline Nr. visitatori & 18.629 & 16.525 & 18.095 & 14.924 & 14.361 & 16.810 & 18.217 & 22.592 & 19.613 & 21.926 & 11.261 \\
\hline
\end{tabular}

2 Sulla storia della Biblioteca comunale cf. Baldacchini 1992; Errani 2006, 2011. Sul Liceo cf. Mengozzi 2011.

3 Sui fondi della Biblioteca Comunale cf. Savoia et al. 2007.

4 Sulla frequentazione della Malatestiana cf. Errani, Palma 2018. 
La Biblioteca si è valsa, a partire da almeno due decenni, delle molteplici opportunità dischiuse dal digitale per comunicare con efficacia le caratteristiche e il valore di quello che si usava chiamare «materiale raro, antico e di pregio».

\section{Visitare la Malatestiana}

Le visite guidate alla parte storica hanno da sempre costituito il momento privilegiato di incontro con la Malatestiana e con un periodo importante della storia cittadina. Visitare la Malatestiana significa avere la possibilità di osservare una biblioteca umanistica straordinariamente ben conservata, con i codici ancora collocati nell'ambiente in cui furono disposti fin dall'origine. Le visite, in cui si fornisce una spiegazione della storia della Malatestiana e del suo patrimonio bibliografico, fino a poco tempo fa condotte da un operatore della biblioteca, ora sono affidate a una cooperativa culturale, in grado di svolgerle non solo in lingua italiana ma anche in inglese. Sono inoltre disponibili gratuitamente audioguide in inglese e in tedesco. Nel 2004 è stata realizzata lungo il corridoio di accesso alla sala quattrocentesca una mostra introduttiva, che attraverso brevi testi e immagini descrive il personaggio fondatore, Malatesta Novello, la famiglia dei Malatesti, la città al tempo di Malatesta Novello, la fondazione della Biblioteca, i fondi manoscritti in essa conservati.

All'inizio del percorso di visita è stato collocato un touch screen, che permette di sfogliare alcuni codici e vedere una galleria di immagini dei fondi storici conservati in biblioteca. Per un corale appartenente alla serie di quindici libri liturgici del XV secolo, che sono esposti nella sala adiacente alla biblioteca antica, oltre alla visione delle immagini è possibile ascoltare il relativo canto gregoriano. ${ }^{5}$

Nell'atrio della Malatestiana sono esposti due codici a rotazione, per non più di due mesi, scelti di volta in volta per far conoscere le caratteristiche della collezione libraria, in base quindi alla provenienza (manoscritti commissionati da Malatesta Novello, o appartenuti al medico del Novello Giovanni di Marco, o in dotazione alla biblioteca del convento), all'opera contenuta (Bibbie, classici latini, testi di medicina...), alla lingua del testo (codici greci o ebraici).

5 Dei corali presenti in Biblioteca otto furono commissionati negli anni Cinquanta del Quattrocento dal cardinale Bessarione, che intendeva inviarli a Costantinopoli al convento di Sant'Antonio de Cyprissis. Caduta la città in mano ai Turchi, Bessarione li donò al convento cesenate dell'Osservanza. I volumi erano una quindicina, ma sette andarono dispersi al tempo delle soppressioni napoleoniche. L'altra serie, costituita da sette corali, fu scritta dal copista Enrico di Amsterdam nel penultimo decennio del XV secolo su incarico del vescovo di Cesena Giovanni Venturelli. Di proprietà della Cattedrale, è in deposito dal 1919 presso la Biblioteca. Cf. Lucchi 1989. 
Il percorso svolto prima di entrare nella Malatestiana intende quindi far arrivare i visitatori più 'preparati' e consapevoli di quello che andranno a osservare, fermo restando che niente può sostituire la visione diretta di quell'ambiente di incredibile fascino e suggestione.

È stato sperimentato, in collaborazione con l'Associazione Unione Italiana dei Ciechi e Ipovedenti, sezione di Forlì, un percorso di visita alla Malatestiana per i non vedenti, che hanno potuto toccare alcuni elementi architettonici e di arredo e le varie parti di un codice (la catena, la coperta, i due lati di un foglio di pergamena). Nei contesti museali che stanno sperimentando questa innovazione, gli strumenti utilizzati consistono essenzialmente di libri scritti in Braille o a caratteri ingranditi, di mappe tattili, percorsi audioguidati, strisce di segnalazione a terra, oltre alle spiegazioni fornite dal personale addetto; nel caso della visita alla Biblioteca e ai suoi codici, non solo occorrerebbe introdurre questi strumenti, ma organizzare i modi più idonei per l'esplorazione tattile di manoscritti e di elementi architettonici.

Infine è recentissima la messa in rete di una mostra sull'antica biblioteca dal titolo Biblioteca Malatestiana. Una storia per immagini. Essa si propone di costituire un percorso virtuale - principalmente iconografico - all'interno di un'area della città di Cesena, particolarmente ricca di storia e di avvenimenti quale appunto la biblioteca e le sue adiacenze. La mostra è anche l'occasione per far conoscere fotografie e cartoline poco note, in alcuni casi addirittura inedite, come le tante cartoline d'epoca del 'Giardino Bufalini', gli scatti realizzati nel sottotetto della Malatestiana e le suggestive immagini dei restauri realizzati negli anni Venti del Novecento. Il percorso digitale è cosparso di link che guidano il lettore verso alcuni approfondimenti, e non mancano articoli di quotidiani storici, per accompagnare le immagini d'epoca con racconti e commenti coevi. ${ }^{6}$

\section{Consultare i codici}

Insieme all'organizzazione delle visite guidate si è posto attenzione, come si è detto, anche alle modalità di consultazione dei manoscritti e libri antichi. La consultazione ha luogo in un'apposita sala, fornita di telecamere di sorveglianza e alla presenza di un bibliotecario. Si è sempre

6 La mostra è stata ideata e realizzata dalla bibliotecaria della Malatestiana Carla Rosetti, autrice di altre due esposizioni virtuali: Saluti da..., che illustra la raccolta di cartoline di primo Novecento, conservate nel fondo Comandini della Biblioteca (http:// mostre.malatestiana.it/cartoline) e Archivio fotografico Giuseppe Ambrosini, che attraverso le foto del giornalista sportivo presenta un veloce excursus sulla storia del ciclismo italiano (http://mostre.malatestiana.it/ambrosini). 
cercato di coniugare il più rigoroso rispetto delle norme di tutela e di conservazione dei codici con la disponibilità a venire incontro alle esigenze degli studiosi e ricercatori: non si è limitata la visione dell'originale per favorire quella della riproduzione, né si sono posti dei limiti al numero dei codici richiesti per lo studio. Alla consultazione dal vivo si è voluto affiancare quella 'virtuale', con funzioni e obiettivi ovviamente diversi. Nel corso delle celebrazioni del $550^{\circ}$ di fondazione della Malatestiana (1452-54 - 2002-04) nel 2002 è stato promosso il Catalogo aperto dei manoscritti malatestiani, tramite il quale è possibile effettuare visite 'virtuali' alla biblioteca e al suo patrimonio. ${ }^{7}$ Il catalogo offre testi fondamentali per la storia della Malatestiana, descrizioni dei manoscritti, una bibliografia continuamente arricchita di nuove notizie e infine la riproduzione integrale dei codici. ${ }^{8}$ E appena il caso di osservare come la messa in rete delle immagini costituisca un vantaggio notevole per gli studiosi (paleografi, codicologi, storici dell'arte...), evitando loro spostamenti materiali e permettendo di consultare quanti manoscritti vogliono, ingrandendo le immagini a piacere e mettendole simultaneamente a confronto. È altresì importare ricordare che l'utenza non è costituita soltanto dagli studiosi, ma anche da appassionati del settore, che possono usufruire della possibilità di 'sfogliare' virtualmente i codici e conoscere il patrimonio custodito nella nostra biblioteca. Tra l'altro la disponibilità dei codici online consente di fruire dei preziosi contenuti della Biblioteca anche in un periodo di emergenza come questo, in cui da un lato musei e luoghi di cultura sono chiusi al pubblico al fine di limitare la diffusione del contagio di Covid-19, e dall'altro un più vasto pubblico, proprio in quanto costretto in casa, è motivato e stimolato a ricercare ed apprezzare simili occasioni culturali. In questo modo, con l'iniziativa denominata «\#Io Resto a Casa... i manoscritti della Malatestiana sfogliabili online» la Biblioteca ha aderito alla campagna culturale della Regione Emilia-Romagna «\#IoRestoaCasa... e leggo».

\section{La Malatestiana e la scuola}

Da anni si è posto una cura particolare per avvicinare la biblioteca antica e il suo patrimonio al mondo della scuola. Con l'ausilio di un'associazione culturale si sono creati dei percorsi didattici adatti alle varie fasce d'età a partire dalla scuola materna, di cui si presenta qui la sintesi. La attività didattiche comprendono sia la visita alla Malatestiana sia un laboratorio.

7 Accessibile all'indirizzo http://www.comune.cesena.fc.it/malatestiana/catalogo-dei-manoscritti.

8 Sul Catalogo aperto cf. Cartelli et al. 2006; Cartelli et al. 2007; Cartelli et al. 2009; Cartelli et al. 2011. 

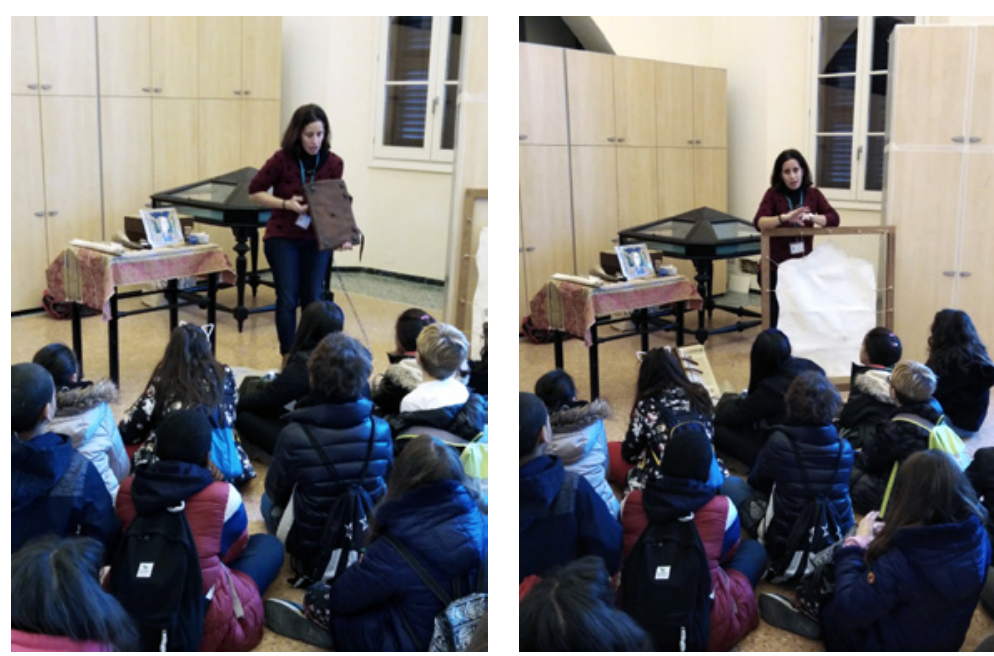

Figura 1 Laboratorio didattico con una classe della scuola primaria, Il ciclo: presentazione del facsimile di un codice. Per concessione dell'Associazione culturale Artemisia

Figura 2 Laboratorio didattico con una classe della scuola primaria, II ciclo: spiegazione della preparazione di un foglio di pergamena. Per concessione dell'Associazione culturale Artemisia

Per la scuola primaria, I ciclo, il laboratorio prevede la realizzazione da parte di ciascun bambino della 'carta d'identità' della Biblioteca in forma di piccolo libro, costituito da un fascicoletto con immagini incollate, che viene poi rilegato con una copertina, con un capolettera da colorare. Per il II ciclo, partendo dal racconto del copista francese Jean d'Épinal, attivo a Cesena alla corte di Malatesta Novello, i partecipanti, dopo la visita all'antica biblioteca, possono osservare materiali didattici appositamente ideati (strumenti di scrittura, pergamene, pennelli, pigmenti e un codice scomponibile) che svelano loro i segreti di bottega, le tecniche e le personalità degli artisti e artigiani che hanno contribuito a creare lo straordinario patrimonio della Malatestiana [figg. 1-2]. La classe si trasforma poi nello scriptorium malatestiano e ciascun alunno realizza un segnalibro, in cui copia una frase di uno scrittore per l'infanzia, utilizzando la scrittura gotica o umanistica, con pennini ed inchiostri [fig. 3].

Per le scuole secondarie di I grado vengono approfondite le tecniche di realizzazione di un codice: preparazione del foglio, scrittura, decorazione miniata e legatura, utilizzando materiali didattici appositamente ideati (strumenti di scrittura, pergamene, pennelli, pigmenti e un codice scomponibile). Al termine della visita gli studenti diventano per una mattina copisti e realizzano un segnalibro, in cui copiano una frase di autori della letteratura classica o contemporanea, utilizzando la grafia gotica o umanistica, con penne d'oca, pennini ed inchiostri. 


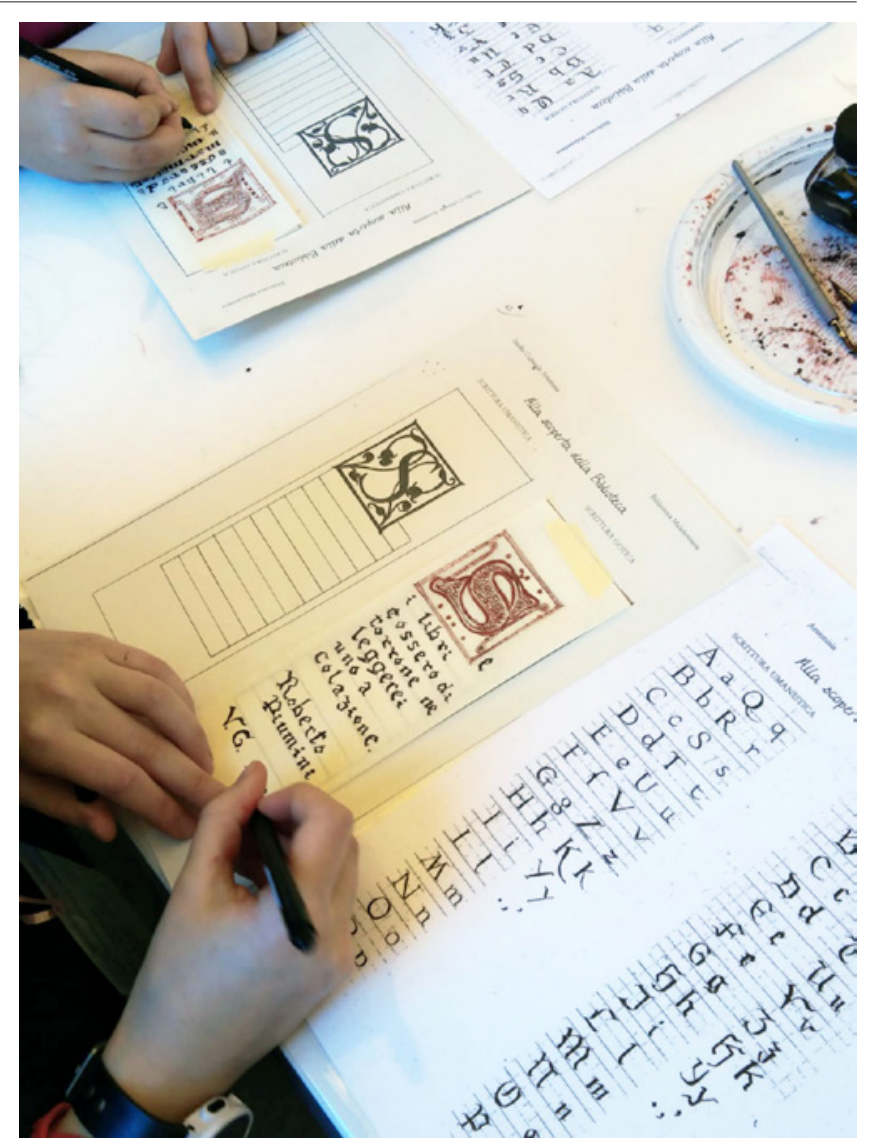

Figura 3 Gli alunni della scuola primaria partecipanti al laboratorio realizzano un segnalibro utilizzando la scrittura gotica. Per concessione dell'Associazione culturale Artemisia

Le scuole secondarie di II grado possono scegliere fra tre tipi di percorso didattico. Il primo intende illustrare le tecniche e le professionalità che ruotano intorno alla creazione degli antichi codici, grazie a materiali didattici appositamente ideati (strumenti di scrittura, pergamene, pennelli, pigmenti e un codice scomponibile) e comprende un laboratorio di calligrafia, in cui ciascuno studente realizza un segnalibro, utilizzando la grafia gotica o umanistica, con penne d'oca, pennini ed inchiostri. Nel secondo percorso, partendo dalla data di inaugurazione della Malatestiana (1454), si racconta che cosa avveniva a Cesena alla corte di Malatesta Novello e cosa contemporaneamente accadeva a Magonza nell'officina di Gutenberg; alla visita guidata alla biblioteca segue una lezione multimediale di approfondimento sulle tecniche e i materiali della stampa ed un laboratorio 
in cui ciascun studente realizza un proprio ex libris, stampato su cartolina, per mezzo di un piccolo torchio. Nel terzo, infine, si approfondisce la storia del passaggio dal manoscritto al libro a stampa e dell'evoluzione delle forme del libro, includendo sia una lezione multimediale sulle tecniche e i materiali della stampa, sia la visione diretta di codici, incunaboli, cinquecentine ed esemplari a stampa dei secoli XVII e XVIII.

La seguente tabella mostra i dati della partecipazione delle scuole alle attività didattiche proposte dalla Biblioteca negli ultimi anni:

\begin{tabular}{lcc}
\hline Anno & Nr. classi & Nr. alunni \\
\hline 2016 & 121 & 3025 \\
\hline 2017 & 142 & 3500 \\
\hline 2018 & 134 & 3350 \\
\hline 2019 & 112 & 2640 \\
\hline
\end{tabular}

Per gli studenti universitari si organizzano insieme ai docenti seminari di approfondimento di paleografia, o di codicologia, o di storia della miniatura, in cui dopo la visita alla Malatestiana, i codici scelti dal docente vengono mostrati e spiegati agli studenti.

\section{$5 \quad$ I codici si mostrano}

La Malatestiana ha organizzato in varie occasioni mostre dei suoi codici, che hanno riscontrato un certo successo e suscitato interesse nei visitatori, pur presentando quei limiti, che il Convegno «Oltre le mostre» si è proposto di focalizzare e superare: si ricordano Scritte dal dito di Dio, esposizione di testi biblici manoscritti e a stampa posseduti dalla Biblioteca (13 aprile-30 giugno 2002) e Malatesta Novello magnifico signore, mostra dei codici malatestiani tra i più rappresentativi dell'intera collezione (dicembre 2002-marzo 2003). Dal 2014 sono iniziate presentazioni dei manoscritti a un pubblico adulto in collaborazione con l'Associazione Amici della Biblioteca Malatestiana. Si tratta di un ciclo periodico di conferenze intitolato «Viaggio fra i tesori della Malatestiana». Nei primi cicli si sono mostrati materiali librari e documentari conservati in biblioteca, a partire dai codici malatestiani, cui hanno fatto seguito la presentazione delle varie collezioni librarie, delle stampe, dei fondi fotografici. In ogni incontro era esposta su un tavolo una scelta di manoscritti o di libri a stampa o di documenti, che il pubblico poteva guardare da vicino, senza 'barriere', e sfogliare insieme al bibliotecario. Nelle conferenze tenute più di recente si sono affrontati temi di letteratura o di storia che potevano fornire l'occasione per mostrare manoscritti: per esempio i codici con le opere di 
Seneca per la conferenza Seneca contro Seneca? Il filosofo e il poeta, i manoscritti greco dell'Odissea e latino con le opere di Virgilio per Ulisse e Enea. Due eroi per due culture, i sette codici ebraici per I fondi ebraici della biblioteca Malatestiana e la presenza degli ebrei a Cesena. ${ }^{9}$ E interessante notare come a proposito dei manoscritti le domande del pubblico si incentrino sulla tecnica di confezione dei codici, sulla modalità di preparazione dei fogli di pergamena, sull'attività del copista e del miniatore, sul tipo di legatura, sulle componenti degli inchiostri e dei colori, sui tempi di realizzazione, come anche sulla storia e sull'uso del codice, rappresentati dalle note di possesso e dai segni lasciati dai lettori, come le caratteristiche maniculae.

\section{Il restauro 'in diretta' dei manoscritti}

Dal 2014 alcuni codici malatestiani sono stati oggetto di interventi di restauro effettuati in sede, cioè all'interno della Biblioteca, in uno spazio appositamente attrezzato, salvo per pochi volumi, per i quali si è reso necessario il trasporto in un laboratorio fuori dalla città. Il restauro dei manoscritti è stato finanziato da privati cittadini e da enti e associazioni locali, che hanno potuto usufruire dell'istituto dell'Art Bonus. ${ }^{10}$ Si è constatato da subito il grande coinvolgimento dei cesenati, che hanno aderito e sostenuto l'iniziativa, ed è nata l'idea di far conoscere loro come si svolge tale attività, organizzando quello che è stato chiamato «il restauro 'in diretta' dei manoscritti». A partire dal 2016 per un sabato al mese nel periodo da marzo a giugno il restauro viene effettuato in una sala della biblioteca a cui il pubblico può accedere, potendo quindi vedere gli interventi sui codici effettuati dalle restauratrici e fare domande sui materiali e sulle tecniche impiegate, oltre ad avere l'occasione di osservare da vicino i manoscritti [figg. 4-5].

9 Qualche titolo delle conferenze, fra le 25 tenute sinora: I codici della Malatestiana; Catullo rivisitato in un codice della Biblioteca Malatestiana; La satira in Lucilio e in Orazio; Malatestiana, uno scrigno di tesori antichi tra ieri e domani; La Biblioteca Piana; Storici e cronisti locali in Biblioteca Malatestiana tra '400 e '800; Il prezioso Decretum Gratiani; I manoscritti dei grandi poeti e scrittori del Novecento custoditi in Malatestiana.

10 Ci si riferisce al Decreto legge 83/2014, convertito con modificazioni in Legge n. 106 del 29/07/2014, che ha introdotto un Bonus a favore di chi contribuisce alla conservazione e allo sviluppo della cultura in Italia. Imprese e privati che effettuano donazioni per iniziative a tutela del patrimonio culturale e per lo sviluppo della cultura possono beneficiare di un credito di imposta al 65\%. Dal 2014 al 2019 sono stati restaurati 62 codici. 

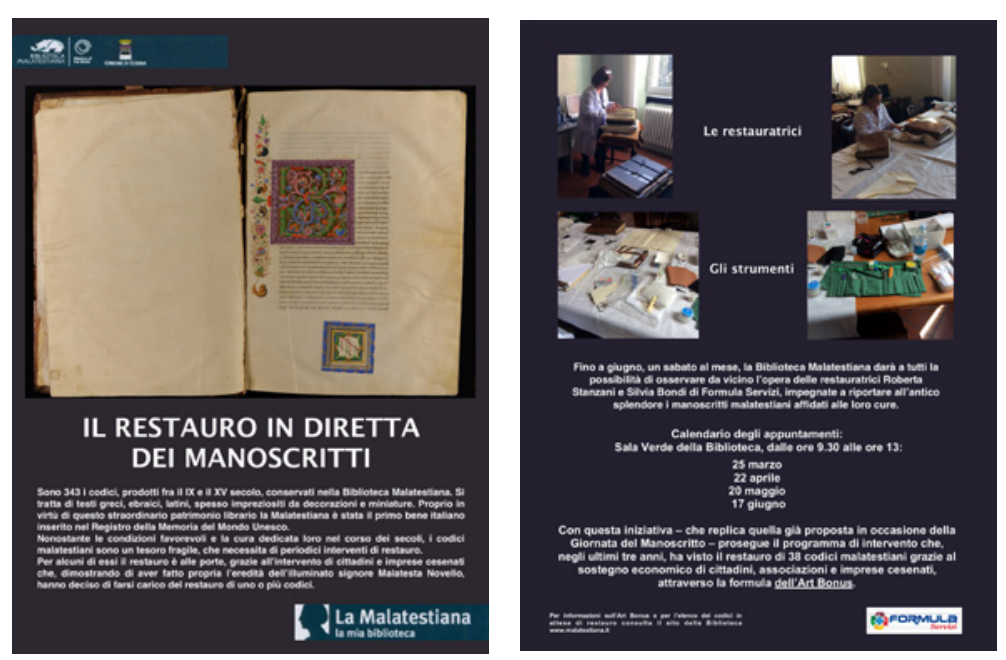

Figure 4-5 Depliant realizzato dalla Biblioteca per pubblicizzare l'iniziativa Restauro in diretta dei codici Malatestiani. @ Paola Errani

\section{$7 \quad$ Prospettive}

Dunque anche in Malatestiana si è già fatto qualcosa per render possibile agli utenti una fruizione a $360^{\circ}$ del patrimonio senza contemporaneamente metterlo a rischio. Molto però resta ancora da fare, che la natura del problema rende difficile anche immaginare al momento. Possiamo però attenerci a un metodo: osservando il continuo mutare della realtà, ascoltando con attenzione i segnali che provengono dagli utenti indicandone le nuove esigenze, seguendo con curiosità e fantasia il progresso delle tecnologie, prenderne spunto per migliorare le tipologie e le caratteristiche dei servizi rendendoli il più possibile in linea con le aspettative. Al fondo, la consapevolezza che «il patrimonio, per noi, non è un mero scrigno di cose: è un ambito di conoscenza, è un motore di innovazione intellettuale» (Balzani 2019): ed è in questa accezione che occorre promuoverlo a vantaggio di tutti. 


\section{Bibliografia}

Baldacchini, L. (1992). «Dalla Libraria domini alla biblioteca pubblica». Baldacchini, L. (a cura di), La Biblioteca Malatestiana di Cesena. Roma: Editalia, 119-66.

Balzani, R. (2019). «Parlare di patrimonio culturale», IBC 27, n. 2.

Campana, A. (1932). «Biblioteche della provincia di Forli». Fava, D. (a cura di), Tesori delle biblioteche d'Italia. Emilia e Romagna. Milano: Hoepli, 83-130.

Campana, A. (1953). «Origine, formazione e vicende della Malatestiana», Accademie e biblioteche d'Italia, 21(1), 3-16.

Cartelli, A. et al. (2006). «ll catalogo aperto dei manoscritti malatestiani». Bollettino AIB, 46(3), 207-20.

Cartelli, A. et al. (2007). «ll catalogo aperto dei manoscritti malatestiani: bilancio e prospettive». Marchiaro, M.; Zamponi, S. (a cura di), Conoscere il manoscritto: esperienze, progetti, problemi. Dieci anni del progetto Codex in Toscana =Atti del Convegno internazionale (Firenze, 29-30 giugno 2006). Firenze: SISMEL; Edizioni del Galluzzo, 153-63.

Cartelli, A. et al. (2009). «ll catalogo aperto dei manoscritti malatestiani». Rehbein, M.; Sahle, P.; Schaßan, T. (Hrsgg), Kodikologie und Palaeographie im digitalen Zeitalter - Codicology and Palaeography in the Digital Age. Norderstedt: BoD, 13-23. Schriften des Instituts für Dokumentologie und Editorik 2.

Cartelli, A. et al. (2011). «ll catalogo aperto dei manoscritti malatestiani: esperienze e prospettive». Dal Prete, I.; Generali, D.; Monti, M. Teresa (a cura di), Le reti in rete. Per l'inventario e l'edizione dell'Archivio Vallisneri. Firenze: Olschki, 123-32. Biblioteca dell'edizione nazionale delle opere di Antonio Vallisneri 5.

Casamassima, E.; Guasti, C. (1992). «La Biblioteca Malatestiana. Le scritture e i copisti». Scrittura e civiltà, 16, 261-82.

Domeniconi, A. (1960). La Biblioteca Malatestiana. Udine: Doretti.

Domeniconi, A. (1993). «Lo scrittorio malatestiano». Romagna arte e storia, 13(37), 23-80.

Errani, P. (2006). Libri, frati e giacobini. Le vicende della Biblioteca Malatestiana nel periodo francese e la nascita della Biblioteca Comunale (1797-1813). Bologna: Compositori. ERBA 61.

Errani, P. (2011). La Biblioteca Comunale dal 1815 all'Unità: note per una storia. Fabbri, P.G. (a cura di), Le vite dei cesenati, vol. 5. Cesena: Stilgraf, 254-94.

Errani, P.; Lollini, F. (2002). "Scritte dal dito di Dio". Testi biblici e liturgici manoscritti e a stampa della Biblioteca Malatestiana = Catalogo della mostra (Cesena, 2002). Forlì: Edit Sapim.

Errani, P.; Palma, M. (a cura di) (2006). I manoscritti datati della provincia di ForliCesena. Firenze: SISMEL; Edizioni del Galluzzo. Manoscritti datati d'Italia 13.

Errani, P.; Palma, M. (2018). Graffiti malatestiani. Storie di donne, uomini, muri e banchi (secoli XV-XXI). Roma: Viella. Scritture e libri del medioevo 17.

Lollini, F.; Lucchi, P. (1995). Libraria Domini. I manoscritti della Biblioteca Malatestiana. Testi e decorazioni. Bologna: Grafis.

Lucchi, P. (a cura di) (1989). Corali miniati del Quattrocento nella Biblioteca Malatestiana. Milano: Fabbri Editori.

Manfron, A. (a cura di) (1998). La biblioteca di un medico del Quattrocento. I codici di Giovanni di Marco da Rimini nella Biblioteca Malatestiana. Torino: Allemandi. 
Paola Errani

I codici si mostrano. Esperienze nella Biblioteca Malatestiana

Mengozzi, M. (2011). Il Liceo "Monti" (1861-2011). Cesena: Fondazione Cassa di Risparmio di Cesena; Stilgraf. Complementi alla storia di Cesena II. Luoghi 1.

Ortalli, G. (1982). «Malatestiana e dintorni. La cultura cesenate tra Malatesta Novello e il Valentino». Vasina, A. (a cura di), Storia di Cesena. Vol. 2, Il Medioevo, tomo 2, Secoli XIV-XV. Rimini: Ghigi, 129-66.

Righetti, L.; Savoia, D. (2006). Il dono di Malatesta Novello = Atti del Convegno (Cesena, 21-23 marzo 2003). Cesena: Il Ponte Vecchio.

Savoia, D. et al. (a cura di) (2007). La casa dei libri: dalla Libraria Domini alla grande Malatestiana. Per i duecento anni della Biblioteca comunale (18072007). Cesena: Comune di Cesena; Istituzione Biblioteca Malatestiana; Lions club Cesena; Fondazione Cassa di Risparmio di Cesena. 



\title{
Valorizzare i fondi d'autore
}

\section{Anna Manfron}

Istituzione Biblioteche del Comune di Bologna e Biblioteca dell'Archiginnasio, Italia

\begin{abstract}
Private libraries and archives constitute an interesting case study for the enhancement of books and documents that are a direct testimony and mirror of the personality that collected, used and produced them. The general public involvement in the narration of the human and intellectual story of an author - starting from good management practices, conservation and cataloging and descriptive treatment, to make the collections openly accessible to the widest possible audience - can be the key to effectively communicate the value of materials that have taken on a special patrimonial vocation. The aim of this paper is also to show how the special collection exhibitions can play a key role in fulfilling obligations to the general public.
\end{abstract}

Keywords Private libraries. Special collections. Value. Impact. Special collection exhibitions.

Sommario 1 Introduzione. - 2 Valorizzare i fondi d'autore attraverso l'accessibilità. 3 Mostre e nuova conoscenza.

\section{Introduzione}

Cosa sono e qual è la funzione principale delle biblioteche? Penso che la risposta più giusta e appropriata è che le biblioteche sono istituti deputati alla promozione della conoscenza, intesa quest'ultima come strumento indispensabile per l'esercizio dei diritti di cittadinanza. Penso anche che la loro missione è soprattutto quella di contribuire a sviluppare e a diffondere, attraverso l'attività culturale rivolta al territorio e alla comunità di riferimento, la capacità di conoscere e analizzare criticamente il nostro passato e il nostro presente.

Il Manifesto IFLA/UNESCO sulle biblioteche pubbliche ne dà questa fondamentale definizione: 
La biblioteca pubblica, via di accesso locale alla conoscenza, costituisce una condizione essenziale per l'apprendimento permanente, l'indipendenza nelle decisioni, lo sviluppo culturale dell'individuo e dei gruppi sociali. $(1995,1)$

Afferma anche che la biblioteca si occupa di «offrire opportunità per lo sviluppo creativo della persona» (2). ${ }^{1}$ Si tratta quindi di luoghi, patrimoni e servizi pubblici attraverso i quali può trovare attuazione quanto previsto all'art. 3 della Costituzione - quindi fra i principi fondamentali - e cioè «il pieno sviluppo della persona umana». In questo momento storico in cui la conoscenza, il sapere sono sottovalutati - talvolta addirittura disprezzati come inutili orpelli - va ancor più sottolineata l'importanza delle biblioteche come presidi per lo sviluppo della cultura, istituti nei quali la Repubblica esercita la tutela del patrimonio storico - e qui il richiamo va all'art. 9 della Costituzione - rappresentato anche dai documenti conservati nelle biblioteche così come negli archivi; documenti che tramandano l'identità culturale della nazione traghettandola verso il futuro. In un discorso tenuto nel 2003 dall'allora Presidente della Repubblica, Carlo Azeglio Ciampi, egli affermò che la tutela non va concepita in senso di passiva protezione, ma in senso attivo, dinamico, e cioè in funzione della cultura dei cittadini che, fruendo dei beni culturali, hanno la possibilità di migliorare se stessi e quindi la società di cui fanno parte (Montanari 2018, 121-2). Il patrimonio culturale è un bene comune, appartiene a tutti. Non trattandosi di beni di lusso riservati a pochi, è quindi vitale spalancare le porte a tutti, facilitarne in ogni modo l'accesso, garantendo i diritti delle persone più che delle cose di interesse storico e artistico a essere semplicemente conservate. Il fine, dunque, non è la mera tutela di oggetti (fra questi i beni librari e documentari), ma favorire - attraverso la fruizione dei beni culturali - la produzione e la redistribuzione di conoscenza e il pieno sviluppo degli individui.

$\mathrm{Al}$ di là del mero accesso all'informazione coincidente con l'evidenza del dato (documento analogico o digitale che sia), oggi, per la maggior parte delle persone, la conoscenza deriva più facilmente dalla comprensione, interpretazione, elaborazione di fatti ed esperienze proprie e altrui, con processi cognitivi innescati e favoriti da forme di narrazione dialettica e da pratiche di partecipazione attiva. Anche per questo, la teoria biblioteconomica si sta rapidamente evolvendo: da una declinazione documentale - che vedeva la biblioteca essenzialmente come sistema che seleziona, conserva e rende acces-

1 La traduzione italiana del Manifesto IFLA/UNESCO sulle biblioteche pubbliche [online] nella revisione del 1994 (la prima versione risale al 1949 ed era stata rivista precedentemente nel 1972) è stata pubblicata a cura dell'Associazione Italiana Biblioteche. 
sibili documenti - si è passati alla biblioteconomia gestionale degli anni Novanta - incentrata cioè sulla gestione dei servizi per progetti e obiettivi, applicando anche tecniche di marketing e pratiche di misurazione e valutazione - fino ad approdare più di recente all'idea di una biblioteconomia che mette al primo posto la persona, riservando un'attenzione particolare alle modalità più efficaci per offrire a tutti facilità di accesso alla conoscenza. In quest'ottica, quindi, le biblioteche - e sicuramente sarebbe bene che tutti gli istituti culturali considerassero questa nuova prospettiva - diventano non solo e semplicemente luoghi dedicati alla conservazione dei documenti della cultura e del sapere, a disposizione di chi vuole e sa come servirsene, ma soprattutto istituti che attivamente contribuiscono alla crescita e al benessere sociale delle persone tramite la diffusione della cultura (Faggiolani 2019).

Il caso dei fondi d'autore e della loro valorizzazione può trovare senz'altro nuove potenzialità in questa visione che valorizza, anche per il pubblico delle biblioteche, forme di conoscenza attiva e partecipativa.

\section{Valorizzare i fondi d'autore attraverso l'accessibilità}

Rispetto alla categoria generica dei fondi personali, la biblioteca e l'archivio di un autore formano un insieme documentario non solo caratterizzato dall'unitarietà rispetto alla provenienza, ma soprattutto determinato dalla figura di colui che l'ha costituito e al quale si devono principi formativi, generi e tematiche, caratteristiche editoriali, specialità, presenza di libri e documenti prodotti e raccolti in quanto frutto di una particolare attività. ${ }^{2}$ Inoltre, il fondo d'autore rappresenta - nella maniera più visibile - l'interfaccia fra l'atto individuale del lavoro e della creazione intellettuale e la rete di relazioni, lo spazio sociale fatto di interessi, amicizie e conoscenze, all'interno del quale lavoro e creazione intellettuale si sono originati e manifestati. Una volta divenuto patrimonio pubblico, ognuno di questi fondi assume un valore del tutto particolare se considerato nel suo complesso, definito da Luigi Crocetti come «archivio culturale» (1999; 2001, 24), cioè quell'insieme indissolubile di carte, libri e oggetti importante per il mondo della ricerca interessato allo studio del contesto, dell'attività e della produzione dell'autore. ${ }^{3}$

Esso può assumere agli occhi del pubblico più vasto altrettanto valore se vengono trovate e messe in campo modalità e tecniche per

2 Per la definizione vedi Desideri, Calabri 2009; Zagra 2007.

3 Soprattutto la critica genetica francese studia tutti i materiali che non hanno un legame diretto con il testo, ma hanno fatto parte del processo creativo (Ferrer 2001). 
interessare e coinvolgere, creando occasioni di conoscenza attorno a questa particolare categoria di patrimonio. Libri, documenti, fotografie, oggetti si trasformano nelle vestigia di personaggi illustri, di stili di vita, di processi di creazione intellettuale e artistica, sono reperti, testimonianze materiali ma anche icone, oggetti rivestiti di un'aura di autenticità, che assumono un potere evocativo, immaginativo o emotivo, diventando portatori di forma e contenuto nella narrazione biografica della vicenda umana e intellettuale di un autore.

Ogni forma di valorizzazione riservata a questa categoria particolare di beni culturali, però, potrà avvenire soltanto al termine di un processo che deve prevedere prima di tutto buone pratiche di gestione, conservazione e trattamento catalografico e descrittivo. Sono quindi indispensabili competenze specialistiche, condizioni favorevoli e investimenti sufficienti per trattare in modo adeguato e in tempi ragionevoli un fondo d'autore, facendo guadagnare alla biblioteca quel grado di autorevolezza e quella reputazione destinati a crescere in maniera progressivamente proporzionale alla visibilità e alla chiarezza - presso amministratori e opinione pubblica - di missione e ambiti d'azione, ma anche quando i professionisti impegnati in queste azioni vedono rafforzata e riconosciuta la responsabilità sui risultati. Il primo obiettivo è sicuramente l'accessibilità e gli strumenti tradizionali, che consentono l'accesso alla documentazione e garantiscono la possibilità di effettuare ricerche rigorose e mirate, sono il catalogo per il materiale bibliografico e l'inventario per i documenti archivistici, salvo individuare approcci di trattamento speciale, come ho proposto già qualche anno fa sottolineando l'importanza delle relazioni da preservare rispetto a questa tipologia di fondo da trattare come un unico complesso documentario (Manfron 2011, 2012a).

Per quanto riguarda la catalogazione, da qualche tempo nelle biblioteche stiamo assistendo a un radicale cambiamento di prospettiva rispetto al catalogo tradizionale, lo strumento più antico, conosciuto e utilizzato (dalla lista sequenziale creata per sé, all'elenco inventariale, al catalogo a volume, poi a schede, fino a quello elettronico) per reperire l'informazione sull'esistenza delle opere di un autore, delle edizioni di una particolare opera o, al limite, di opere su un autore o su un qualsiasi altro argomento. Il cambiamento in corso, che si estende alla descrizione dell'intero universo dei beni culturali, può apparire così complesso da far pensare alla massima (variamente attribuita a Confucio o a Mao): «c'è grande confusione sotto il cielo: la situazione è eccellente». Sicuramente, cioè, la complessità del contesto catalografico in cui si opera è un dato di fatto e le innovazioni - oggi soprattutto in campo tecnologico - sono inevitabili; perciò conviene vederle come un insieme di opportunità, tra le quali serve saper individuare e scegliere quelle più utili per creare valore e porre rimedio all'«assenza di mappe che guidino l'esploratore» 
(Crocetti 1999, 26). ${ }^{4}$ La relazione fra i dati direttamente disponibili nel web è forse il concetto che più di altri sintetizza le trasformazioni in corso nel settore della catalogazione, dove si sta progressivamente superando l'idea del catalogo come elenco di registrazioni a sé stanti per puntare sempre più sulle relazioni fra i dati che rappresentano le parti di un contesto, il quale risulta più facilmente comprensibile e valorizzabile come fonte di conoscenza.

I fondi d'autore sono, a questo proposito, casi straordinari di contesto da preservare e valorizzare anche in fase di trattamento catalografico: ogni manoscritto, libro, documento, opera d'arte, oggetto culturale, mantiene il vincolo naturale, il legame significativo, la relazione con tutto l'insieme che dà un significato particolare alle singole parti. ${ }^{5}$ Goethe a inizio Ottocento, rimpiangendo l'epoca in cui le opere d'arte restavano nella loro collocazione originaria senza essere depredate per formare i grandi musei nazionali, diceva che:

nulla di ciò che vediamo [...] lo vediamo a sé stante ma in rapporto e in unione con qualche altra cosa che gli sta dietro, sotto, sopra. A volte anche un singolo oggetto ci sembra particolarmente bello e pittoresco, ma non è l'oggetto in sé che provoca in noi questa impressione, bensì è il rapporto in cui noi lo vediamo, il rapporto con ciò che gli è vicino, dietro e sopra, che contribuisce a questa impressione. (Carandini 2017, 58-9)

I professionisti, dunque, che si occupano di fondi d'autore, talvolta di case di artisti e scrittori, possono, più di altri, esercitare in maniera consapevole tutte quelle azioni che - come si legge nell'atto costitutivo del 2012 del coordinamento nazionale Musei Archivi Biblioteche (MAB) - servono a superare: ${ }^{6}$

[Le] criticità dei singoli ambiti disciplinari e di promuovere soluzioni organizzative, normative, tecnico-scientifiche per gli istituti culturali e per il patrimonio culturale in genere.

4 Vedi anche la recensione di D'Alessandro (La Repubblica, 10 ottobre 2017) al libro in traduzione italiana di Kevin Kelly, L'inevitabile (Milano: Il Saggiatore, 2017), e Sangalli 2016, 19-20, dove si sottolinea l'importanza della capacità di volgere in positivo gli stimoli del contesto - contesto economico in questo saggio - in cui si opera.

5 Ho già trattato il tema della catalogazione dei fondi d'autore in Manfron 2017.

6 Citazione da MAB. Atto costitutivo, art. 1. MAB è acronimo per Musei Archivi e Biblioteche, coordinamento nazionale permanente promosso nel 2011 dalle tre associazioni professionali: Associazione Italiana Biblioteche (AIB), Associazione Nazionale Archivistica Italiana (ANAI), International Council of Museums - Comitato nazionale italiano (ICOM Italia). La sensibilità rispetto a questi temi ha iniziato a crescere in Italia all'inizio degli anni Ottanta, quando si cominciarono a conservare e valorizzare nella loro unitarietà biblioteche e archivi degli scrittori. Ricordo, a questo proposito il contributo fondamentale di Pisauri 1988. 
La relazione fra le diverse tipologie di materiali che costituiscono i fondi d'autore (carte manoscritte, testi a stampa, fotografie, opere d'arte, oggetti, arredi ecc.) è un dato di fatto; la necessità dell'intreccio delle competenze professionali per trattarli correttamente è ormai riconosciuta e si è sempre più consolidata la sensibilità circa la tutela e la salvaguardia di testimonianze così preziose e particolari, anche quando sono ascrivibili ad autori contemporanei.

Il passo successivo da attuare è ora quello di considerare i nuovi modelli logici che si stanno affermando in campo catalografico per offrire all'utente che naviga in rete la possibilità di ritrovare le parti e il tutto, riconoscendo le relazioni che creano il contesto. Sono già in fase avanzata riflessioni ed elaborazioni relative ai linguaggi di descrizione e di indicizzazione come:

L'integrazione e l'uniformazione degli elementi descrittivi prodotti da istituzioni di natura diversa e le modalità di codifica e architettura dei dati catalografici prodotti. (Bruni et al. 2016)

Otterremo in questo modo quella «potenza informativa moltiplicata» che può derivare dalla confluenza delle «memorie specifiche» (i cataloghi e gli inventari) «in un'unica memoria generale», come aveva già intuito e auspicato Luigi Crocetti nel 1999. Oggi, i dati liberamente accessibili online, interpretabili da una macchina e collegabili ad altri dello stesso tipo (i LOD, cioè Linked Open Data) rappresentano una delle più importanti risorse per l'arricchimento della conoscenza e per la creazione di nuove connessioni tra le rappresentazioni catalografiche e/o digitali dei beni culturali, migliorando al tempo stesso la loro visibilità. I patrimoni di musei, archivi e biblioteche (opere d'arte e oggetti culturali, pubblicazioni, documenti) diventano informazione digitale, che richiede un trattamento catalografico tale da consentire all'utente di passare con facilità da un libro a un oggetto museale, a un documento archivistico, a una registrazione sonora, messi in relazione fra loro ad esempio da un argomento o dall'autore-soggetto produttore-possessore del fondo e così via.

In campo catalografico il cambiamento più importante deriva dal fatto di avere finalmente messo al centro le esigenze informative dell'utente più che i criteri di descrizione del documento, basati su standard internazionali che si considerano ormai comunemente recepiti e adottati. I Principi internazionali di catalogazione del 2009 indicano infatti l'interesse dell'utente fra gli obiettivi principali (punto 2.1), declinando con questa finalità le funzioni del catalogo che deve anche consentire all'utente di «navigare ed esplorare verso altri cataloghi e in contesti non legati alle biblioteche» (punto 6.5) (IFLA 2016, 12). L'obiettivo deve essere quello di rendere navigabili dagli utenti tutti i tipi di dati catalografici, mettendo in relazione dati bibliografici e di altra natura - come quelli relativi a documentazione 
archivistica e a oggetti culturali - anche se frutto di pratiche descrittive tradizionalmente distanti, perché gli utenti sono ormai abituati a percorsi di ricerca trasversali «basati sulla scoperta di relazioni tra entità» (Bianchini 2015, 184). Questo perché è ormai ineludibile la richiesta di aumentare i punti di accesso diretti alla fonte che può contenere l'informazione desiderata, con un approccio che è stato definito Google-like. Dobbiamo quindi aprirci a prospettive catalografiche del tutto nuove che tengano conto delle potenzialità del web semantico per colmare la distanza fra cataloghi e inventari tradizionali - anche quelli che attualmente descrivono in rete le risorse culturali in tanti data-base catalografici distinti e non comunicanti fra loro - e le modalità di reperimento delle informazioni utilizzate dalla gran parte delle persone che, indipendentemente dalla tipologia del documento che veicola l'informazione cercata, utilizzano i motori di ricerca più comuni come unico punto di accesso per soddisfare curiosità e bisogni informativi, in genere senza andare oltre i primi tre risultati offerti. Certamente questa nuova prospettiva non esclude, anzi esalta i risultati della pluri-decennale attività di catalogazione e descrizione nata a fini di tutela, catalogazione che deve continuare e che, sempre più, dovrà fornire dati di qualità per il web, a partire dalla condivisione di authority file e thesauri. ${ }^{7}$

Lo sviluppo dell'informatica e il caricamento online delle collezioni visualizzabili come oggetto digitale, ascoltabili se si tratta di documenti sonori, stanno determinando trasformazioni continue nelle modalità di fruizione dei beni culturali. Anche l'allargamento del pubblico che frequenta i luoghi della cultura, come dimostrano i dati di crescita del turismo culturale, è un fattore importante. Basti pensare che ormai i dispositivi elettronici stanno sostituendo la classica guida cartacea sia per la fruizione remota, ad esempio quando si programma una visita, che per la fruizione in sede, dove curiosità e approfondimenti possono trovare una risposta immediata nel web.

7 Alla base della tutela del patrimonio sta appunto la catalogazione, considerata come la fase conoscitiva imprescindibile per la corretta gestione e conservazione dei beni. Per quanto riguarda la catalogazione, l'obbligo di legge è individuato dal Codice dei beni culturali e del paesaggio (D. Lgs 42/2004 e s.m.i. all'art. 17). A proposito della valorizzazione del patrimonio culturale attraverso la rete, e dell'importanza assunta dal tema dei dati collegati fra loro da relazioni qualificate che ne precisano il ruolo, perché essi creano le premesse per un accesso unificato e globale alle risorse culturali, vedi anche Bellia, Sabba 2017. 


\section{Mostre e nuova conoscenza}

Nonostante il titolo di questo seminario, desidero comunque riportare l'attenzione anche su un'accezione più ampia dell'azione di mostrare/mettere in mostra, intendendo le esposizioni documentarie organizzate direttamente dall'istituto conservatore come un ulteriore strumento per l'accesso al patrimonio; per dare conto con efficacia del lavoro (catalogazione, interventi conservativi, ricerca) compiuto sui beni 'mostrati'; per comunicare il valore di materiali che, entrati nelle raccolte, hanno assunto una speciale vocazione e per raggiungere così l'obiettivo di metterli a disposizione del pubblico più vasto attraverso occasioni di comunicazione narrativa (attività didattica, laboratori, incontri, ma anche mostre reali e virtuali) che più facilmente innescano processi di nuova conoscenza. ${ }^{8}$ Tornando al concetto che valorizzare significa anche favorire una fruizione sociale o allargata dei beni bibliografici e documentari, penso che le mostre siano una modalità da tenere in considerazione. Se accompagnate da adeguati strumenti didattici e divulgativi, costituiscono, infatti, occasioni importanti per avvicinare le persone al patrimonio documentario e promuovere processi di 'riappropriazione' pubblica dei beni culturali e di sensibilizzazione collettiva circa i problemi di tutela e conservazione. Le mostre ottengono generalmente un buon successo di visitatori perché realizzano una maggiore incisività comunicativa presso il pubblico, anche quello generico e meno preparato. Questo accade, in primo luogo, perché esporre è raccontare: il tema affrontato è illustrato in modo coerente, attraverso un percorso espositivo e un apparato didattico che devono essere progettati per mettere in risalto le relazioni fra i documenti e per chiarire il contesto all'interno del quale sono stati prodotti, ad esempio esponendo altre testimonianze utili a illustrare il periodo, il fenomeno, il personaggio collegati al tema della mostra. Tornando ai fondi d'autore, le esposizioni attraggono chi vuole soddisfare qualche curiosità sull'autore (ad esempio sulla sua vita, le amicizie, ecc.) o approfondire la conoscenza della sua opera. Con l'aiuto di materiali originali è più facile sforzarsi di penetrare il cuore dell'opera, avvicinarsi il più possibile all'autore e al suo contesto, diversamente da quanto è dato di fare leggendo un saggio. Si sperimenta la sensazione storica, cioè la sensazione del contatto immediato col passato (Korteweg 2009, 28). Gli oggetti, le testimonianze materiali selezionate per queste esposizioni - che possono essere mostre dossier temporanee oppure esposizioni permanenti con rotazione dei pezzi - assumono un forte potere evocativo, immaginativo o emotivo, diventando portatori di forma e contenuto di narrazioni che creano uno spazio empatico tra il manu-

8 Ho trattato per la prima volta questi argomenti nel 1994: Manfron 1994. 
fatto (reperto originario e reliquia biografica) e chi lo osserva. Sta poi nelle capacità dei curatori della mostra far sì che coerenza del tema trattato, contestualizzazione, allestimento e percorso espositivo determinino nel visitatore una felice combinazione fra l'esperienza visiva e i contenuti concettuali, magari da approfondire in un momento successivo grazie alla versione virtuale della mostra - dove è possibile caricare la riproduzione integrale dei pezzi selezionati - o incontri a tema e visite guidate dai bibliotecari e dai curatori, i quali potranno così direttamente trasmettere modalità e contenuti del proprio lavoro e favorire, finalmente, l'accesso diretto in biblioteca agli originali in quanto beni comuni e patrimonio di tutti e per tutti. ${ }^{9}$

\section{Bibliografia}

Bellia, A.; Sabba, F. (2017). «Modelli per la comunicazione dei dati di ricerca in archeologia: il caso dei Getty vocabularies come linked open data». AlB Studi, 57(1), 97-100.

Bianchini, C. (2015). «Dai cataloghi alla navigazione semantica». Guerrini, M.; Possemato, T. (a cura di), Linked data per biblioteche, archivi e musei. Perché l'informazione sia del web e non solo nel web. Milano: Bibliografica, 173-209.

Bruni, S. et al. (2016). «Verso l'integrazione tra archivi, biblioteche e musei. Alcune riflessioni». JLIS.it, 7(1), 225-44.

Carandini, A. (2017). La forza del contesto. Roma-Bari: Laterza.

Crocetti, L. (1999). «Memorie generali e memorie specifiche». Biblioteche Oggi, 4, 24-7.

Crocetti, L. (2001). «Parole introduttive». Messina, M.; Zagra, G. (a cura di), Conservare il Novecento $=$ Atti del Convegno nazionale (Ferrara, 25-26 marzo 2000). Roma: Associazione Italiana Biblioteche, 23-6.

Desideri, L.; Calabri, M.C. (2009). «Collezioni speciali del Novecento. Le biblioteche d'autore: definizione e gestione». Collezioni speciali del Novecento. Le biblioteche d'autore =Atti della Giornata di studio (Firenze, 21 maggio 2008). Firenze: Polistampa, 155-7.

Faggiolani, C. (2019). «Una svolta narrativa per la biblioteconomia italiana?». Faggiolani, C., Conoscere gli utenti per comunicare la biblioteca. Il potere delle parole per misurare l'impatto. Con contributi di Maddalena Battaglia et al. Milano: Bibliografica, 35-79.

Ferrer, D. (2001). «Introduction. Un imperceptible trait de gomme de tragacanthe». D'lorio, P.; Ferrer, D. (sous la direction de), Bibliotheques d'ecrivains. Paris: CNRS, 7-27.

9 Ho già trattato questi temi in Manfron 2012b, 2019. Interessante la definizione di «mostre necessarie» come «quelle capaci di mettere in contatto ricerca e grande pubblico e di ridare agli italiani le chiavi estetiche, etiche e civili del loro patrimonio» così come altre riflessioni a favore della valorizzazione finalizzata all'aumento della cultura e non alla spettacolarizzazione del patrimonio in mostre blockbuster «acchiappaturisti-mordi-e-fuggi» con mere finalità economico-commerciali in Montanari, Trione 2017. 
IFLA, International Federation of Library Associations and Institutions (2016). Dichiarazione di principi internazionali di catalogazione. https://www. ifla.org/files/assets/cataloguing/icp/icp_2016-it.pdf.

Korteweg, A. (2009). «Esposizioni letterarie: dove i lettori devono guardare». Kahars, A.; Gregorio, M. (a cura di), Esporre la letteratura. Percorsi, pratiche, prospettive. Bologna: Clueb, 23-41.

MAB (Musei Archivi e Biblioteche). Atto costitutivo. https://www. aib.it/wpcontent/uploads/2012/06/MAB20120612.pdf.

Manfron, A. (1994). «La biblioteca si mostra: problemi dell'esibire libri». Bollettino AIB, 3, 291-300.

Manfron, A. (2011). «Biblioteche e archivi d'autore: le relazioni da preservare». Antonelli, A. (a cura di), Spigolature d'archivio. Contributi di archivistica e storia del progetto "Una città per gli archivi". Bologna: Bononia University Press, 323-43.

Manfron, A. (2012a). «Biblioteca e archivio di persona: da fondo speciale a complesso documentario». Ghersetti, F.; Paro, L. (a cura di), Archivi di persona del Novecento. Guida alla sopravvivenza di autori, documenti e addetti ai lavori. Treviso: Fondazione Benetton Studi Ricerche; Fondazione Giuseppe Mazzotti; Antiga, 39-49.

Manfron, A. (2012b). «In studium non in spectaculum. Perché fare mostre in biblioteca». Zagra, G. (a cura di), Conservare il Novecento. Carte e libri in vetrina = Atti del Convegno (Ferrara, 1 aprile 2011). Roma: Associazione Italiana Biblioteche, 37-46.

Manfron, A. (2017). «Catalogare per esporre». Guarino, M.; Fabbri, I. (a cura di), IBC dossier. In primo piano. Libri, spartiti, documenti, lettere nei musei di scrittori e musicisti. Bologna: Istituto per i beni artistici, culturali e naturali della Regione Emilia Romagna, 6-10.

Manfron, A. (2019). «L'Archiginnasio: una biblioteca con vocazione museale». De Pasquale, A. (coordinamento scientifico di); De Capua, S. (a cura di), Le Biblioteche anche come Musei: dal Rinascimento ad oggi = Atti del Convegno internazionale (Roma, 16-17 novembre 2016). Roma: Biblioteca Nazionale Centrale di Roma, 259-67.

Manifesto IFLA/UNESCO sulle biblioteche pubbliche (1995). Trad. di M.T. Natale, AIB Notizie, 7(5), 1-2. http://www.aib.it/aib/commiss/cnbp/unesco.htm.

Montanari, T. (2018). Costituzione italiana: articolo 9. Roma: Carocci.

Montanari, T.; Trione, V. (2017). Contro le mostre. Torino: Einaudi.

Pisauri, N. (1988). «Lussuria e devozione». IBC informazioni, 4(3-4), 13-21.

Sangalli, F. (2016). Il sindacalista e Galileo. Miglioramento e capacità realizzativa nella nuova prassi sindacale. Milano: FrancoAngeli.

Zagra, G. (2007). «027.1 Biblioteche d'autore». Guerrini, M. (diretta da), Biblioteconomia. Guida classificata. Milano: Bibliografica, 719-20. 


\title{
Oltre le mostre
}

a cura di Mauro Brunello, Valentina De Martino e Maria Speranza Storace

\section{Archiporto: dalle carte d'archivio alle carte da gioco}

\author{
Sofia Stefani \\ Progetto Archiporto-Associazione Villa Fabris, Thiene (VI), Italia \\ Elisa Di Liberato \\ Centrale Fles/Fies Core, Dro (TN), Italia
}

\begin{abstract}
The Porto family's private archive held at the Castle of Thiene (Vicenza) is also a... castle of cards. More than 18,000 documents (XI-XIX centuries) transmit memories of words, lives and events which establish the history of a vast territory and its community. In addition to the inventory, preservation and digitalization of this heritage, the interdisciplinary Archiporto project includes among its objectives the identification of new strategies to communicate it to a wide audience: themes, stories and characters that emerge from archival records and become the protagonists of a game and a digital platform.
\end{abstract}

Keywords Archiporto project. Castle of Thiene. Porto-Colleoni-Thiene archives. da Porto family. Medieval and Early Modern times in Vicenza. Vicenza archives.

Sommario 1 Le carte dell'Archivio da Porto. - 2 Dalle carte d'archivio alle carte da gioco. 


\section{Le carte dell'Archivio da Porto}

«Ha voglia, Perotti», diceva, «di spendere per questa specie di penoso rottame tanto tempo e tanto sugo di gomiti! No, da' retta a me: qui, in questa semioscurità, uno può anche mettersi a gridare il miracolo, ma fuori, alla luce naturale, non c'è niente da fare, infinite magagnette saltano subito all'occhio, la vernice qua e là è partita, i raggi e i mozzi delle ruote sono tutti un tarlo, il panno di questo sedile [...] è ridotto in certi punti a una tela di ragno. Per cui mi domando: a che scopo tutta la struma di Perotti? Ne vale la pena? [...] Anche le cose muoiono, caro mio. E dunque, se anche loro devono morire, tant'è, meglio lasciarle andare. C'è molto più stile, oltre tutto, ti sembra?»

Micòl, colta e sfuggente protagonista del romanzo Il giardino dei Fin$z i$-Contini di Giorgio Bassani, trasforma la memoria in uno stile, ritenendo i ricordi legati a un oggetto, a un fatto o a una persona di gran lunga superiori al loro reale possesso nel tempo presente.

«Per lei avere stile vuol dire guardare al futuro. Eppure nella vita colleziona oggetti del passato» (Longo 2012, 265), quei làttimi - i vetri di Murano - ricercati per ore e ore tra gli antiquari veneziani, anche se per loro natura inevitabilmente destinati al deperimento e all'oblio. Nel racconto si profila un mondo in bilico tra passato e futuro, proteso verso la ricerca di un elemento d'unione capace di arrestare l'inarrestabile progresso del tempo, permettendo agli eventi e alle cose di entrare a far parte della coscienza collettiva. Ne deriva una riflessione tutt'oggi attuale, soprattutto se rapportata al tema affrontato in questa sede: come trasmettere le memorie (materiali) di persone e i beni conservati in un archivio? Come coinvolgere un territorio, e in particolare le nuove generazioni, nella riscoperta delle proprie radici senza incorrere in una divulgazione rivolta, compresa o ricercata soltanto da un pubblico di specialisti?

Fin dai tempi antichi la letteratura, il teatro, la musica, la pittura e tutte le molteplici manifestazioni dell'arte contribuiscono alla diffusione dei pensieri, degli avvenimenti e delle storie sia generali che individuali, imprescindibili per la creazione dell'identità storica, economica e sociale di ogni singolo individuo. Scegliere di diffondere una memoria attraverso produzioni intellettuali, siano esse relative a fatti realmente accaduti o frutto della fantasia, legate a personaggi, oggetti o semplici emozioni, equivale a tenere in vita l'essenza stes-

Il contributo è frutto della stretta collaborazione tra le Autrici. Per quanto riguarda la stesura materiale, Sofia Stefani è responsabile del § 1 «Le carte dell'Archivio da Porto», ed Elisa Di Liberato del § 2 «Dalle carte d’archivio alle carte da gioco». 
sa delle cose e, non meno importante, ad arricchire il bagaglio culturale della nostra storia collettiva.

Già Alessandra Cianelli associava la perdita della memoria a uno dei terrori più antichi dell'umanità: l'assenza di informazioni sul nostro passato equivarrebbe a perdere una solida e riconosciuta identità, oggi messa sempre più a rischio dal progresso apportato dalla rivoluzione informatica. Se da un lato, infatti, vi è una maggiore facilità nel reperimento e sviluppo dei dati (si pensi, per esempio, al campo della fotografia), dall'altro ci si imbatte sempre più di frequente nella mancata sedimentazione fisica dello stesso materiale, esposto al pericolo di una rapida dissoluzione. ${ }^{1}$

Il cambiamento nelle pratiche di ricerca, conservazione e valorizzazione delle informazioni sta modificando la funzione socio-culturale delle fonti, ivi comprese quelle conservate in biblioteche e archivi: la maggior parte delle strategie attualmente esistenti fanno del web il nuovo immenso archivio della contemporaneità, nonché il principale motore di ricerca, relegando in secondo piano la consultazione fisica di libri e documenti, oramai sempre più associati ad attività e indagini di studiosi, specialisti o semplici appassionati di specifici settori scientifici. Gli archivi possono però rappresentare il futuro della memoria, cercando di configurarsi consapevolmente come un mezzo di comunicazione attivo e in linea con la velocità richiesta dal mondo digitale, soddisfacendo il bisogno di servizi culturali proveniente da pubblici sempre più diversi e che necessitano di informazioni a livelli differenti. ${ }^{2}$ Con questo principio è nato il Progetto Archiporto, finalizzato alla conoscenza, conservazione, valorizzazione e messa in rete dell'Archivio privato della famiglia da Porto conservato a Thiene, nel vicentino, in una delle antiche dimore del casato: il quattrocentesco Castello Porto-Colleoni-Thiene. ${ }^{3}$

Il progetto, supportato dalla Fondazione Cariverona e attualmente ancora in corso (2018-21), vede coinvolto un gruppo di diversi enti e professionisti del settore storico-artistico, della conservazione

1 Il riferimento in: http://www.technoculture.it/author/a-cianelli/. Cf. anche Brunetta, Zotti Minici 2014.

2 Si veda, a questo proposito, il convegno promosso dagli Archivi di Stato nell'ormai lontano 1991 (Il futuro della memoria 1997). Rimando inoltre agli studi di Isabella Zanni Rosiello raccolti in Bianchi, Di Zio 2000.

3 Più comunemente chiamato Castello di Thiene. L'edificio, molto probabilmente risalente alla metà del XV secolo, costituisce un unicum per caratteristiche architettoniche e funzioni svolte, divenendo un caposaldo nell'evoluzione delle ville venete grazie al suo ruolo di dimora residenziale e fondiaria, a metà via tra il palazzo-fortezza di concezione medievale e la casa-fondaco veneziana; luogo di interessi economici ma anche culturali, entrambi prerequisiti della villa palladiana. Nel corso dell'Ottocento la proprietà passò ai Colleoni e, in seguito, ai conti di Thiene, tutt'oggi proprietari del castello. Per approfondimenti si veda, in particolare: http://www.castellodithiene. com; Morresi 1988; di Thiene 1995, 2005. 
del patrimonio archivistico e bibliografico e della comunicazione dei prodotti culturali, riuniti nell'obiettivo di fornire una nuova proposta di rete territoriale. ${ }^{4}$ Le differenti competenze messe in campo hanno permesso una visione più ampia trasformando l'eterogeneità del gruppo in un punto di forza: attraverso il dialogo continuo tra figure legate alla ricerca scientifica (archivisti, storici, specialisti in conservazione del patrimonio archivistico e librario e paleografi) e quelle impegnate nello sviluppo di prodotti e strategie innovative nel campo storico-artistico (performance art, biodiversità culturali, pratiche transdisciplinari), si è cercato di individuare criticità, differenze, problematiche, ma anche tematiche comuni, curiosità e opportunità da mettere in gioco per una maggiore fruizione dell'Archivio Porto.

Il lavoro è stato articolato in diverse fasi ${ }^{5}$ necessarie per l'analisi degli oltre 18.000 documenti di cui è composto il fondo, a sua volta correlato con gli archivi Colleoni, Capra e Thiene presenti al Castello. ${ }^{6}$ Il corpus documentario, già ordinato nel corso del XVIII secolo a seguito di questioni ereditarie, comprende pergamene, mappe e disegni, lettere, bolle papali e ducali, privilegi imperiali, libri di conti e vari altri materiali, originali e in copia, prodotti nei secoli XI-XIX.

Nella prima fase di censimento sono stati digitalizzati gli otto catastici di corredo redatti nel corso del secolo XVIII, funzionali sia alla compilazione di un nuovo inventario digitale, sia a tutelare e rendere più veloce la loro fruizione da parte degli utenti che, in questo modo, potranno consultarli virtualmente senza recarsi fisicamente in sede. I dati inseriti nell'inventario, pensato per essere caricato in rete, sono stati scelti per rilevanza ed efficacia nel campo della ricerca: per ogni documento - o in alcuni casi per la singola unità archivistica - è stata indicata la data, il numero di riferimento o il titolo, la tipologia (atto di vendita o di acquisto, testamento, ecc.), il nome del notaio e l'indicazione del luogo in forma di voce d'indice. La verifica dei materiali in Archivio ha permesso di individuare molteplici

4 Archiporto è formato da: Associazione Villa Fabris (ente capofila), Castello di Thiene, Biblioteca civica Bertoliana, Università Iuav di Venezia - MeLa Media Lab, Centrale Fies; in collaborazione con Dipartimento di Scienze Storiche, Geografiche e dell'Antichità - DiSSGeA dell'Università degli Studi di Padova e con il contributo di Melania Zanetti e Carlo Federici, per il progetto di conservazione del patrimonio archivistico, di Andrea Savio (consulente storico), Leonardo Granata (paleografo) e della scrivente (archivista). Questo studio raccoglie riflessioni e proposte avanzate durante il lavoro di gruppo: si ringraziano a questo proposito Giovanni Giuliari e Stefania Barsoni; Francesca e Giacomo di Thiene; Mattea Gazzola; Malvina Borgherini; Virginia Sommadossi, Luca Melchionna, Elisa Di Liberato e Lorenzo Facchinelli.

5 Digitalizzazione e censimento; studio e conoscenza; tutela e conservazione; comunicazione e valorizzazione; messa in rete e ricadute sul territorio.

6 L'Archivio, vincolato dal 1972 e dichiarato d'interesse regionale nel 2006, raccoglie anche numerosi frammenti di archivi diversi relativi alle proprietà acquisite per dote o per lascito testamentario. Il complesso archivistico è in parte inventariato ed è stato oggetto di interventi di restauro e conservazione. 
aspetti non riportati nella descrizione dei catastici, come la presenza di oltre cento mappe e disegni non indicati, errori di datazione o di numerazione, documenti mancanti, estrapolati, redatti in copia o danneggiati. ${ }^{7}$ Proprio per garantire una migliore salvaguardia di una parte dell'Archivio, si è svolta una Summer School di aggiornameni to in conservazione del patrimonio archivistico articolata in lezioni teoriche e pratiche, destinata a giovani e specialisti del settore del restauro. ${ }^{8}$ Durante l'attività di laboratorio i partecipanti hanno realizzato le analisi e gli interventi su alcuni documenti membranacei e su alcune mappe del territorio vicentino.

La compilazione del nuovo inventario ha consentito inoltre di accertare la presenza di numerosi documenti inediti relativi ad attività economiche del passato, a personaggi, avvenimenti e fatti storici di particolare interesse. Questo aspetto ha incoraggiato il gruppo nell'identificazione di tematiche e argomenti utili per lo studio e l'approfondimento di alcuni fattori sociali e in primo luogo dell'aristocrazia vicentina, capace di emergere nella gestione di imprese agricole, commerciali e proto-industriali che si estendevano, in alcuni casi, oltre i confini del territorio nazionale. ${ }^{9}$ Tra questi sono stati selezionati i temi della gestione delle acque e dei mulini nel vicentino; dello sviluppo e affermazione delle antiche attività artigianali, come la produzione e il commercio della seta e altri tessuti, l'estrazione della cosiddetta 'terra bianca' (caolino), gli acquisti e vendite di pietre preziose e altri beni di lusso. Sono stati poi individuati i principali condottieri, ambasciatori e personaggi illustri appartenenti o in contatto con i da Porto in relazione con gli avvenimenti storici, politici, religiosi e culturali che li coinvolsero, come i rapporti intessuti dal casato con alcune corti italiane ed europee o le vicissitudini legate al periodo della Lega di Cambrai e della Riforma protestante, ancora bisognose di approfondimenti. Non meno importante il ruolo delle donne imprenditrici capaci di distinguersi in momenti storici

7 Il lavoro di descrizione inventariale informatizzata e di controllo dei materiali presenti in Archivio è a cura della scrivente con il coordinamento della Biblioteca civica Bertoliana (Mattea Gazzola). Affidata a M.I.D.A Informatica s.r.l., invece, la digitalizzazione dei catastici.

8 Il corso si è svolto durante l'estate 2019 nella sede del Centro Europeo per i Mestieri del Patrimonio ed è stato coordinato dall'Associazione Villa Fabris di Thiene. Le lezioni, articolate nel corso di quattro settimane intensive, sono state promosse in collaborazione con il corso di Laurea Magistrale in Storia e gestione del patrimonio archivistico e bibliografico del Dipartimento di Studi Umanistici dell’Università Ca' Foscari di Venezia (Melania Zanetti e Carlo Federici), coinvolgendo docenti universitari ed esperti della conservazione del patrimonio archivistico e librario.

9 I temi sono stati definiti sulla base delle conferme riscontrate in Archivio riguardo ad alcuni ambiti ritenuti di fondamentale interesse storico, economico, politico, religioso e culturale, individuati dal gruppo durante la fase progettuale con la supervisione di Andrea Savio. 
non sempre a loro favorevoli, testimonianza della ricchezza e affermazione di alcune famiglie vicentine - soprattutto nel corso dei secoli XV-XVI -, tra cui i Chiericati, i Valmarana, i Trissino, i Thiene, i Capra e molte altre. Di queste sono stati segnalati gli aspetti pertinenti ai contesti palladiani e alle principali case, palazzi dominicali e alle opere d'arte individuate all'interno degli inventari, divisioni dei beni, lasciti testamentari, donazioni e altre tipologie documentarie. Indirettamente, inoltre, molteplici atti e processi dell'Archivio Porto rendono testimonianze fondamentali, relativamente ad alcuni secoli e in specifiche zone (in particolare i paesi di provincia), per lo studio delle vicende agricole e della società contadina. I temi individuati con il progetto, uniti alle oltre cinquecento mappe e disegni conservati al Castello, possono offrire spunti per intraprendere nuove strade di ricerca: dal dialogo fra i diversi archivi ed enti del territorio al coinvolgimento delle scuole, fino ad arrivare a sensibilizzare la classe politica e imprenditoriale. Per interagire con la comunità, fornendo prodotti in grado di essere compresi e utilizzati da un ampio numero di persone, occorre però, in primo luogo, aprirsi a nuove strategie individuando per ogni target una specifica modalità di relazione. Le classiche proposte a cui, per tradizione e metodologia, ricorre il patrimonio archivistico per mostrarsi alla collettività (visite guidate negli archivi, mostre, edizioni di studi e ricerche, incontri e seminari), dovrebbero sempre più essere affiancate da percorsi trasversali adatti a scuole, università o a semplici appassionati, permettendo così di garantire un servizio culturale per il territorio di appartenenza. In quest'ottica si è scelto di mettere in relazione le carte dell'Archivio Porto con quelle degli altri archivi presenti nel vicentino attraverso un'innovativa piattaforma web: il nuovo portale degli archivi della Biblioteca civica Bertoliana di Vicenza.

Per migliorare la fruizione dei propri archivi e per ricercare strategie comuni, la Biblioteca Bertoliana ha da poco caricato online il proprio patrimonio archivistico, per la gran parte informatizzato, offrendosi di coordinare progetti di inventariazione di archivi di altri enti del vicentino, interessati a far parte di una rete virtuale di cooperazione.

Nell'ambito dell'iniziativa anche l'Archivio Porto, in collaborazione con la Soprintendenza archivistica e bibliografica del Veneto e del Trentino Alto Adige, ha messo a disposizione il proprio inventario digitale per aprirsi a un ampio pubblico, per fornire la possibilità di una ricerca più ricca in termini di informazioni e contenuti e per mettere in evidenza i nessi che legano i beni culturali di un territorio, indipendentemente dalla loro natura e dai luoghi in cui sono conservati. ${ }^{10}$

10 Parte delle informazioni sono tratte dal portale della Biblioteca Bertoliana consultabile al link: http://bertoliana.xdams.org/bertoliana/. Il progetto di pubblicazio- 
In questo senso, i temi individuati con il progetto Archiporto, oltre a consentire lo sviluppo di ricerche scientifiche a cura del gruppo di lavoro e di tutti coloro che vorranno approfondire tali argomenti, costituiranno la base di soluzioni interattive adatte a ragazzi di scuole, università e centri culturali. Il mondo dell'archivistica e degli operatori di settore, per far evolvere gli archivi permettendo loro di entrare a far parte dell'identità collettiva, dovranno scardinare sempre più le tipologie classiche di divulgazione per integrarsi e collegarsi con la contemporaneità attraverso l'uso, per esempio, dei social media, di giochi e percorsi interattivi. Solo così, forse, si potrà guardare al futuro, intraprendendo nuovi percorsi più efficaci per far rivivere e riscoprire gli archivi.

\section{Dalle carte d'archivio alle carte da gioco}

La necessità di individuare strategie comunicative straordinarie si è resa evidente fin dai primi incontri del gruppo di lavoro Archiporto. A partire dalla volontà di rendere disponibile al pubblico il fondo di un archivio privato - con la collaborazione dei professionisti di un'associazione culturale, di una biblioteca pubblica e di un media lab universitario, nonché di esperti in storia, archivistica, paleografia e conservazione del patrimonio archivistico e librario - ci si è chiesti quale fosse la formula più adatta per narrare storie di famiglie e di luoghi, emblemi del potere di un'élite nel passato, elementi d'aggregazione per la comunità nel presente.

Assunta come sfida l'idea che per diffondere le carte di un archivio occorresse sparigliarle, che le storie tratte dai documenti cartacei avrebbero avuto maggior respiro se incarnate in personaggi, organizzate secondo una drammaturgia e fatte conoscere alle diverse comunità del territorio, si è convenuto di aggregare al gruppo e di far curare la fase comunicativa del progetto a Fies Core, dipartimento del centro di produzione di arti performative Centrale Fies, specializzata nell'ideazione di progetti e sviluppo di strategie culturebased per il settore pubblico e privato.

L'operazione di divulgazione e comunicazione del progetto Archiporto si è a questo punto articolata in diverse azioni: analisi dello stato dell'arte, audit, ideazione e produzione di uno strumento utile per far conoscere l’Archivio Porto a un pubblico di non specialisti

ne dei dati, descritti con xDams Platform, è a cura di Regesta.exe. L'inventario dell'Archivio Porto è attualmente ancora in fase di lavorazione e sarà consultabile dal 2021 nella sezione Archivi di altri enti e istituzioni, in cui sono già a disposizione le schede relative al fondo e al soggetto produttore. 
e in grado di proporre una riflessione (o almeno un tassello della riflessione collettiva in atto) sull'importanza degli archivi nella cultura contemporanea.

Per affrontare tutte le singole fasi del processo che avrebbe in seguito portato al gioco di carte Archiporto, Fies Core ha creato un'équipe con competenze tali da elaborare una proposta generativa nel tempo: professionisti esperti di comunicazione, intesa sia come strategia che come linguaggio, di progettazione di ambienti digitali per musei, di arti performative, visive e design, di ricerca in ambito culturale, attivi nella creazione di progetti con un forte impianto partecipativo e orientati al coinvolgimento del territorio.

Lorenzo Facchinelli ha aperto la fase di analisi con una ricognizione dal titolo Archivio e Territorio. ${ }^{11}$ Fine della ricerca non è stato solo individuare pratiche particolarmente interessanti che potessero ispirare il progetto ma anche operare una riflessione sulle possibilità e le potenzialità di relazione tra archivi e territori. Dalla ricerca sono emersi due aspetti che hanno costituito in seguito la base di pensiero per il concept dello strumento di divulgazione dell'Archivio Porto: «il solo tema del 'territorio' non rappresenta un tema culturale, ma è necessario un rovesciamento di termini: non pensare di valorizzare il singolo archivio, ma il territorio grazie all'accesso all'archivio». E ancora,

bisogna considerare l'archivio alla stregua di altre risorse culturali di un territorio con cui collabora. Senza alcuna soluzione di continuità tra cultura alta e bassa, tra risorse umane o naturali, tra ambiti, solo così si costruiscono alleanze in grado di attrarre, nel loro insieme, target molto più estesi di quelli che un singolo archivio potrebbe fare, ma alle quali un archivio, con il suo portato di contenuti e temi, può offrire un contributo sostanziale per costruire nuove esperienze e storie.

Questa riflessione apre uno scenario d'azione molto più ampio: non si tratta solo di creare progetti attrattivi ma di favorire sinergie in grado di connettere tra loro realtà e conoscenze fuori da ogni gerarchia o specializzazione. E di farlo nel tempo. Attivare quindi proget-

11 Archivi e Territorio è una ricerca elaborata da Lorenzo Facchinelli specificamente per il progetto Archiporto e non destinata alla pubblicazione dove vengono analizzati dieci casi contemporanei nazionali e internazionali di apertura al pubblico, valorizzazione e divulgazione di archivi storici. Facchinelli con Malvina Borgherini ritornano sull'argomento nel loro L'archivio nell'era della internet culture, presentato al Convegno organizzato dal Dipartimento di Filosofia, Sociologia, Pedagogia e Psicologia applicata dell'Università degli Studi di Padova (Padova, Sala delle Edicole, Palazzo del Capitanio, 6 marzo 2019), dal titolo Archivi di famiglia. Perché conservarli e quale futuro. La registrazione audio dell'intervento è disponibile online: https://youtu.be/NJxL5p23TwU. 
ti in grado di espandersi, mutarsi in strumenti fuori dal loro contesto e in grado di rigenerarsi nel tempo. Pensare quindi una dimensione performativa dell'archivio che si intrecci con l'espressione performativa del territorio.

Altro aspetto emerso dalla ricerca è il pubblico che s'intende agganciare (per accompagnarlo in seguito verso territori inesplorati), identificato come quello del culture snacker. Peter Gorgels, internet manager al Rijksmuseum di Amsterdam, afferma che viviamo in una cultura in cui l'immagine ha un grande peso. Siamo bombardati ogni giorno da migliaia d'immagini, le persone pubblicano le loro foto sui social media, direttamente dallo smartphone. La manipolazione creativa delle immagini è diventata di uso comune, sia per fini dilettevoli che estetici. Questa è la nuova impostazione in cui è attivo il culture snacker. Lui o lei ama guardare le immagini e condividerle con amici e follower. Tutti sono (in un certo senso) dei consumatori di pillole di cultura e oggi è importante che i musei li raggiungano. ${ }^{12}$

Il lavoro preliminare dell'équipe, le analisi e le sperimentazioni attuate su precedenti progetti hanno portato all'identificazione del gioco come ideale strumento d'attrazione per la sua capacità di mettere in connessione differenti comunità e di costituirsi come esperienza di conoscenza appassionante, coinvolgente, replicabile. Ma se la gamification è ormai una pratica consolidata nei processi di audience engagement nel mondo culturale - in particolare nei musei e nelle istituzioni dedicate alla valorizzazione del patrimonio - l'individuazione delle più efficaci tecniche e tipi di esperienza da proporre si apre su un orizzonte di innumerevoli possibilità. E se il digitale ha assunto ormai un ruolo imprescindibile all'interno di questi processi, rimane aperta la domanda su come verrà esercitato quel ruolo, se lo spazio virtuale sarà quello sul quale l'intera esperienza si compirà o se agirá in interdipendenza con altri spazi e in momenti diversi. In coerenza con uno degli obiettivi del progetto Archiporto - ovvero costituirsi come risorsa culturale per il territorio - il gruppo ha condotto un'indagine sulle possibili comunità di riferimento ${ }^{13}$ per sondarne gli interessi relativi agli archivi storici, le preferenze sulle modalità di gioco e il tipo di esperienza alla quale avrebbero aderito con più entusiasmo. All'audit in forma di questionario hanno partecipato più di duecento persone e i risultati emersi hanno indicato con molta chiarezza il desiderio di conoscere le storie dei personaggi legati al castello e di scoprire il contenuto dei documenti, di poter avere un'esperienza di tipo esplorativo e di poterla vivere attraverso un gioco.

12 Cf. https://mw2013.museumsandtheweb.com/paper/rijksstudio-make-yourown-masterpiece/.

13 L'indagine, curata da Luca Melchionna, si è svolta presso Villa Fabris, Castello di Thiene, Biblioteca civica Bertoliana di Vicenza dal maggio 2018 all'ottobre 2019. 
Elisa Di Liberato, Sofia Stefani

Archiporto: dalle carte d'archivio alle carte da gioco
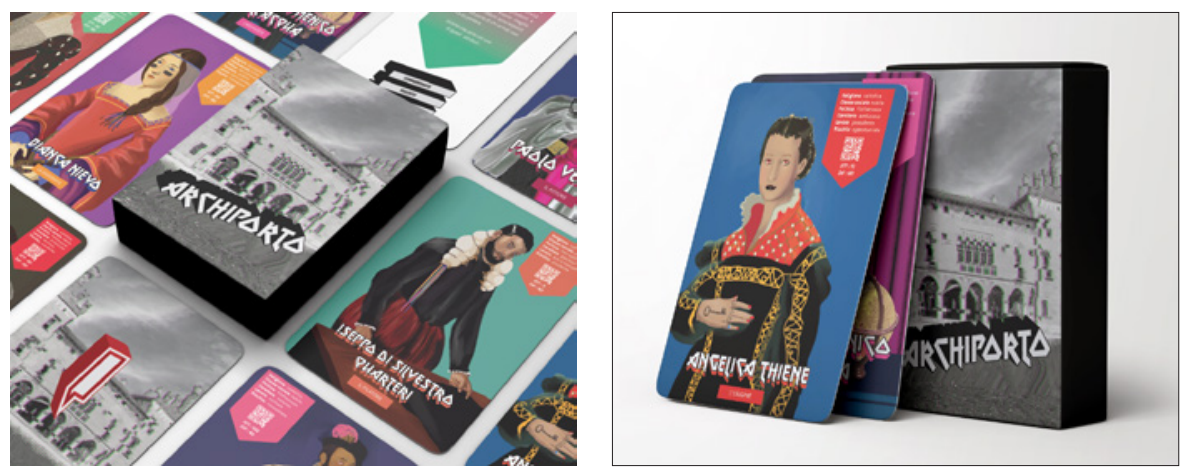

Figure 1-2 Le carte del gioco Archiporto con le illustrazioni di Giordano Poloni, la direzione artistica di Virginia Sommadossi e il game design di Machineria, dedicato alla valorizzazione dell'Archivio della famiglia da Porto conservato al Castello di Thiene (VI)
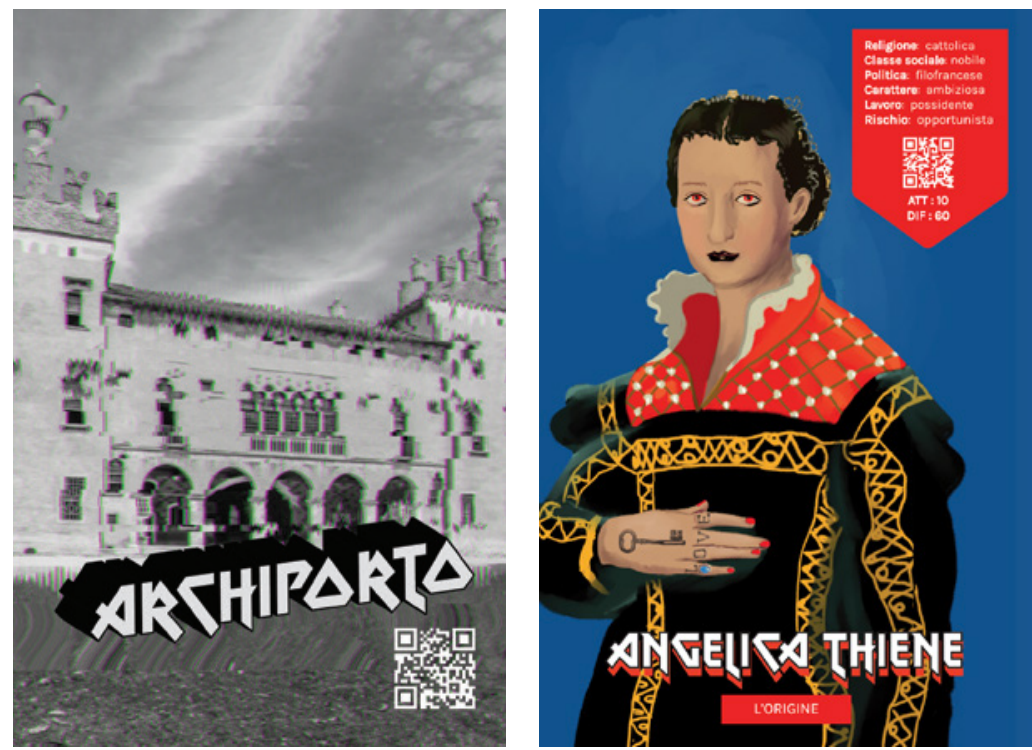

Figure 3-4 Recto e verso della carta di Angelica di Thiene, personaggio chiave nelle vicende legate alla tutela e conservazione dell'Archivio Porto. Il ritratto di Angelica Thiene è realizzato dall'illustratore Giordano Poloni 
Da queste evidenze, e attraverso un lavoro condiviso, nasce il gioco di carte Archiporto ${ }^{14}$ [figg. 1-4].

Il gioco è composto da quattordici carte, ognuna dedicata a un personaggio, e otto carte dedicate ad altrettanti eventi storici (denominati Imprevisti) della Vicenza del XVI secolo, utilizzabili in sei diverse dinamiche, alcune delle quali pensate specificamente per i giovanissimi e per le scuole. Ogni dinamica di gioco interpreta una diversa situazione, predisposizione e gruppo: si può giocare da soli o in due o più persone, o con il pieno coinvolgimento, a scuola, di una classe. Archiporto diventa così una trama di caratteri e vicende utile a stimolare il problem solving, così come l'attitudine al gioco di ruolo, oppure si pone come strumento attivatore di ricerche storiche di gruppo o di co-creazione di narrazioni. Un oggetto compatto nella forma, quindi, ma in grado di attivare molteplici possibilità e occasioni di incontro, confronto, esperienza e conoscenza.

Parte del gioco si svolge online: su ogni carta è presente un QR code che rimanda a una scheda di approfondimento relativa alla storia del personaggio cui si riferisce, le sue vicende, le sue attitudini e il contesto in cui ha vissuto. La scheda è inoltre arricchita da consigli per nuove letture. Si è inoltre ritenuto necessario stabilire delle connessioni narrative tra personaggi e giocatori attraverso alcuni elementi contemporanei affinché le storie del passato potessero risuonare nel presente e rendersi memorabili anche tra le comunità più giovani. Ecco allora un elemento per ciascun personaggio, un $\mathrm{Ma}$ gnifico Anacronismo (ispirato alla storia o al carattere del personaggio), che si comporta come un bug all'interno di un codice iconografico aderente a quello della ritrattistica dell'epoca: un tatuaggio old style sulla pelle (Angelica Thiene e Ludovico Porto), i pin Free Angela e il volto di Angela Davies appuntati sul petto (Maddalena Campiglia), l'eyeblack dei giocatori di football (Bianca Nievo), il volume Python for Dummies tra le mani (Laura Thiene), ecc.

Questa scelta ha cambiato radicalmente la natura delle illustrazioni: i dettagli contemporanei aggiunti hanno conferito alle storie un potenziale evocativo che le porta fuori da una dimensione individuale e temporale per condurle verso uno spazio di interesse collettivo e di rilievo nella contemporaneità - vi compaiono elementi che rimandano alla parità di genere e agli stereotipi, al rapporto tra arte contemporanea e artigianato, all'importanza degli archivi storici, alla possibile decodificazione dei simboli del potere nelle immagini - che contiene interessanti spunti di conversazione per mettere in contatto differenti generazioni e varie comunità.

14 Il gioco Archiporto è stato elaborato da Machineria e prodotto con la direzione artistica di Virginia Sommadossi, i testi di Luca Melchionna e le illustrazioni di Giordano Poloni; punto di partenza le indicazioni storiche fornite da Andrea Savio. 
Ė questa «continuità tra cultura alta e bassa» - citando ancora l'analisi di Facchinelli - che permette di far affiorare un quadro complessivo, denso e articolato, che non si esaurisce con un numero di conoscenze da acquisire. Un affresco storico prende forma sotto gli occhi dei giocatori carta dopo carta, dettaglio dopo dettaglio, in un continuo rimando tra passato e presente, tra personaggi ed eventi, tra personaggi e giocatori, tra dimensione individuale e collettiva.

Per favorire la diffusione del gioco e del progetto e per assicurare la massima accessibilità al pubblico, è stato realizzato un sito internet dedicato ${ }^{15}$ dal quale è possibile scaricare una versione delle carte stampabile per l'autoproduzione del mazzo. La proposta nel suo insieme risulta particolarmente efficace nei contesti scolastici anche fuori dal territorio di riferimento e rende possibile l'attivazione di una strategia a lungo termine per il rilancio istantaneo del gioco sui canali di comunicazione.

Allo scopo di far emergere in loco i personaggi delle carte (e soprattutto dar loro voce), si è pensato di attivare, tra le mura del Castello di Thiene - dove è ancora oggi conservato l'Archivio, soggetto / oggetto principale del progetto Archiporto - anche una presenza fisica, o meglio fantasmatica. A cura del MeLa Media Lab si è quindi avviato il progetto di un'installazione sonora (cui partecipano l'architetto e musicista Nicola Di Croce, la scrittrice Federica Iacobelli e l'attrice Mirella Matronardi) che avrà il suo momento di massima espressione tra gli stalli della scuderia settecentesca del Castello. Nelle stanze del Castello saranno inoltre predisposti dei diffusori acustici, che fungono da presentatori di personaggi fantasmi. I loro sussurri e le loro voci lontane fuoriescono dai legni e dalle pietre che rivestono lo spazio destinato nel passato all'animale emblema di libertà e parte insostituibile del personaggio condottiero.

Ancora una volta una presenza che prende corpo, non solo attraverso anacronismi e reinvenzione di immagini, ma anche attraverso la fisicità di materiali e suoni. Presenza che contribuisce a riattivare un mondo di carte, le scompagina, le fa rinascere. 


\section{Bibliografia}

Bianchi, C.; Di Zio, T. (a cura di) (2000). L'archivista sul confine. Scritti di Isabella Zanni Rosiello. Roma: Ministero per i beni e le attività culturali. Ufficio centrale per i beni archivistici.

Brunetta, G.P.; Zotti Minici, C.A. (a cura di) (2014). La fotografia come fonte di storia = Atti del convegno (Venezia, 4-6 ottobre 2012). Venezia: Istituto Veneto di Scienze, Lettere ed Arti.

Il futuro della memoria = Atti del convengo internazionale di studi sugli archivi di famiglie e di persone (Capri, 9-13 settembre 1991) (1997). Roma: Ministero per i beni culturali e ambientali. Ufficio centrale per i beni archivistici.

Longo, F. (2012). "Lettura retorica del "Giardino dei Finzi-Contini" di Giorgio Bassani». Antognini, R.; Diaconescu Blumenfeld, R. (a cura di), Poscritto a Giorgio Bassani. Saggi in memoria del decimo anniversario della morte. Milano: LED, 247-70.

Morresi, M. (1988). Villa Porto Colleoni a Thiene. Architettura e committenza nel Rinascimento vicentino. Milano: Electa.

di Thiene, C. (a cura di) (1995). Il castello Porto-Colleoni-Thiene. Trento: Odos.

di Thiene, C. (2005). «Villa Porto-Colleoni-Thiene a Thiene (Vicenza)». Beltramini, G.; Burns, H. (a cura di), Andrea Palladio e la villa veneta da Petrarca a Carlo Scarpa = Catalogo della mostra (Vicenza, Museo Palladio in palazzo Barbaran da Porto, 5 marzo-3 luglio 2005). Venezia: Marsilio, 258-61, schede nrr. 42 e $42 a$. 



\title{
L’alluvione del 1966 e la Biblioteca Nazionale Un 'safari' della memoria
}

Alessandro Sidoti

Biblioteca Nazionale Centrale e Opificio delle Pietre Dure, Firenze, Italia

\begin{abstract}
Pictures and stories from the 1966 flood at the National Central Library of Florence told in an interactive exploration. A visit to relive in the present, through augmented reality, the photographs and videos of the time observed from the same point of view of 1966, in a journey in steps that reconstructed the most important moments of the flood. The project also allowed the digitization of approximately 3,500 images of the National Library's photographic archive scanned at high resolution, filed in the Library's online catalog (OPAC).
\end{abstract}

Keywords Florence. Flood. Photographs. Virtual reality. Augmented reality. App.

Sommario 1 Introduzione. - 2 La città e l'alluvione. - 3 La Biblioteca Nazionale. 4 Come raccontare l'alluvione del 1966 e la Biblioteca Nazionale. - 5 Visite virtuali. 6 Il sito ARNO66ar.it. - 7 L'app ARNO66 TimeTravel. - 8 Digitalizzazione dell'archivio fotografico. - 9 II CEDAF.

\section{Introduzione}

Il progetto Safari della memoria è stato realizzato grazie a una collaborazione della Biblioteca Nazionale Centrale di Firenze (BNCF) con i creativi di Nanof, Lorenzo Garzella e Filippo Macelloni. ${ }^{1}$ Lo scopo del progetto era com-

1 Il progetto Safari della memoria è stato realizzato grazie a una collaborazione della BNCF con i creativi di Nanof, Lorenzo Garzella e Filippo Macelloni, Acquario della Memoria Associazione Culturale. Realizzato nell'ambito del Programma Sensi Contemporanei per il Cinema. Cataloga- 
plesso e ambizioso: cercare di riportare l'utenza indietro nel tempo al momento dell'alluvione, l'idea era di provare ad andare, appunto, 'oltre le mostre' ideate nel passato per ricordare il drammatico evento, cercando di uscire dalla ripetitiva, e ormai sterile, celebrazione dell'alluvione.

Il progetto realizzato in occasione del cinquantennale, si accostava comunque alla grande mostra cittadina La bellezza salvata (Acidini Luchinat, Capretti 2016) in cui sono stati esposti molte altre tipologie di beni, non solo della BNCF, in cui il materiale librario e archivistico finalmente era mostrato insieme alle altre opere d'arte. Quindi non più una mostra in archivio e biblioteca, ma un'esposizione che coinvolgeva tutti i beni colpiti. La BNCF voleva avvicinare le persone per far rivivere quei momenti, rivolgendosi ad un pubblico nuovo di visitatori diverso dall'utenza che normalmente frequenta la biblioteca.

\section{La città e l'alluvione}

Nella notte fra il 3 e il 4 novembre 1966, dopo giorni di pioggia incessante, l'acqua dell'Arno uscì dagli argini e travolse Firenze. Per 18 ore la città rimase in balia di un miscuglio furioso di fango e detriti di ogni tipo, a cui si aggiunse la nafta uscita dalle caldaie. Il livello dell'acqua raggiunse i 6 metri nei quartieri più colpiti e quando il fiume si ritirò restituì uno scenario apocalittico. Le vittime furono 35: 17 in città e 18 nei comuni limitrofi. I danni al patrimonio furono incalcolabili (Alessandri, Fontana 2006).

L'alluvione colse di sorpresa le autorità italiane e, dopo un primo sbandamento, la reazione fu formidabile.

Ai fiorentini, alle forze dell'ordine, all'esercito si sommarono centinaia di persone che giunsero spontaneamente da tutta Italia e dal mondo. Un amalgama mai visto prima di volontari, soprattutto giovani, che riuscì a trasformare l'emergenza in un momento di riscatto collettivo. Furono battezzati 'gli angeli del fango', fu uno dei primi esempi di mobilitazione giovanile spontanea del XX secolo.

Non chiedono nulla, vogliono salvare la cultura, vanno a spalare nei musei, nelle chiese, nelle biblioteche a salvare i capolavori, i libri, gli arredi sacri [...], si coprono di fango: fango della Biblio-

zione digitale dell'archivio fotografico: Carmelo Albanese, Antonio Iannello con la collaborazione di: Leandra Alessandrello, Gianmarco Orsi, Alice Maniaci. Programmazione informatica ARNO-66: Andrea Camporeale con la collaborazione di Luca Gronchi. Grafica e progettazione web: Net7. L'elaborazione della silhouette della facciata della biblioteca è di Serena Tonelli. 
teca Nazionale, dell'Archivio di Stato, della Biblioteca Viesseux, dei chiostri di Santa Croce, dei depositi degli Uffizzi [...], fango di ogni istituzione culturale, fango della Sinagoga, di San Frediano, fango di ogni quartiere. (Batini 1967, 30)

\section{$3 \quad$ La Biblioteca Nazionale}

Il 4 novembre 1966, alle 6:50 del mattino l'Arno in piena ruppe la prima spalletta di fronte alla Biblioteca Nazionale Centrale. In poche ore il pianterreno e i sotterranei furono invasi dalla melma.

Oltre un milione di volumi rimase danneggiato: 300.000 libri, 400.000 volumi tra giornali e quotidiani, 60.000 volumi di riviste, 400.000 pezzi di opuscoli ed estratti delle miscellanee (Guasti 2020).

Fra i libri rovinati si trovarono i testi antichi delle collezioni Magliabechiana e Palatina, inclusi i preziosi grandi formati conservati nel sottosuolo. Si salvarono invece i manoscritti e gli incunaboli, conservati al primo piano.

Nei giorni immediatamente successivi al disastro la Biblioteca Nazionale diventò il centro e il simbolo dei soccorsi prestati spontaneamente da giovani di tutti i paesi. Dall'ammasso di fango i libri vennero recuperati uno ad uno e passati di mano in mano attraverso catene umane di salvataggio.

$\mathrm{Fu} \mathrm{l'occasione} \mathrm{per} \mathrm{una} \mathrm{irripetibile} \mathrm{esperienza} \mathrm{di} \mathrm{asciugatura} \mathrm{e} \mathrm{re-}$ stauro, che permise di sperimentare tecniche diverse e - anche grazie all'aiuto di esperti internazionali - fondò nuovi criteri nel restauro librario, generando un laboratorio all'avanguardia nel mondo.

In Biblioteca Nazionale si restauravano libri anche prima dell'alluvione, si restauravano [...], così come credo nella totalità delle biblioteche italiane, senza nessun principio. Mancavano assolutamente alcuni principi generali che con l'alluvione sono stati ripristinati, come ad esempio, il principio generale che un libro è una struttura, [...], e si deve intervenire non restaurando questo o quel particolare, ma l'intera struttura. (Maini 1996, 21) 


\section{Come raccontare l'alluvione del 1966 e la Biblioteca Nazionale}

Il progetto è stato pensato per mantenere viva la memoria, con la preoccupazione che, con l'andare del tempo, sempre di più diventasse una celebrazione dell'evento del 4 novembre riservata solo al decennale e che tutto, in qualche maniera, andasse dimenticato. Nel quarantennale dell'alluvione, era emerso durante un convegno realizzato dall'Archivio di Stato di Firenze, ${ }^{2}$ come le nuove generazioni non percepissero più l'alluvione come quella che era stata veramente tanto che i bambini i disegnavano l'acqua che invadeva la città di colore celeste e non più marrone. La trasmissione di una memoria alle nuove generazioni era a rischio di perdita. Grazie alla ricchissima documentazione fotografica della Biblioteca è stato possibile mostrare immagini e raccontare storie dell'alluvione del 1966 in una esplorazione interattiva site specific attraverso un percorso con tablet e visori VR/AR ('cardboard' / tablet) che ha consentito di rivivere nel presente, tramite la realtà aumentata, le fotografie e i filmati dell'epoca.

Le più significative fotografie dell'archivio della BNCF sono state osservate dallo stesso punto di vista da cui sono state scattate nel 1966, in un percorso a tappe che ha ricostruito i momenti più importanti dell'alluvione: l'allarme, l'Arno che esondava, il portone che cedeva dopo lunga resistenza, il disastro, l'organizzazione del salvataggio, il lavoro degli 'angeli del fango', le catene umane per portare in salvo i volumi, le spedizioni ai forni per l'asciugatura dei libri, l'organizzazione del laboratorio di restauro di emergenza nel chiostro e poi nella sala lettura. Le visite sono state organizzate durante i consueti orari di apertura della biblioteca. Era necessario inoltre far convivere questo tipo di attività con una biblioteca di ricerca, che non è molto adatta a questa tipologia d'eventi che possono turbare la quiete delle sale di studio [figg. 1-3].

Lo scopo quindi era di riuscire a far entrare in biblioteca e rivivere nuovamente alcuni momenti, soddisfacendo anche il desiderio di chi spesso voleva rivedere i luoghi dove era stato, come volontario, cinquanta anni prima. Le generazioni passate insieme a quelle nuove erano il target destinato a favorire la trasmissione della memoria di quello che era effettivamente accaduto [fig. 4].

Il visore o il tablet dava la percezione di cosa effettivamente fosse avvenuto in precedenza in quel determinato luogo; la visita guidata accompagnava nei luoghi più significativi e consentiva di avere un'esperienza completa, provando ad immergersi nei vasti spazi della biblioteca, compresi i magazzini e, avendo la possibilità, con l'u-

2 http://www.archiviodistato.firenze.it/asfi/index.php?id=186. 


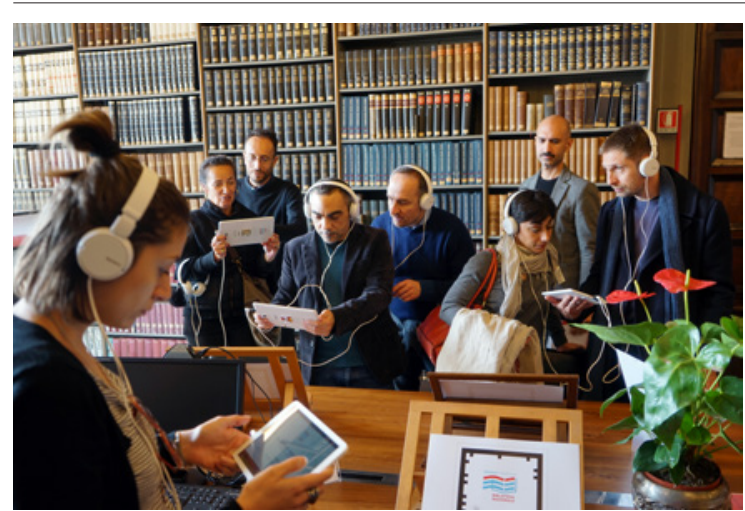

Figura 1 Visite guidate silenziose. Nanof, elaborazione grafica per concessione degli autori
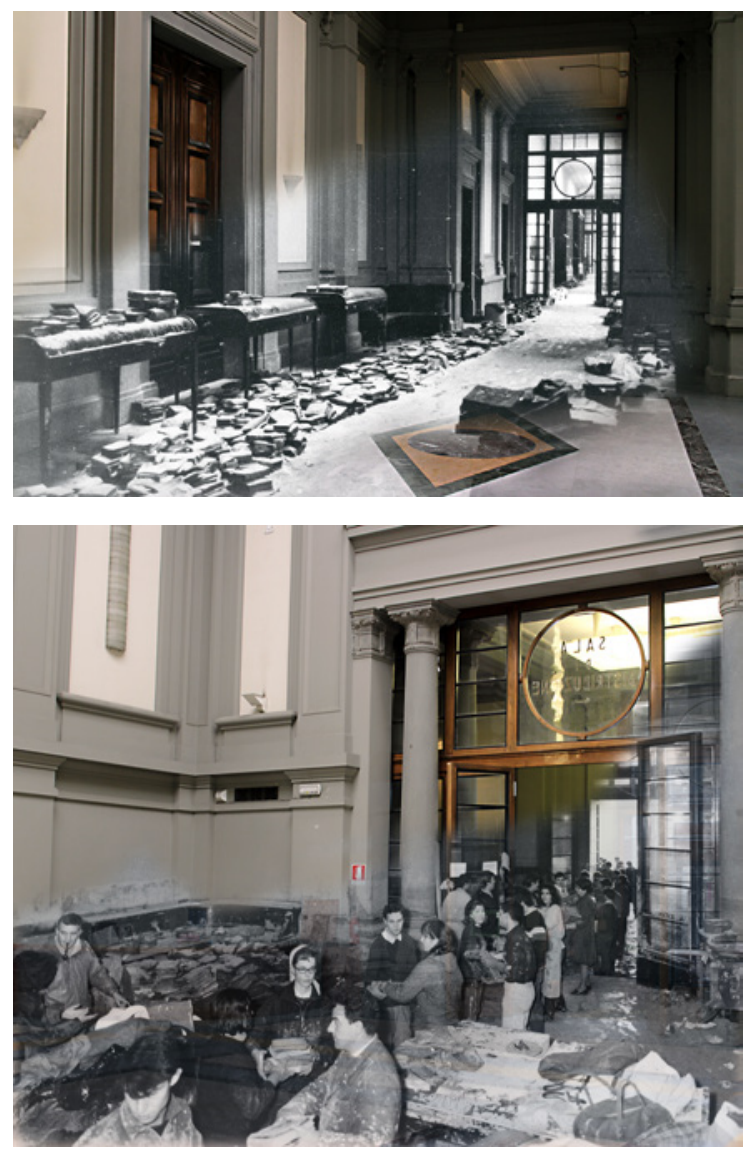

Figure 2-3 I locali a confronto. Nanof, elaborazione grafica per concessione degli autori 
so dei tablet in questo caso, di visualizzare il presente e il passato in contemporanea, con una comparazione dallo stesso punto di vista. Nei punti stabiliti giocando con la trasparenza si è potuto mettere a confronto il presente con il passato delle fotografie-chiave, con l'aiuto di brevi racconti audiovisivi che hanno completato l'esplorazione con ulteriori informazioni e brevi storie.

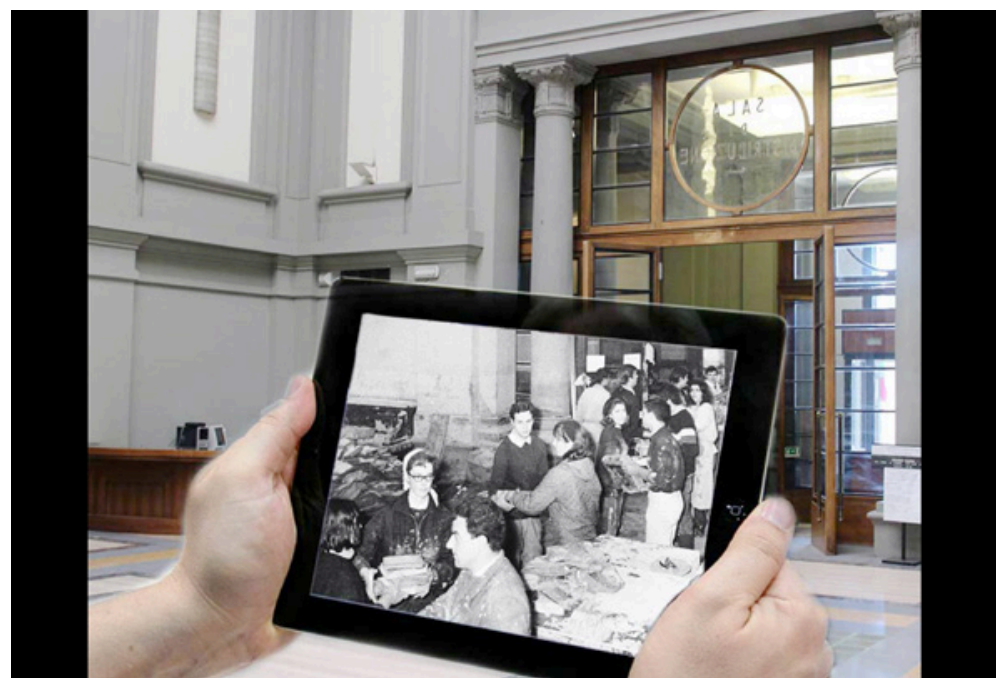

Figura 4 Gli angeli del fango al lavoro. Nanof, elaborazione grafica per concessione degli autori

Le persone che hanno partecipato costituivano un gruppo eterogeneo per età e professione, compreso il personale in pensione della biblioteca; tutti insieme hanno partecipato alle visite guidate silenziose in una sorta di 'safari della memoria': dall'atrio alle sale del pianterreno, dai sotterranei al chiostro del primo piano per circa un'ora in gruppi di massimo 25 persone. 


\section{$5 \quad$ Visite virtuali}

Il progetto Safari della memoria ha previsto inoltre la creazione di tour da fare con dei visori di realtà virtuale accessibili: all'ingresso della biblioteca (ancora fruibili al sito ARNO66ar.it), ${ }^{3}$ attraverso dei percorsi (video-gallery a $360^{\circ}$ ) e con una serie di tappe all'interno della biblioteca dove era possibile attivare i contenuti (immagini e video) relativi ai luoghi attraversati [figg. 5-6].
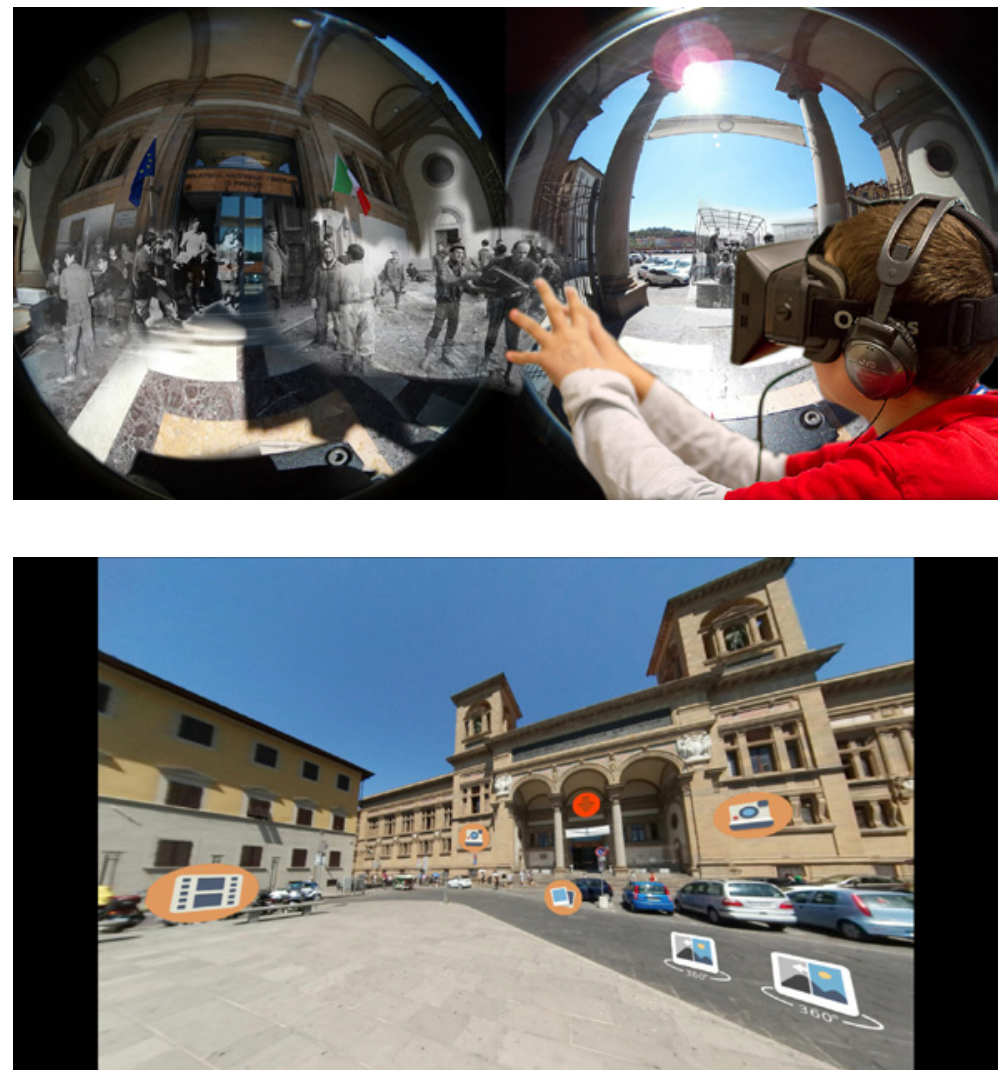

Figure 5-6 I tour virtuali. Nanof, elaborazione grafica per concessione degli autori 


\section{II sito ARNO66ar.it}

La visita virtuale è ancora, in parte, fruibile sul web, ${ }^{4}$ attraverso un sito dedicato all'alluvione di Firenze integrato alla piattaforma internazionale HistoryPin e a Google StreetView / Google Map.

Le immagini sono state geolocalizzate sulla piattaforma HistoryPin, sempre accessibile dal sito ARNO66ar.it, da cui si ha accesso sia alla realtà virtuale ma anche a quella geolocalizzata all'interno della città [fig. 7].

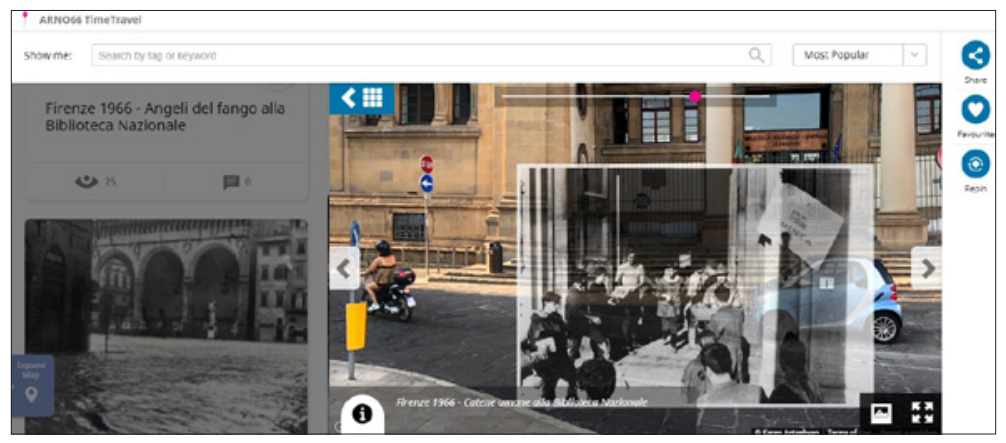

Figura 7 La BNCF su HistoryPin. Nanof, elaborazione grafica per concessione degli autori

\section{$7 \quad$ L'app ARN066 TimeTravel}

La scelta di utilizzare un portale come quello di History Pin non consentiva una grande facilità di accesso passeggiando per la città, per ovviare a questo inconveniente è stata realizzata un'app chiamata ARNO66 TimeTravel, il cui scopo era anche quello di proseguire la visita interattiva iniziata in BNCF nel resto della città. Ancora disponibile su Google Play e sull'Apple Store, l'app bilingue, inglese e italiano, è rivolta principalmente a cittadini, turisti e famiglie o a tour guidati [fig. 8].

Sulla mappa di Firenze sono stati riportati i luoghi di ripresa di alcune delle foto più significative degli eventi del novembre 1966. Le tappe sono organizzate in un percorso che possa raccontare gli eventi principali dell'alluvione, corredate da informazioni multimediali (schede, audio/video). Una volta raggiunti i punti segnalati e, dopo aver trovato la stessa inquadratura dell'immagine fotografica, è possibile giocare con la trasparenza per passare dal presente al passato e viceversa, e accedere a informazioni supplementari relative alle fotografie e ai luoghi colpiti dall'alluvione. 


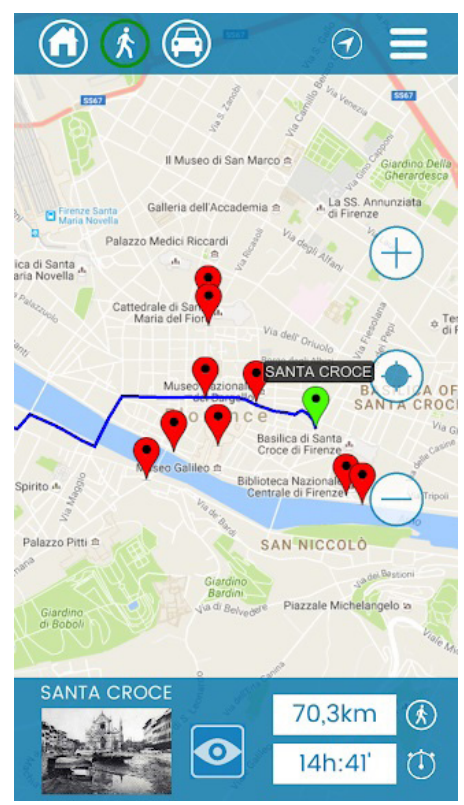

Figura 8 Schermata della app ARNO66 TimeTravel. Nanof, elaborazione grafica per concessione degli autori

\section{Digitalizzazione dell'archivio fotografico}

Nel catalogo online della Biblioteca (OPAC) sono state schedate e digitalizzate ad alta risoluzione circa 3.500 immagini. Una parte cospicua delle immagini è stata organizzata in un database fruibile online in parte sul sito ARNO66ar.it. Per le immagini di cui la biblioteca non possiede il copyright la consultazione è consentita solo in sede.

Un nome che si ripete spesso è quello di Peter Waters, storico responsabile del laboratorio di restauro nella fase di emergenza dopo l'alluvione; sua moglie Sheila Waters ha concesso l'uso alla BNCF di circa 1.100 immagini [fig. 9].

Le immagini furono donate alla biblioteca nel 2008 da Sheila Waters, dopo la morte per asbestosi del marito nel 2003. Quando gli fu diagnosticata la malattia Peter decise di digitalizzare tutte le sue immagini dell'alluvione e ne fece una piccola descrizione che adesso è disponibile sull'OPAC della BNCF. 
Alessandro Sidoti

L'alluvione del 1966 e la Biblioteca Nazionale. Un 'safari' della memoria

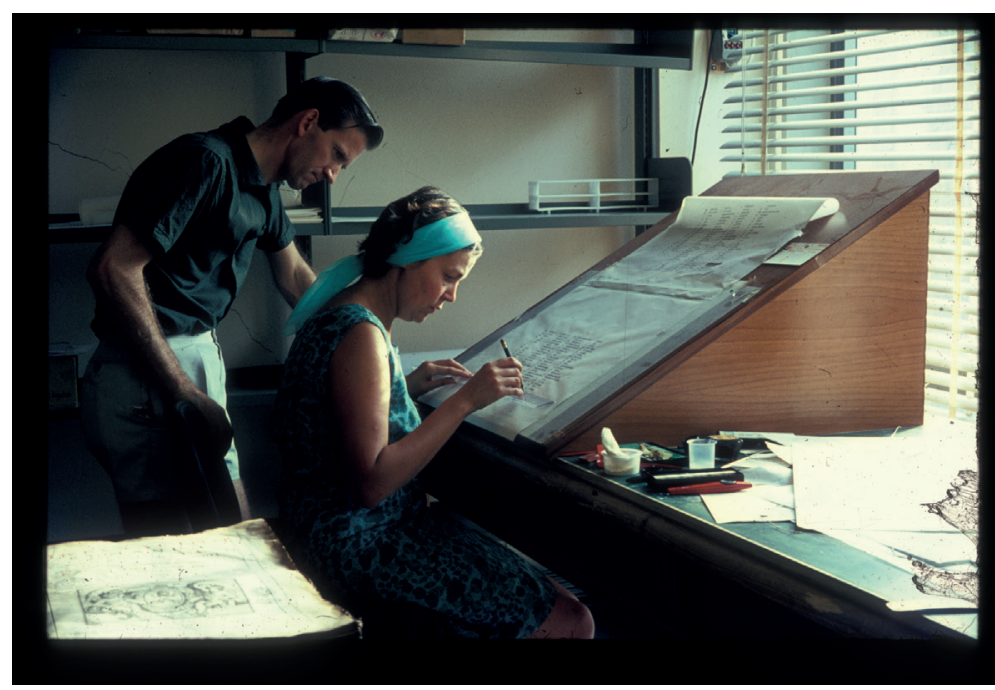

Figura 9 Sheila e Peter Waters al lavoro nel post-alluvione. Peter Waters per concessione degli ered

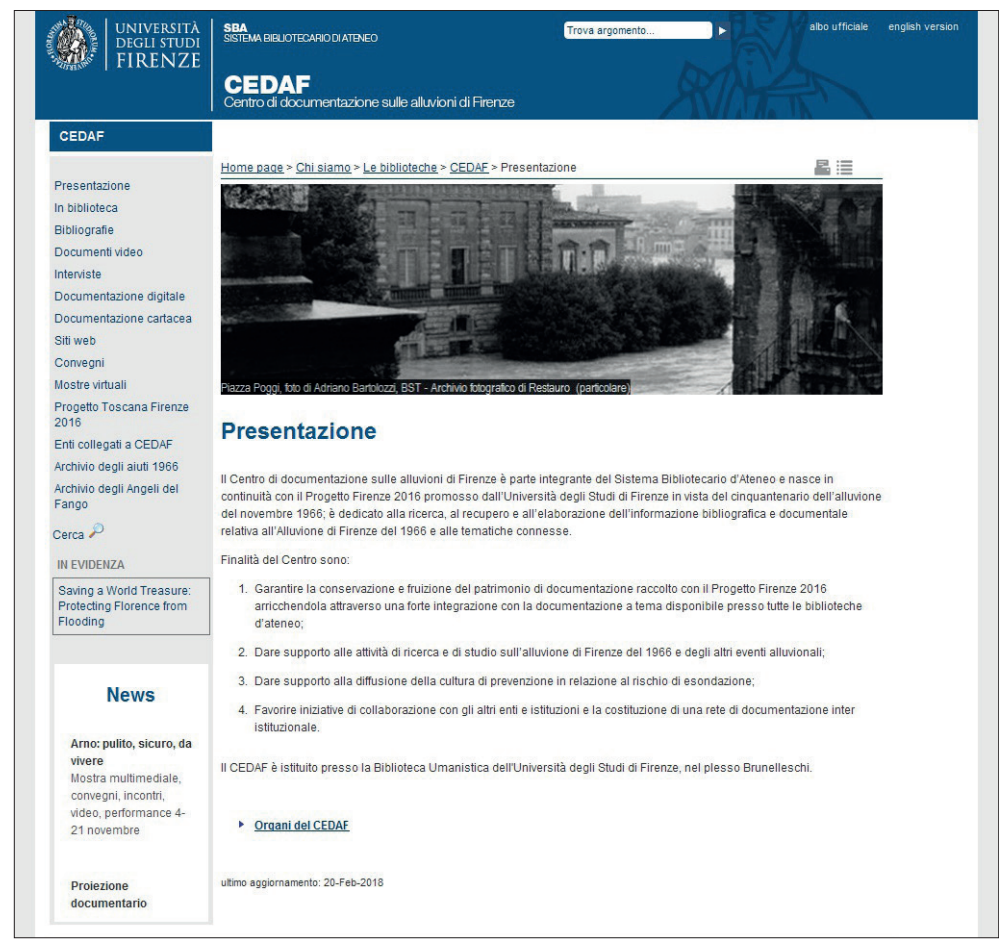




\section{II CEDAF}

Un altro progetto che ha visto la collaborazione di molti altri istituti nello stesso momento è il Centro di documentazione sulle alluvioni fiorentine $(\mathrm{CEDAF})^{5}$ e rappresenta un'altra eredità delle iniziative promosse per il cinquantennale dell'alluvione. In questo caso, le numerose istituzioni colpite dall'alluvione si sono coordinate nel raccogliere tutte le testimonianze in un unico portale [fig. 10]. Il Centro di documentazione garantisce l'accesso alle informazioni delle singole istituzioni che riguardano l'alluvione nel tentativo di conservare una memoria che è a rischio di essere perduta.

\section{Bibliografia}

Acidini Luchinat, C.; Capretti, E. (a cura di) (2016). Firenze, 1966-2016. La bellezza salvata = Catalogo della mostra (Firenze, 1 dicembre 2016-26 marzo 2017). Livorno: Sillabe.

Alessandri, S.; Fontana, A.I. (a cura di) (2006). Contro al cieco fiume: quarant'anni dopo = Catalogo della mostra (Firenze, 4 novembre-16 dicembre 2006). Siena: Protagon Editori.

Batini, G. (1967). 4 novembre 1966 a Firenze: l'Arno in museo. Gallerie, monumenti, chiese, biblioteche, archivi e capolavori danneggiati dall'alluvione. Firenze: Bonechi.

Guasti, G. (2020). ... volta la carta: come cambia il restauro dopo l'alluvione. http://storia.bncf.firenze.sbn.it/passato-2/lalluvione/volta-la-carta-come-cambia-il-restauro-dopo-lalluvione/.

Maini, R. (1996). «Un'occasione perduta. I problemi aperti dall'alluvione nella testimonianza di Luigi Crocetti». Biblioteche oggi, 14(10), 20-3.

5 https://www.sba.unifi.it/p160๑.html. 



\title{
La cultura in fuga San Lorenzo Escape e la storia raccontata per enigmi
}

\section{Daniele Ferraiuolo}

Università degli Studi Suor Orsola Benincasa; Associazione San Bonaventura Onlus, Napoli, Italia

\begin{abstract}
The San Bonaventura Onlus Association was founded in 2014 within the Secular Franciscan Order (OFS), setting itself the goal of offering its contribution in the field of the enhancement of Cultural Heritage. In 2018, it created the first escape room dedicated to libraries and archives, inside the Fra Landolfo Caracciolo library in the Complex of San Lorenzo Maggiore in Naples. Starting from experiences conducted by the Association in the field of digital storytelling, gamification and development of digital tools for the enhancement of ancient books and documents, this contribution illustrates the particularities of the San Lorenzo Escape $\odot$, as well as the methods and skills used for its realization.
\end{abstract}

Keywords Escape room. Multimedia installations. Archives and libraries. San Lorenzo Maggiore, Naples. Enhancement of cultural heritage.

Sommario 1 Premessa. - 2 San Bonaventura Onlus nel panorama dell'innovazione. 3 Le nuove frontiere della narrazione e il progetto San Lorenzo Escape@.

\section{Premessa}

Oggi i prodotti dell'innovazione si misurano, si valutano e, non di rado, si criticano. Potremmo chiederci in quale misura la digital transformation favorisca le azioni di valorizzazione dei beni culturali e in che modo l'educazione al patrimonio culturale tragga beneficio dall'utilizzo di strumenti 'alternativi', utili ad attrarre il pubblico in vario modo. 
Prima di affrontare questo argomento, è utile ricordare che l'art. 27 della Convenzione sulla conservazione del patrimonio mondiale culturale e naturale dell'UNESCO raccomanda agli Stati Membri di fare il «tentativo nel modo più appropriato, e nello specifico con programmi educativi e di informazione, di consolidare nei propri cittadini il riconoscimento ed il rispetto del patrimonio culturale e naturale». ${ }^{1}$ Tale assunto è ribadito e rafforzato, con attenzione alle specificità del patrimonio italiano, nel III Piano nazionale per l'educazione al patrimonio culturale (2018-19), dove si focalizza l'attenzione sulla necessità di favorire non solo l'accesso ma anche la partecipazione di tutti i cittadini allo sviluppo e alla conoscenza del patrimonio. Sui presupposti del rapporto Skills, Training and Knowledge Transfer in Cultural Heritage curato da Voices of culture, ${ }^{2}$ il Piano ritiene strategico e imprescindibile un approccio in grado di integrare le competenze caratteristiche delle 'professioni culturali tradizionali' con le capacità messe in campo dai professionisti impegnati nella 'mediazione del patrimonio', finalizzate alla comunicazione e dunque alla comprensione del patrimonio da parte di tutta la popolazione. ${ }^{3}$

Da entrambi i testi emerge quindi la volontà di intervenire sui modelli tradizionali e di aggiornare, o meglio trasformare, gli strumenti in uso nei luoghi della cultura. Tuttavia, benché la tendenza degli ultimi anni sia stata quella di agevolare l'utilizzo delle nuove tecnologie digitali così da incoraggiare la frequentazione di questi luoghi e il coinvolgimento 'sensoriale' dei visitatori, un recente rapporto dell'Osservatorio Innovazione Digitale dei Beni e Attività Culturali (maggio 2019) denota una certa lentezza nell'adottare tali cambiamenti. L'Osservatorio rileva che dei luoghi della cultura italiani - inclusi quelli che afferiscono a piccole e grandi aree archeologiche o complessi monumentali - il 16\% ha adottato la realtà virtuale per il supporto alla visita in loco, il $12 \%$ la realtà aumentata e solo il $10 \%$ ha scelto il metodo ludico con particolare riferimento all'ambito del gaming. Pur tenendo conto di una lieve crescita percentuale rispetto all'anno precedente, questi dati lasciano pertanto trasparire il prevalere delle intenzioni sulle effettive applicazioni, come dimostra altresì il fatto che il 17\% delle istituzioni culturali abbia dichiarato di avere un'applicazione mobile (app) mentre il 62\% preveda invece di realizzarla. ${ }^{4}$

1 http://whc.unesco.org/en/conventiontext.

2 https://voicesofculture.eu/skills-training-knowledge-transfer-in-cultural-heritage/.

3 https://dger.beniculturali.it/wp-content/uploads/2019/05/PNE_allegato.pdf.

4 Questo rapporto evidenzia anche altri dati rappresentativi delle attività di prenotazione e acquisto dei biglietti di ingresso o di partecipazione a eventi ed esperienze di vario genere. È dimostrato che i canali online sono meno utilizzati rispetto a quelli fisici. Nonostante il 78\% dei Musei dichiari di avere un sistema di biglietteria, solo 
Al di là di questi numeri, è da valutare attentamente il fatto che nell'ambito dei luoghi della cultura i musei continuino a rappresentare, almeno per il momento, gli attori maggiormente interessati all'utilizzo delle nuove tecnologie. In un panorama generale di per sé in crescita, il mondo delle biblioteche e degli archivi fa invece ancora fatica ad adeguarsi. In realtà non si è del tutto consapevoli dei reali benefici derivanti dall'innovazione digitale, in termini di potenziale didattico, educativo o addirittura turistico. Poiché questi luoghi condividono con il territorio non solo la storia ma anche le storie, l'uso degli strumenti digitali può influire molto positivamente sull'efficacia delle attività di comunicazione e narrazione al pubblico. Essi presentano dunque un valore intrinseco che va al di là della loro bellezza o antichità e che ne fa dei posti da visitare, oltre che da frequentare a fini di studio e ricerca.

\section{San Bonaventura Onlus nel panorama dell'innovazione}

Lasciando da parte la grande sfida alla quale si sta assistendo sia in ambito librario sia in quello archivistico italiano in termini di accessibilità alle risorse digitali, ${ }^{5}$ il focus del presente contributo vuole essere quello di cogliere il rapporto tra spazio, contesto, contenuto e visitatore facendo riferimento ad alcune esperienze condotte negli ultimi anni dall'Associazione San Bonaventura Onlus, la cui sede ha ospitato il Convegno di cui qui si riportano gli Atti. ${ }^{6}$

L'Associazione nasce nel 2014 nell'ambito dell'Ordine Francescano Secolare (OFS) ponendosi da subito l'obiettivo di offrire il proprio contributo in materia di valorizzazione dei beni culturali, con una parti-

il 20\% consente l'acquisto online e solo l' $8 \%$ dà la possibilità di effettuare l'ingresso senza stampare il biglietto su carta. Per una panoramica sul Rapporto vedi https:// www.osservatori.net/it_it/osservatori/comunicati-stampa. Gli Atti dei Convegni dell'Osservatorio sono in formato digitale (vedi Bibliografia).

5 Si pensi ai grandi progetti di digitalizzazione promossi e finanziati, in tutto o in parte, dalla Fondazione Polonsky. Tra questi, il DigiVatLib @ della Biblioteca Apostolica Vaticana e il recentissimo lavoro di digitalizzazione degli incunaboli delle biblioteche monastiche in Italia, della Biblioteca Nazionale Centrale di Roma. È utile, a tal proposito, menzionare un terzo progetto finanziato dalla stessa Fondazione, che si è posto l'obiettivo di proporre un approccio globale al manoscritto che non limita le operazioni alla sola digitalizzazione ma anche alle altre attività che ruotano attorno allo studio e alla valorizzazione del patrimonio librario. Si tratta del programma Francia-Inghilterra, 700-1200: manoscritti medievali della Bibliothèque nationale de France e della British Library, per il quale vedi Denoël 2019; Siri 2019. Per ciò che concerne invece i documenti, un progetto di grande interesse e di indiscutibile utilità è rappresentato da monasterium.net $\left.{ }^{(}\right)$, coordinato per l’Italia da Antonella Ambrosio. Vedi Ambrosio 2010, 2019.

6 L'Associazione San Bonaventura Onlus ha sede nella Biblioteca Fra Landolfo Caracciolo del Complesso Monumentale di San Lorenzo Maggiore in Napoli, gestito dai Frati Minori Conventuali di Napoli. 
colare sensibilità verso le biblioteche e gli archivi ecclesiastici e degli ordini conventuali. A partire dal 2016, oltre alla convenzione con l'Ordine dei Frati Minori Conventuali ormai divenuto un elemento imprescindibile, questo sodalizio ha avviato attività di collaborazione per ricerca di fonti di finanziamento e realizzazione di progetti con venti istituzioni tra biblioteche e archivi, tra cui la Biblioteca e Archivio Storico della Diocesi di Alife-Caiazzo (CE), ${ }^{7}$ con la quale sono state portate avanti molte delle iniziative di carattere scientifico e divulgativo.

Archivi e biblioteche offrono la possibilità di essere 'sviscerati' poiché dallo scritto e dalla materia può essere estrapolata, e dunque raccontata, la nostra memoria storica. Per raggiungere le nuove generazioni, e non solo, il mondo dei libri e dei documenti necessita di strumenti efficaci, di facile e immediata consultazione. L'Associazione ha dovuto tenere conto di questo cercando di calibrare le proprie attività sulla base di un concetto fondamentale, quello di edutainment. ${ }^{8}$ Tale strategia, basata sulla fusione tra il fine educativo e quello del divertimento, induce il fruitore, informandolo, a sviluppare le capacità operative tramite attività che coinvolgono diverse forme di intrattenimento.

Oltre alle mostre culturali di tipo interattivo, anche la proiezione di filmati 3D e 5D, l'e-Learning, i software e le installazioni multimediali possono rappresentare strumenti in grado di coniugare intrattenimento e apprendimento. Questo orientamento è entrato a far parte del modo di concepire, da un lato, i servizi per i visitatori e di comunicare, dall'altro, anche con uno dei più complicati target di visitatori di archivi e biblioteche, quello dei bambini e dei ragazzi.

Il mondo dei codici, dei libri antichi e dei documenti d'archivio appare ancora ai più giovani come un ambito esplorativo poco affascinante o avvincente e per tale motivo ha bisogno di metodi e strategie che stimolino l'interattività e l'interpretazione. Tale approccio è senza dubbio affascinante ma proprio per questo necessita di essere portato avanti con una certa cautela, in quanto contiene in sé il rischio di favorire il divertimento ponendo in secondo piano l'aspetto educativo. Ciò può dunque portare facilmente a situazioni in cui l'audience non considera più l'apprendimento come uno degli obiettivi, ma come una sorta di ostacolo al divertimento (Bodnár 2019, 22; Salomon 1983). ${ }^{9}$

7 La biblioteca e l'archivio storico sono diretti dal dott. Luigi Arrigo.

8 Il termine edutainment, una crasi dei sostantivi education e entertainment, è stato coniato nel 1973 da Robert Heyman durante la preparazione dei documentari per il National Geographic, partendo da esperienze già messe in atto da Marshall McLuhan (McLuhan 1964). Sull'argomento, benché con un'attenzione particolare all'ambito dei musei, vedi Okan 2003; Jegers, Wiberg 2003.

9 Su questo aspetto, legato in particolare ai rischi e ai benefici dell'edutainment, vedi Goodland, McIvor 1998; White, Hayward, Chartier 2004. 
Si è resa necessaria innanzitutto la formazione di un gruppo di lavoro composto da professionisti con competenze quasi mai sovrapposte e per questo molto variegate, in grado di far fronte ad esigenze diversificate. ${ }^{10}$ Partendo da una lunga e proficua stagione di digitalizzazione e catalogazione di antichi volumi a stampa, manoscritti, documenti membranacei e cartacei custoditi in diverse istituzioni del centro e sud Italia, ${ }^{11}$ l'Associazione è giunta ad elaborare strumenti, installazioni e applicazioni con l'obiettivo di facilitare il dialogo con il visitatore, che costituisce ora parte attiva e non più passiva.

Sulla base dei risultati ottenuti in termini di riproduzione, ad altissima definizione, di risorse digitali di vario tipo, si è tentato di offrire in primo luogo esperienze che conducono l'utente alla scoperta dei luoghi, dei tempi e delle storie che si celano dietro le carte, sfruttando in sostanza le tecniche del digital storytelling. In secondo luogo, un'attenzione particolare è stata riservata all'uso di tecnologie digitali in grado di dirigere l'interesse sulla materialità e sull'aspetto grafico dei documenti, nonché sulle attività che ruotano attorno alla loro conservazione: dalla catalogazione/descrizione al restauro.

Le installazioni interattive di tipo multimediale realizzate in questi anni sono state concepite non solo per essere ospitate in biblioteche e archivi, e rendere la visita in loco più coinvolgente, ma anche per raggiungere in maniera mirata il pubblico in occasione di particolari eventi culturali: come saloni del libro e mostre librarie e documentarie..$^{12}$ Per citare degli esempi, lo Scaffale 3D interattivo ${ }^{13}$ consente, come farebbe una macchina del tempo, di proiettare il visitatore in luoghi accessibili ma allo stesso tempo distanti o addirittu-

$10 \mathrm{Al}$ momento, il gruppo è rappresentato da archivisti, bibliotecari, archeologi, paleografi, catalogatori, web e industrial designers, sviluppatori di applicazioni e installazioni multimediali.

11 Tra i diversi progetti, è opportuno menzionare quello del 2015 di digitalizzazione dei fondi librari antichi delle biblioteche francescane di Afragola (NA) e Piedimonte Matese (CE) e della Biblioteca e Archivio Storico della Diocesi di Alife-Caiazzo (CE), nell'ambito dell'Obiettivo Operativo 1.10 del POR FESR 2007-2013. Tra il 2016 e il 2017 l'Associazione ha gestito, per la Pontificia Facoltà Teologica dell'Italia Meridionale, un progetto di digitalizzazione di manoscritti cinesi e testi di particolare valore.

12 Si pensi, ad esempio, al Salone del libro e dell'editoria ospitato presso la sala del Refettorio del Complesso Monumentale di San Domenico Maggiore di Napoli, nei giorni 24-27 maggio 2018. L'Associazione è stata coinvolta come partner, inoltre, nell'ambito della mostra documentaria interattiva Hoc instrumentum scripsi ego. Il notariato caiatino nelle pergamene dell'Archivio dell'antica Diocesi di Caiazzo, curata da chi scrive e tenutasi all'Archivio vescovile di Caiazzo (CE) dal 25 marzo al 2 aprile 2017 (Ferraiuolo 2017). In considerazione della grande affluenza e dell'efficacia in termini didattici, la mostra è stata prorogata fino al 2019.

13 L'app è stata realizzata nel 2015 per San Bonaventura Onlus da Mario Tartaglia: (http://www.e-matese.com). I contenuti (selezione e digitalizzazione degli oggetti) sono stati realizzati dalla Cooperativa Effatà. L'interfaccia grafica e le ricostruzioni 3D sono state curate da Melania Zappa. Hanno collaborato, in qualità di esperti in materia di digitalizzazione, catalogazione e analisi del materiale librario antico, Luigi Arrigo e Fabio Brandi. 
ra del tutto scomparsi. L'ambientazione 3D è quella dell'antica biblioteca francescana di Sant'Angelo in Palco di Nola (NA) al cui interno scaffali, libri, tavoli e leggii sono stati realizzati in modo fedele agli originali così da conferire all'ambiente un aspetto suggestivo che garantisce al fruitore un'esperienza immersiva [fig. 1].

Il sistema hardware è composto da un potente PC fisso con scheda video dedicata di ultima generazione, un proiettore ad altissima risoluzione e un sensore Kinect che consente il tracking delle mani senza la necessità di adoperare un controller per l'interazione con l'ambiente. Attraverso il sensore il visitatore può dunque interagire con lo spazio con estrema precisione e naturalezza, avendo la possibilità di toccare virtualmente gli oggetti, spostarli, prendere un libro - tra quelli digitalizzati ad hoc e facenti parte dei fondi della biblioteca ospitante - dallo scaffale, sfogliarlo e analizzarne i dettagli attraverso la 'lente di ingrandimento'.

Questo tipo di approccio consente, come si diceva, anche di entrare a contatto con le pratiche di catalogazione, analisi o restauro condotte prevalentemente in ambito librario. L'installazione multimediale Laboratorio di restauro ${ }^{14}$ ha lo scopo di illustrare all'utente le diverse fasi del restauro conservativo. Progettata per essere utilizzata su schermi touch di grandi dimensioni (L.I.M. o tavoli interattivi), l'installazione consente di interagire in modo intuitivo con l'oggetto da restaurare. Attraverso l'uso dei polpastrelli sulla superficie touch, il visitatore può simulare le operazioni di pulitura e spolveratura del volume, il lavaggio, la sutura degli strappi e l'integrazione delle parti perdute o danneggiate.

Gli strumenti interattivi consentono di entrare a contatto persino con le regole di rilevamento dell'impronta tipografica che, come sappiamo, consistono nella creazione di una stringa composta da lettere e numeri ricavati in punti specifici del libro antico, in grado di identificarne l'edizione e dunque l'esemplare. Un sistema che vale la pena ricordare è quello messo a punto tra il 2015 e il 2016 con l'obiettivo di guidare l'utente nell'identificazione della sequenza alfanumerica all'interno di un ambiente di gioco progettato per segnalare le parti errate e quelle esatte dei fogli [fig. 2]. ${ }^{15}$

14 L'installazione multimediale interattiva è stata realizzata nel 2015 per San Bonaventura Onlus da Mario Tartaglia. I contenuti (selezione e digitalizzazione degli oggetti) sono stati realizzati dalla Cooperativa Effatà. L'interfaccia grafica e le ricostruzioni 3D sono state curate da Melania Zappa. Hanno collaborato, in qualità di esperti in materia di trattamento del materiale librario e archivistico, Luigi Arrigo e Fabio Brandi.

15 L'app è stata realizzata nel 2015-16 per San Bonaventura Onlus da Mario Tartaglia. I contenuti (selezione e digitalizzazione degli oggetti) sono stati realizzati dalla Cooperativa Effatà. Hanno collaborato, in qualità di esperti in materia di catalogazione del materiale librario antico, Luigi Arrigo e Fabio Brandi. È utile precisare che per raggiungere l'obiettivo fissato è opportuno che l'utente sia affiancato nell'utilizzo dell'installazione da personale in possesso di specifiche competenze sull'argomento. 
Daniele Ferraiuolo La cultura in fuga. San Lorenzo Escape e la storia raccontata per enigmi

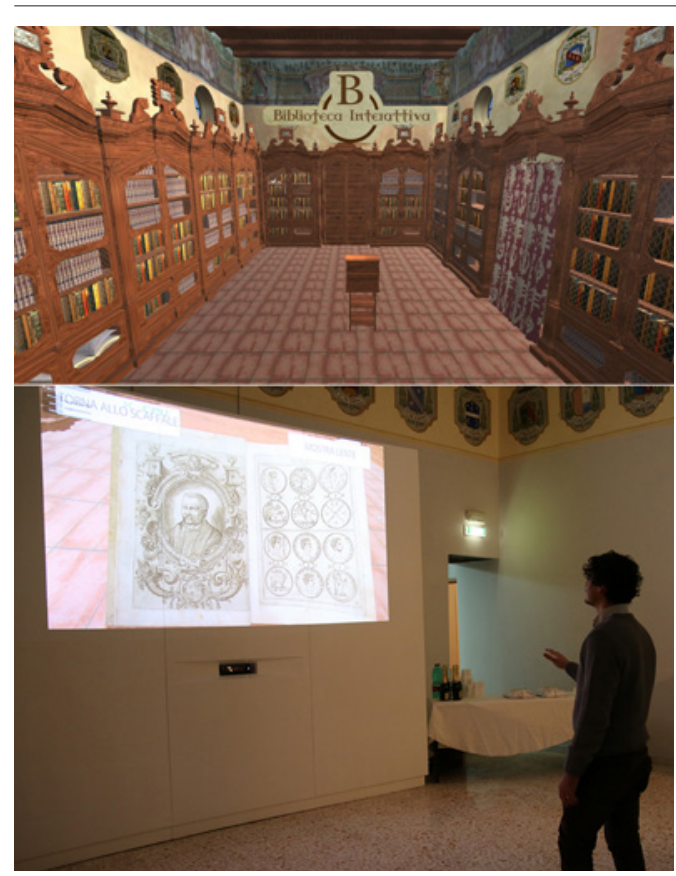

Figura 1 Funzionamento dell'installazione multimediale interattiva Scaffale interattivo 3D $\odot$

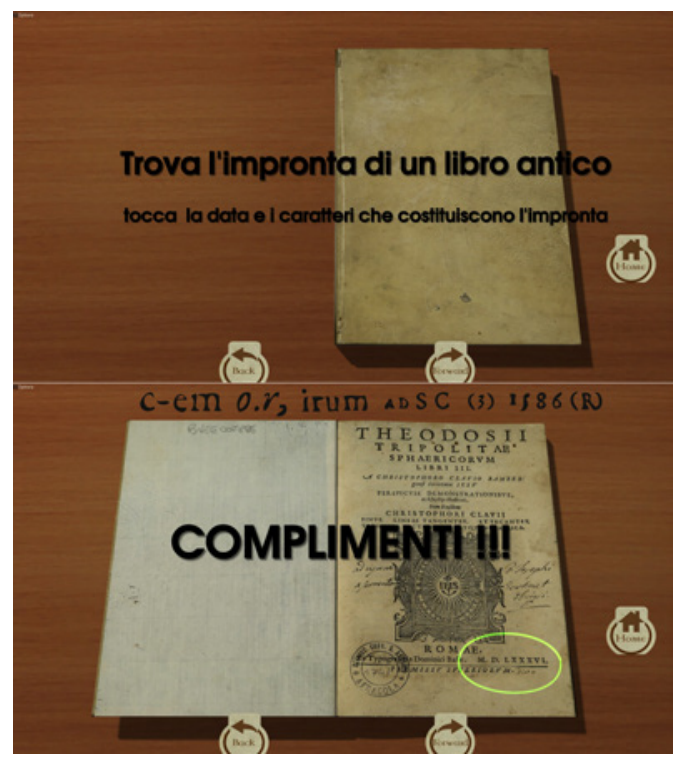

Figura 2 Screenshots dell'installazione multimediale interattiva Calcolo dell'impronta@ (ph. Mario Tartaglia) 


\section{Le nuove frontiere della narrazione e il progetto San Lorenzo Escape $\odot$}

Negli ultimi due anni l'Associazione si è dedicata anche alla ricerca di tecniche di narrazione basate, oltre che sull'uso del racconto supportato da video-installazioni o videoproiezioni, su soluzioni digitali interattive capaci di far convergere gli interessi del pubblico su temi e avvenimenti che ruotano intorno agli oggetti custoditi negli archivi o nelle biblioteche.

Un'indagine sulle lettere dei vescovi al Capitolo di Caiazzo (CE), datate tra il 1474 e il 1749, ha rivelato una serie di dettagli molto interessanti sui rapporti tra i membri della gerarchia ecclesiastica caiatina e i grandi personaggi del mondo dell'arte, soprattutto del periodo rinascimentale. ${ }^{16}$ Grazie a questo lavoro di ricerca ed edizione sono emersi, ad esempio, diversi collegamenti tra il vescovo Giacomo de Lutiis (1480-1506) ed artisti del calibro di Michelangelo Buonarroti e Raffaello Sanzio, con i quali il prelato era entrato in contatto in virtù della sua attività di luogotenente al servizio del cardinale Raffaele Riario (Ferraiuolo 2019, 23-4).

Nell'Archivio Storico Diocesano di Caiazzo, il percorso di apprendimento e di accesso al linguaggio d'archivio è reso ancora più efficace dall'esistenza di un sistema digitale plasmato intorno alla raccolta epistolare ma eventualmente utilizzabile anche per valorizzare altri fondi o raccolte documentarie. Si tratta di un'installazione multimediale sviluppata per illustrare il contenuto dei documenti attraverso la condivisione, su L.I.M., di immagini ad alta risoluzione e di strumenti che ne rendono consultabile il regesto e la trascrizione. Ciò che rende particolarmente affascinante questo strumento agli occhi dei 'non addetti ai lavori' è la funzione che consente, attraverso il passaggio del dito sullo schermo, di evidenziare gli elementi del testo, sciogliere le abbreviazioni e leggere le sigle, ponendo l'utente a stretto contatto non solo con la fonte ma anche con aspetti concernenti il lavoro degli storici, dei paleografi e dei diplomatisti [fig. 3]. ${ }^{17}$

16 La serie Corrispondenza dell'Archivio storico della Diocesi di Alife-Caiazzo consta attualmente di 191 lettere, di cui 101 rappresentano il prodotto delle attività condotte da alcuni prelati nell'esercizio delle proprie funzioni. Si tratta delle sole missive spedite ai canonici che contengono soprattutto disposizioni sul buon andamento della Diocesi e aggiornamenti sulla situazione patrimoniale del Capitolo. Non si conservano le responsive dei corrispondenti. Il corpus è composto in prevalenza da lettere in volgare: di 101 lettere, infatti, solo 9 sono in latino (Ferraiuolo 2019).

17 Nel 2017 l'Associazione ha collaborato, come partner, allo sviluppo dell'app per la Diocesi di Alife-Caiazzo. Dal punto di vista tecnico e informatico, l'applicazione è stata realizzata da Mario Tartaglia. Le attività di digitalizzazione dei documenti sono state condotte da Fabio Brandi (Cooperativa Effatà) e Gianrufo Sparano. Hanno collaborato, in qualità di esperti in materia di analisi del materiale archivistico, esame pale- 


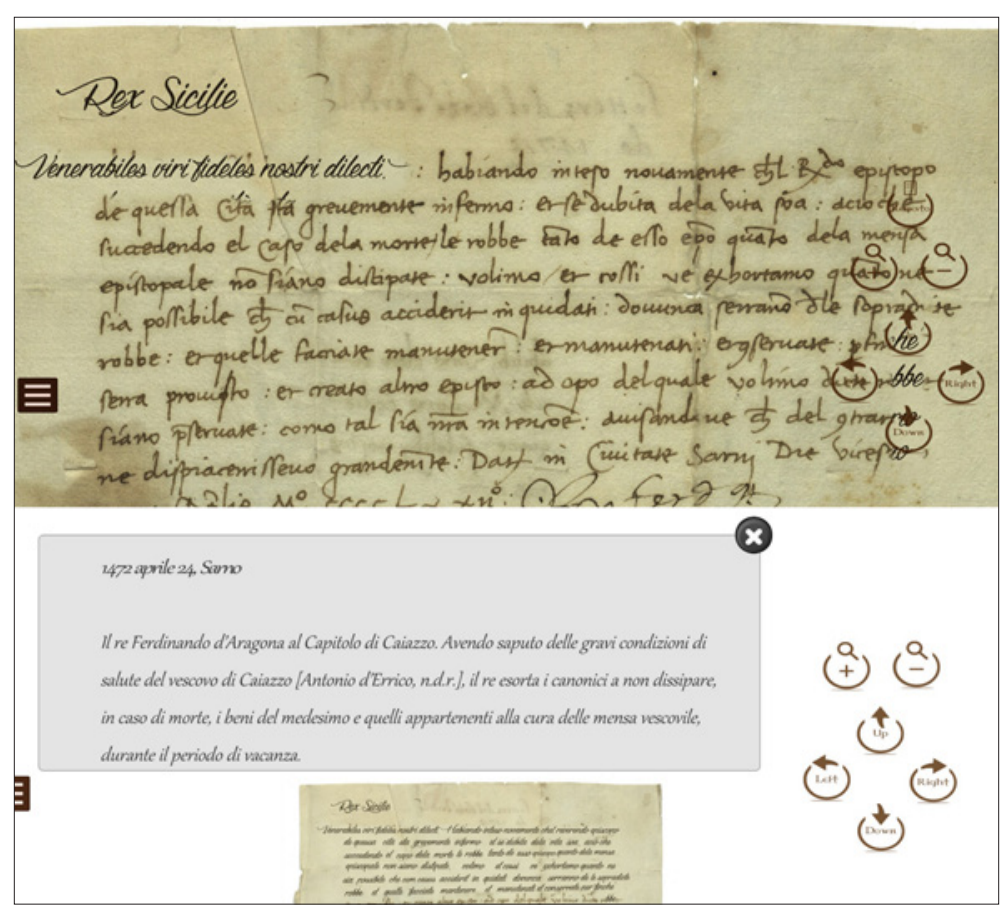

Figura 3 Screenshots dell'installazione multimediale interattiva Lettere dei vescovi@ (ph. Mario Tartaglia)

Il progetto San Lorenzo Escape $\odot$ trae origine dalla commistione di tutte queste esperienze. L'idea è partita dalla volontà di valorizzare ulteriormente il complesso di San Lorenzo Maggiore attraverso la sua biblioteca e il suo archivio, rafforzandone il legame con il territorio e con il patrimonio librario e archivistico posseduto.

Ė opportuno ricordare che il convento di San Lorenzo è fra le realtà più ricche e radicate della Napoli angioino-aragonese e per questo anche fra le più articolate sotto il profilo della documentazione archivistica. ${ }^{18}$ La storia del suo archivio è scandita tuttavia da perdite, dispersioni e dislocazioni, in quanto il convento fu provato da una serie di eventi traumatici a partire almeno dal 1349, anno in cui le sue fabbriche vennero pesantemente danneggiate da un primo terremoto. ${ }^{19} \mathrm{Al}$ di là di eventi abbastanza noti nella storia napoletana

ografico e inventariazione, Luigi Arrigo, Claudia Curcio e chi scrive. L'interfaccia grafica è stata curata da Melania Zappa.

18 Sulla nascita e lo sviluppo del convento vedi Filangieri 1884; Bruzelius 1994; Iannelli 1994; Di Meglio 2003, XXVII-XLI; Autieri 2013.

19 Sulle vicende dell'Archivio di San Lorenzo Maggiore vedi Di Meglio 2003, XLIII-LXII. 
del Cinquecento e del Seicento, ${ }^{20}$ l'Archivio subì un rapido depauperamento a seguito della soppressione del 1808 che portò, nel 1826, al versamento del suo patrimonio nel Grande Archivio del Regno, fra i volumi del fondo Pergamene dei monasteri soppressi. È tuttavia al 1943 che risale il noto incendio di Villa Montesano a San Paolo Belsito (NA) - dove nel frattempo erano stati depositati l'intero Diplomatico del Grande Archivio e i fondi maggiori - che comportò la perdita della documentazione storica di San Lorenzo con la sola eccezione dei fasci cartacei che erano rimasti in Archivio (Di Meglio 2003, XLIV).

Qual è quindi la reale consistenza del posseduto documentario di San Lorenzo, essendo esso in parte perduto e in parte fisicamente decontestualizzato rispetto al suo 'habitat'? L'Archivio di San Lorenzo Maggiore, che al momento convive con la Biblioteca, è ripartito in ulteriori quattro sub-archivi che rispecchiano, in sostanza, le realtà e i personaggi con cui il convento è venuto a contatto nel corso dei secoli.

Gli archivi Brancaccio, Confraternita di Sant'Antonio di Padova, Frati minori conventuali e Palatucci costituiscono una risorsa preziosissima non solo per gli studiosi, ma anche per i visitatori (F. Russo 1991; Battaglia 2003; G. Russo 2017). Un particolare interesse riveste, in questo contesto, il Brancaccio, donato nel 1981 ai Frati minori conventuali dalla principessa Fernanda Ceccarelli. L'importanza di questa raccolta risiede, in primo luogo, nella sua natura mista, membranacea e cartacea, e nel fatto di poter contare circa 250 tra atti di compravendita, privilegi, bolle - compresi tra i primi del Quattrocento e i primissimi dell'Ottocento - e 685 registri cartacei. ${ }^{21}$ In secondo luogo, questo materiale getta luce su aspetti ancora sconosciuti della storia di Napoli, riguardanti nello specifico la chiesa e l'ospedale di Sant'Angelo a Nilo e la Biblioteca Brancacciana, nota per essere la prima biblioteca pubblica della città.

Tornando al progetto San Lorenzo Escape `C, ${ }^{22}$ la sfida è stata, dunque, porsi come obiettivo primario quello di far combaciare le testimonianze a disposizione, e le storie che emergono dalla loro analisi rigorosa, con alcuni degli avvenimenti e dei personaggi che costituiscono parte integrante della trama storica di San Lorenzo. Per raggiungere questo ambizioso obiettivo si è scelto di seguire un filo conduttore alternativo, basato sullo scioglimento di enigmi in un'atmosfera di mistero che fa da sfondo alla prima escape room realizzata nella biblioteca di un convento e incentrata sul tema dei beni librari e archivistici.

20 Come il 9 luglio 1647 quando il convento fu saccheggiato dai rivoltosi guidati da Masaniello in quanto sede dell'armeria della municipalità napoletana (Di Meglio 2003, XLIII).

21 Così ripartiti: Introito ed esito, Duca di Lustri, Incartamenti.

22 Nato da un'idea di Luigi Arrigo e Carlo Caccavale, il progetto è stato ultimato nel 2018 e patrocinato da Effatà, Shadow Computer e Dedalo. Coordinamento scientifico di Laura Del Verme. Teaser al link: http://www.bonaventuraonlus.it/san-lorenzo-escape-napoli/. 


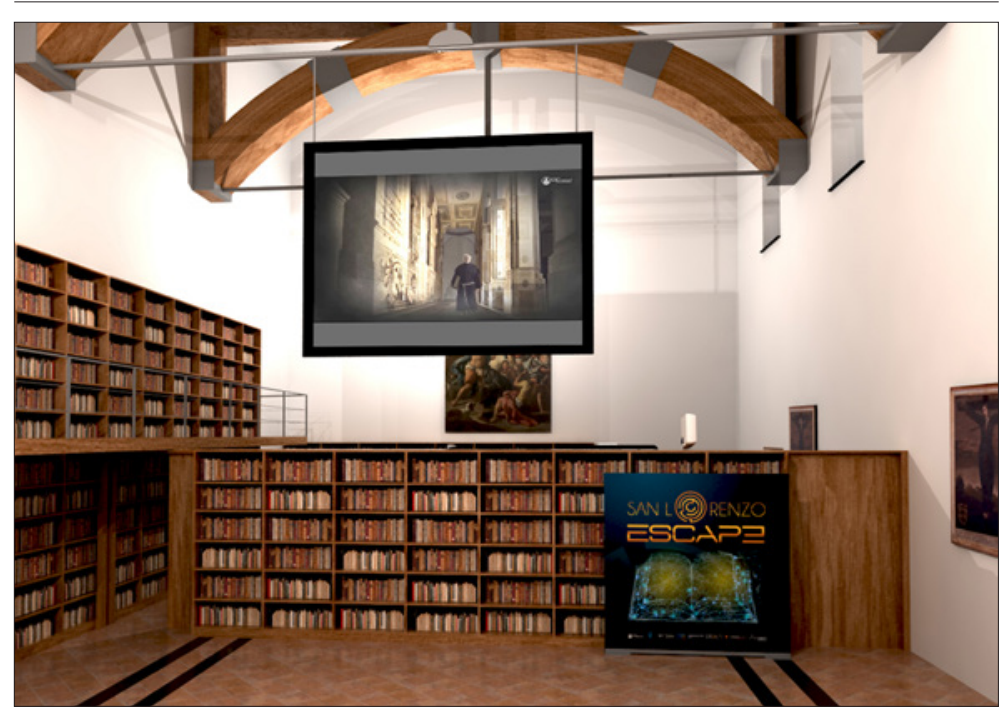

Figura 4 Render dell'area di gioco San Lorenzo Escape@ (ph. Melania Zappa)

La scena, allestita in un'ala della sala di consultazione della Biblioteca Fra Landolfo Caracciolo, ${ }^{23}$ è quella del convento stesso ed è suddivisa in quattro aree tematiche distinte, e tutte accolgono il giocatore nel ripercorrere le orme di alcuni personaggi e avvenimenti [fig.4] . Se il progetto si iscrive in un modello già noto, quello dell'escape room classica, i metodi per lo svolgimento del gioco sono del tutto innovativi in quanto i sistemi di 'sblocco' per il passaggio di stanza, tradizionalmente costituiti da oggetti, lucchetti e serrature, sono rappresentati da codici ottenuti risolvendo i singoli giochi digitali.

Sulla base delle esperienze già maturate e di cui si è dato rapido accenno, ma allo stesso tempo con i dovuti aggiornamenti, la San Lorenzo Escape $@$ propone ai giocatori/visitatori ${ }^{24}$ una serie di enigmi da risolvere attraverso l'utilizzo dei tavoli multimediali e della realtà aumentata. ${ }^{25}$ L'itinerario, scandito in diversi punti dagli interventi virtuali di Landolfo Caracciolo, ${ }^{26}$ si basa sulla storia di indivi-

23 L'allestimento scenografico e il logo sono di Melania Zappa.

24 La San Lorenzo Escape (C) può accogliere fino ad un massimo di 8 persone per sessione di gioco.

25 Le installazioni sono state prodotte e ottimizzate da Mario Tartaglia. Le ricerche storiche, l'ideazione e il testo degli enigmi sono stati curati da chi scrive e da Giovanni Russo.

26 Attraverso videoproiezioni che vedono come protagonista un attore professionista, Peppe Barile. Testi di Laura Del Verme, riprese video, montaggio e Vfx di Marco Flaminio, fotografia di Stanislao Flaminio, traduzioni di Claudia Curcio, Aldo Meola e Margherita Palmieri. 
Daniele Ferraiuolo

La cultura in fuga. San Lorenzo Escape e la storia raccontata per enigmi

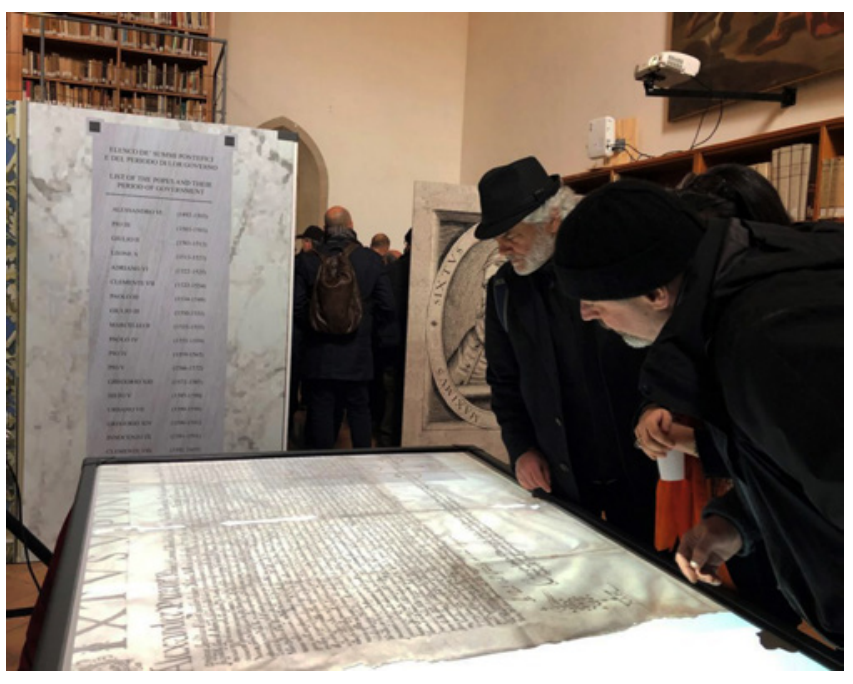

Figura 5 Funzionamento delle installazioni in occasione della presentazione della San Lorenzo Escape $\subset$ del 13 dicembre 2018

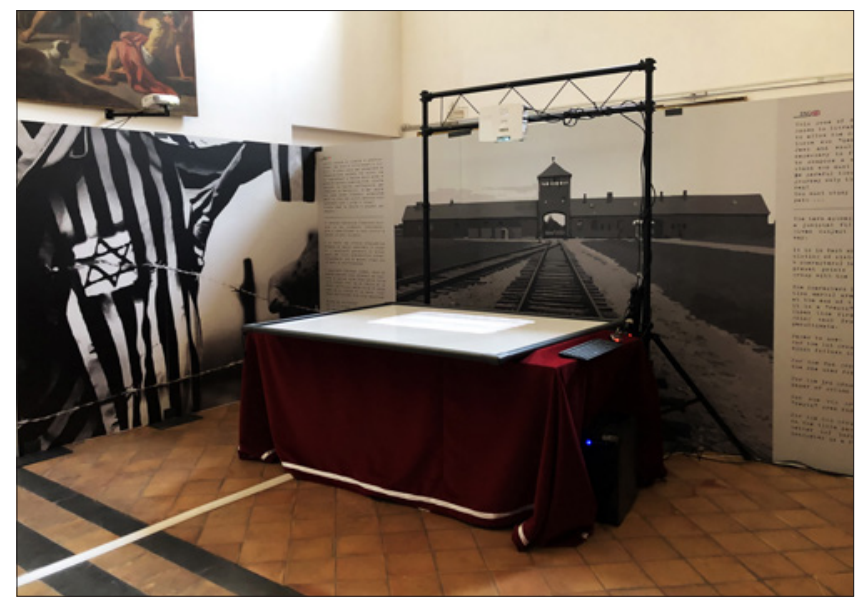

Figura 6 L'ultima area del percorso San Lorenzo Escape(c) dedicata a Giovanni Palatucci (ph. Melania Zappa) 
dui che hanno avuto un impatto fortissimo sulla città di Napoli e sul complesso di San Lorenzo quali, per citarne alcuni, Boccaccio, Masaniello o papa Sisto V.

L'azione della 'fuga', del passare da una stanza all'altra per raggiungere l'uscita, necessita di strategie di gruppo che consentano di superare le diverse prove cimentandosi, sulla base di un'attività ludico-educativa, ${ }^{27}$ nella datazione di un documento e nel calcolo delle calende, nello scioglimento di abbreviazioni, nella consultazione di mappe storiche o nel calcolo dell'impronta [fig. 5]. Intorno a quest'ultima operazione ruota la parte conclusiva dell'itinerario che affronta l'argomento della deportazione nazista attraverso l'analisi delle carte del vescovo Giuseppe Maria Palatucci, e in minima parte di Giovanni Palatucci, conservate nell'Archivio Palatucci [fig. 6]. ${ }^{28}$

Per concludere è utile notare che questo allestimento, ormai divenuto permanente, ha fatto senza dubbio da volano per un netto incremento delle visite. In questo senso, l'attività di promozione e una fitta campagna di comunicazione tramite social, media o canali istituzionali ${ }^{29}$ hanno dato risultati inaspettati, tali da destare un rinnovato interesse nell'utente abituale e curiosità in quello occasionale. La necessità di ampliare il bacino di utenza ha spinto, in aggiunta, a snellire il processo di prenotazione attraverso la sua gestione online. ${ }^{30} \mathrm{Si}$ tratta, in definitiva, solo di un altro tassello di un più ampio progetto di valorizzazione in continuo sviluppo.

27 Questo tipo di approccio non è rivolto solo al target dei bambini o dei ragazzi, ma anche a quello degli adulti.

28 Giuseppe Maria e Giovanni Palatucci sono medaglie d'oro al valor civile della Repubblica Italiana per aver impedito la deportazione di moltissimi ebrei. Giovanni, nelle sue funzioni di questore a Fiume, indirizzò diverse persone verso il campo di internamento di Campagna (SA), dove lo zio era vescovo, tentando di impedirne la deportazione nei campi di sterminio nazisti. È attualmente in corso di pubblicazione una tesi di Dottorato sull'Archivio Palatucci, discussa presso la Pontificia Università Gregoriana da don Michele Di Martino.

29 Tra cui quello scolastico e universitario. In virtù di un Protocollo di Intesa con il Dipartimento di Studi Umanistici dell'Università Federico II di Napoli, l'Associazione è tra i membri del progetto europeo Time Machine Europe (c) e tra i partner del progetto Naples Time Machine $(\subset)$ per attività di valorizzazione e promozione dei beni librari e archivistici, con particolare riferimento all'ambito napoletano.

30 Per la prenotazione l'utente può utilizzare sia il canale Facebook (https://www. facebook.com/sanlorenzoescapenapoli/) sia quello ufficiale dell'app creata da CMH (https://play.google.com/store/apps/details?id=it. cmh. bonaventura\&gl=IT). 


\section{Bibliografia}

Ambrosio, A. (2010). «Il progetto Monasterium in Italia: le prime esperienze a Napoli». Archivi, 5(2), 129-45.

Ambrosio, A. (2019). «Digital Critical Editions of Medieval Documents on Monasterium. Net». Balouzat-Loubet, C. (ed.), Digitizing Medieval Sources. L'édition en ligne de documents d'archives médiévaux. Challenges and Methodologies - Enjeux, méthodologie et défis = Actes du Colloque (Nancy, 9-10 juin 2016). Turnhout: Brepols, 69-84.

Autieri, F. (2013). San Lorenzo Maggiore tra cultura e potere. Il complesso monumentale. Il convento. Napoli; Roma: Edizioni Scientifiche Italiane.

Battaglia, G. (2003). «L'Archivio Brancaccio». San Lorenzo Maggiore. Un patrimonio per la città = Catalogo della mostra documentaria, bibliografica, iconografica (Napoli, 5 novembre-9 dicembre 2003). Napoli: Luciano Editore, 33-5.

Bodnár, D.S. (2019). «Escapism or Active Involvement? A Dimension of Museum Visitor Experience». Budapest Management Review, 11, 18-36.

Bruzelius, C.A. (1994). «ll coro di S. Lorenzo Maggiore e la ricezione dell'arte gotica nella Napoli angioina». Pace, V.; Bagnoli, M. (a cura di), I/ gotico europeo in Italia. Napoli: Electa, 264-77.

Dall'emergenza nuovi paradigmi digitali per la cultura $=$ Atti del Convegno $(27$ maggio 2020). https://bit.ly/30Wul90.

Denoël, C. (2019). «Le programme Polonsky "France-Angleterre, 700-1200: mae nuscrits médiévaux de la Bibliothèque nationale de France et de la British Library": bilan et perspectives». Bulletin du bibliophile, 1, 3-10.

Di Meglio, R. (2003). Il Convento francescano di S. Lorenzo di Napoli. Regesti dei documenti dei secoli XIII-XV. Salerno: Carlone Editore.

Ferraiuolo, D. (a cura di) (2017). Hoc instrumentum scripsi ego. Il notariato caiatino nelle pergamene dell'Archivio dell'antica Diocesi di Caiazzo = Catalogo della mostra (Caiazzo, 25 marzo-2 aprile 2017). Napoli: Edizioni San Bonaventura Onlus.

Ferraiuolo, D. (2019). I vescovi di Caiazzo. Lettere al Capitolo (1474-1749). Napoli: Guida Editori.

Filangieri, G. (1884). Documenti per la storia, le arti e le industrie delle province napoletane, vol. 2. Napoli: Tipografia dell'Accademia Reale delle Scienze.

Goodland, S.; Mclvor, S. (1998). Museum Volunteers: Good Practice in the Management of Volunteers. London: Routledge.

lannelli, P. (1994). Lo studio teologico OFMConv nel San Lorenzo Maggiore di Napoli. Cenni storici e serie dei reggenti, lettori estudenti (1488-1848), guardiani del convento (1482-1990). Roma: Miscellanea Francescana.

Il viaggio nella cultura è digitale: chi guida? = Atti del Convegno (23 maggio 2019). https://bit.ly/2SLzex5.

Jegers, K.; Wiberg, C. (2003). «FunTain: Design Implications for Edutainment Games». Proceedings of ED-MEDIA (Charlottesville, USA, 2003). http:// www8.informatik.umu.se/ colsson/shortjegwib.doc.pdf.

McLuhan, M. (1964). Gli strumenti del comunicare. Milano: Il Saggiatore.

Okan, Z. (2003). «Edutainment is Learning at Risk». British Journal of Educational Technology, 34, 255-64.

Russo, F. (a cura di) (1991). Regesti delle pergamene di S. Angelo a Nilo. La cappella Brancaccio dei Frati Minori Conventuali. Napoli: Archivio Storico Brancaccio. 
Daniele Ferraiuolo La cultura in fuga. San Lorenzo Escape e la storia raccontata per enigmi

Russo, G. (2017). «Gli Archivi». Russo, G. (a cura di), Volumina et digital library in San Lorenzo a Napoli. Sulle orme di san Francesco nella Biblioteca Fra Landolfo Caracciolo = Catalogo della mostra (Napoli, 30 maggio-8 giugno 2017). Napoli: Edizioni San Bonaventura Onlus, 19-23.

Salomon, G. (1983). «The Differential Investment of Mental Effort in Learning from Different Sources». Educational Psychologist, 18(1), 42-50.

Siri, F. (2019). «Digitalizzare i manoscritti medievali? Riflessioni a margine di un progetto internazionale». Digitalia, 14(2), 33-9.

White, R.; Hayward, M.; Chartier, P. (2004). Edutainment: The Next Big Thing = Proceedings of IAAPA Convetion (Orlando, USA, 2004). https://www.whitehutchinson.com/news/downloads/IAAPAEdutainmentSeminar.pdf. 



\title{
Oltre le mostre per promuovere i patrimoni documentari
}

Mariella Guercio

La Sapienza Università di Roma; Associazione Nazionale Archivistica Italiana - ANAI, Italia

\begin{abstract}
This paper discusses the natural difficulties for the archival resources of being handled for exhibition and for massive exploitation not only because institutions and individuals create records for administrative, technical or personal reasons but also because their use implies a specific, complex and time consuming interactive dimension based on a rich and qualified set of descriptive information. Strategic alliances and cooperation as well as specific training initiatives are required for transforming generic and often trivial proposals dedicated to enlarge the archival community and audience into reliable and convincing programs able to share experiences and good practices.
\end{abstract}

Keywords Promotion. Enhancement. Archival exhibitions. Cultural communication. Sharing programs.

Tra i professionisti che operano in campo documentario (certamente in ambito archivistico), l'idiosincrasia per le mostre (soprattutto per quelle che da almeno vent'anni attirano, grazie anche a ricercate soluzioni di marketing, masse di persone di tutte le età e di tutte le provenienze) è atteggiamento diffuso e crescente, che peraltro ha radici consolidate e non solo nel nostro settore. Naturalmente è indispensabile saper distinguere, poiché non tutte le iniziative espositive suscitano la stessa reazione negativa, come del resto sottolineava già nel 2001 un noto critico d'arte e giornalista britannico in un articolo che ancora oggi potremmo considerare di grande attualità, riferendosi al patrimonio storico-artistico e alle già allora invadenti e tutt'altro che qualificate attività di valorizzazione. Scriveva in quegli anni provocatoriamente Jonathan Jones che le mostre (definite blockbuster): 
incoraggiano un approccio idiota nei confronti dell'arte promettendo conoscenze ed esperienze irrepetibili e complete con un atteggiamento che ha sempre un che di pretenzioso e [quindi] di falso. (Giannini 2015)

Le mostre veramente imperdibili (come sostengono del resto rispettati critici d'arte) non mancano, ma sono pochissime: «mostre di ricerca che aggiungono nuovi capitoli nella conoscenza di un artista o di un periodo o di un movimento», oppure mostre "che divulgano nuove scoperte», o ancora «propongono percorsi didattici e divulgativi interessanti e di approfondimento». Tutto fuorché un prodotto di intrattenimento istantaneo, indotto e non reale (Giannini 2015).

Nel caso, poi, delle mostre documentarie, dovremmo aggiungere altri elementi di valutazione tutt'altro che confortanti per quanto riguarda una fruttuosa condivisione delle opere e dei patrimoni esposti, soprattutto se teniamo nel debito conto sia la complessità di lettura e di interpretazione che richiedono perché il visitatore ne comprenda significati, valori, contesti sia l'impegno e le risorse necessari alla loro realizzazione e alla salvaguardia dei beni medesimi oggetto dell'esposizione. Eppure, anche in questo ambito le iniziative non sono mancate negli ultimi decenni, a partire - per il settore degli archivi - da quelle avviate, non a caso, in concomitanza con il passaggio del settore al nuovo Ministero per i beni culturali e ambientali nel 1975 e culminate con la grande, costosa ma presto dimenticata mostra Il tesoro degli archivi nella metà degli anni Novanta. Per quasi un ventennio, dalla nascita del MiBACT, anche il settore archivistico è stato sottoposto a un vero e proprio tour de force caratterizzato da progetti spesso abbinati di esposizioni e convegni. Il citato evento del 1996, Gentium memoria archiva: il tesoro degli archivi, organizzato dall'Ufficio centrale per i beni archivistici grazie ai finanziamenti ottenuti in occasione del semestre italiano di Presidenza dell'Unione europea, richiese un enorme sforzo organizzativo ma fu l'ultimo grande progetto nel settore e, comunque, fu vissuto dai suoi numerosi protagonisti con un buon grado di consapevolezza critica dei suoi limiti. Come, infatti, rilevava la stessa curatrice della mostra, Gabriella Olla Repetto, per l'amministrazione, per i promotori e per i curatori non si trattò di 'un'operazione di valorizzazione' ma di 'un'azione di tutela' finalizzata soprattutto a ricordare che ogni documento archivistico esistente è un 'tesoro' per chi lo ricerca poiché i documenti sono una testimonianza di civiltà e di storia (Olla Repetto 1996). Non a caso, quindi, negli anni successivi e per un lungo tratto, il percorso istituzionale destinato ad assicurare al patrimonio archivistico visibilità e riconoscimento si è concentrato su altre tipologie di eventi significativi, in particolare sulla organizzazione di due conferenze nazionali, la Conferenza nazionale degli archivi tenuta nel luglio 1998 presso l'Archivio centrale del- 
lo Stato, ${ }^{1}$ cui seguì dieci anni dopo una seconda iniziativa altrettanto rilevante. In entrambi i casi gli obiettivi furono quelli di aprire le fonti archivistiche a nuovi pubblici, con un'attenzione particolare per i cittadini e per una utenza in grado di apprezzarne da diversi punti di vista la polifunzionalità e la capacità di raccontare storie.

Il fatto che i documenti non siano nati per essere messi in vetrina è del resto indiscutibile. Nascono per un uso individuale, di studio o di lavoro, per ragioni istituzionali e amministrative e comunque implicano una specifica e ben circoscritta dimensione interattiva: l'esposizione li mette a rischio, è sempre inevitabilmente parziale e, senza laboriosi, costosi e raffinati interventi di mediazione e contestualizzazione, non ne favorisce alcun uso intelligente e produttivo. Lo sottolineava, del resto, già nel 1994 Carlo Federici nel convegno organizzato dalla Fondazione Franceschini I segreti in vetrina. Utilità e danno per la storia delle mostre dei documenti, libri e cimeli (Federici 1996). Già in quella occasione si faceva riferimento alla necessità che la valorizzazione, diffusione, condivisione di questi patrimoni richiedesse un uso impegnativo di strumenti multimediali di navigazione e di collaborazione, alla creazione di spazi e di ambienti virtuali complessi, capaci di dar vita a percorsi immersivi per consentire al visitatore-studioso-lettore di approfondire le conoscenze di cui dispone o di trovare nuovi contenuti e nuovi stimoli di riflessione (Parenti 1995).

Non è quindi per snobismo o pigrizia, ma per convinzione personale di lunga durata che chi scrive non ha partecipato mai all'organizzazione di mostre documentarie, ritenendo che soprattutto nell'ultimo decennio il fenomeno del 'mostrismo' (per riprendere una felice espressione di Armando Petrucci che già nel 1982 stigmatizzava con la consueta efficacia la frenesia espositiva delle istituzioni pubbliche e degli operatori privati) testimonia tutta la sua debolezza e inconsistenza (Petrucci 1982). Tanto più grave se si riflette sulla lunga durissima crisi economica che nel nostro Paese da un lato ha tagliato le risorse per interventi qualificati che avrebbero forse consentito lo sviluppo di iniziative culturali di successo, dall'altro ha concentrato in poche mani esperte, ma non necessariamente sapienti, quelle limitate iniziative di marketing che hanno coinvolto il patrimonio culturale, soprattutto nel settore archivistico e librario.

Non per questo, tuttavia, è consentito sospendere la riflessione e gli interrogativi sulle opportunità concrete e rilevanti che i professionisti del settore e le istituzioni di conservazione delle memorie documentarie devono essere in grado di cogliere per la valorizzazione dei patrimoni e sulle strategie e alleanze da promuovere per individuare percorsi alternativi e credibili di condivisione di iniziative significative. 
Esperienze esemplari di successo e qualità non sono mancate in questi anni, ma è indubbio che nei nostri domini non siamo stati in grado di trasformarle in strumenti consolidati, in capacità di progettazione diffusa, in occasioni - utilizzando le parole di Armando Petrucci - per «programmare e realizzare una politica culturale di lunga durata, tesa ad affrontare e risolvere i nodi reali della conservazione, della conoscenza, dell'uso del nostro patrimonio» (Petrucci 1982, 1159). In poche parole, non abbiamo saputo finora trasformare una rete professionale di operatori e di progetti in programmi di lavoro efficaci e continuativi destinati ad allargare realmente il nostro 'pubblico'.

Eppure il settore ne ha estremamente bisogno, come ci ricorda Claudia Salmini in uno dei pochi interventi didattici dedicato alla comunicazione del patrimonio archivistico nel volume collettaneo $\mathrm{Ar}$ chivistica, sottolineando che:

poche persone in Italia conoscono gli archivi...si rileva un diffuso analfabetismo documentario, riscontrabile in numerosi uffici della pubblica amministrazione, [anche se tutti gli addetti ai lavori sono consapevoli della necessità non rinviabile di] contrastare questa condizione che potremmo definire di 'rimozione collettiva' degli archivi. (Salmini 2014, 337)

Ė difficile rispondere/reagire positivamente a questi nodi senza comprenderne le specifiche ragioni e senza contestualizzare storicamente un fenomeno che potremmo definire di rimozione collettiva o di amnesia generalizzata circa l'esistenza stessa e soprattutto la ricchezza di queste fonti documentarie. Nei secoli passati - scrive sempre Claudia Salmini $(2014,338)$ nel contributo ora citato - una simile ignoranza degli archivi, della loro funzione e del loro valore sarebbe stata impossibile, mentre oggi non possiamo non riconoscere quanto sia diffusa tra gli intellettuali, gli apparati amministrativi, il ceto medio (e talvolta anche tra gli studiosi e i ricercatori). È un fenomeno recente, che risale agli ultimi Cinquanta-Settanta anni, la cui analisi richiederebbe una riflessione approfondita con l'obiettivo di fornire strumenti interpretativi e suggerire proposte di intervento adeguate soprattutto nello specializzare il lavoro di mediazione nei nuovi contesti della comunicazione digitale. Qualche buono studio (Feliciati, Alfier 2013, 2017) è stato condotto in questi anni sul rapporto complesso che il settore archivistico ha con la propria utenza e sui ritardi accumulati soprattutto nell'adattare il tradizionale e difficile lavoro di descrizione delle fonti conservate alla comunicazione in rete. Ė, tuttavia, mancata l'occasione per affrontare con sistematicità nodi pur così vitali per il settore. Anche le analisi più serie non hanno finora saputo offrire indicazioni metodologiche capaci di indirizzare con efficacia e coniugare l'esigenza della condivisione con la complessità di fonti che senza un'accurata e impegnativa descri- 
zione/traduzione disorientano gli utenti meno esperti, proprio quelli che si vorrebbe raggiungere e coltivare.

È del resto nota la difficoltà di trovare risorse per settori di nicchia, soprattutto quello archivistico che ha enormemente sofferto in questi anni per la mancanza di mezzi e di personale, per l'assenza di ricambio e di interventi formativi di aggiornamento. Un processo di impoverimento devastante che ha segnato gravemente le amministrazioni più deboli, da un lato il Ministero dei beni culturali e, al suo interno, gli archivi e le biblioteche, dall'altro gli enti locali la cui azione è stata nell'ultimo decennio ancora più insufficiente nella gestione dei loro patrimoni documentari, anche in conseguenza della perdita di efficienza della tutela e del coordinamento statale e del disinteresse progressivo manifestato dall'intero sistema regionale, ${ }^{2}$ se si escludono le iniziative sviluppate da alcune regioni per la salvaguardia degli archivi digitali.

Non bisogna, infine, tralasciare il tema della formazione e le debolezze delle iniziative di aggiornamento professionale, non tanto per quanto riguarda i contenuti tecnici più specifici, quanto per la limitatezza delle iniziative finalizzate a insegnare ai professionisti a lavorare in team, a progettare e comunicare, a trasformare in sistema, gestire con efficienza e mettere in rete le migliori esperienze e pratiche. Su questi aspetti gli enti formativi hanno manifestato ritardi notevoli. Non si tratta tanto dei corsi di laurea magistrale di archivistica e biblioteconomia, peraltro costretti da vincoli amministrativi così rigidi da renderne faticosa la stessa sopravvivenza. Anche le scuole di specializzazione e i percorsi di alta formazione (dalla Scuola di specializzazione della Sapienza, ai master biennali di II livello di alcuni atenei, alle scuole di alta formazione degli Archivi di Stato) hanno dedicato scarsa attenzione allo sviluppo delle competenze ora ricordate. Lo stesso vale, almeno per l'ambito archivistico, per le attività di aggiornamento condotte dalle associazioni di settore, che si sono finora limitate a qualche tentativo frammentario, destinato inevitabilmente a esiti non entusiasmanti.

Insomma, superare il 'mostrismo' senza rinunciare a una comunicazione efficace si è rivelato in questi anni un compito molto più impegnativo del previsto: un nodo che tutti a parole ritengono cruciale affrontare, ma che per ora si è tradotto, nel migliore dei casi, in un numero ancora molto limitato di proposte promettenti e durature, come quelle che hanno trovato spazio anche nel convegno di Napoli. Poiché le tecnologie sono mature e la consapevolezza cresce, è forse

2 Come dimenticare la prontezza con cui quasi tutte le Regioni hanno accettato, senza protestare, il ritorno allo Stato della tutela del patrimonio bibliografico non statale a seguito della riforma Franceschini del 2015 (art. 16, comma 1sexies del d.l. 78/2015 convertito con modifiche in legge 125/2015). 
arrivato il momento di investire meglio sulle buone pratiche che pur si sono realizzate in questi anni. Per passare dai progetti a un serio e coordinato programma di lavoro, il ruolo delle associazioni professionali è senza dubbio indispensabile per sviluppare forme di collaborazione strategiche inter-associative e inter-istituzionali. Fermo restando che non si tratta di imparare e prendere esempio solo dalle esperienze di successo, poiché spesso gli insuccessi si rivelano anche più istruttivi e proficui. Aiutano per esempio, e non è poco di questi tempi, a non disperdere le risorse, commisurando meglio le energie e concentrando gli sforzi nella direzione finora più trascurata, eppure più convincente se si opera in una prospettiva di lungo periodo: quella di promuovere percorsi di comunicazione che rendano i patrimoni documentari più vivibili ('facendoli respirare', ha scritto qualche commentatore), senza tradirli, senza ridurne o nascondere la complessità e valorizzando non solo i contenuti ma anche le propensioni delle persone (utenti e professionisti) a utilizzarli in forme nuove. In questo processo non possiamo certo ignorare che far crescere nuovi pubblici per gli archivi è difficile e che non è sempre vincente e convincente ricorrere ai grandi player di mercato per ottenere risultati persistenti, così come non basta un bravo esperto di comunicazione per accompagnare con sapienza e con successo i nostri professionisti lungo una strada di sviluppo di competenze qualificate e di iniziative significative. Oltre che competente, il nostro esperto deve infatti sapersi misurare concretamente con le peculiarità dei nostri beni. Infine, è necessario essere consapevoli che il ricorso alla digitalizzazione, pur indispensabile in questo ambito, presenta tante opportunità quanti problemi finora ignorati, tra cui quello impegnativo della conservazione stessa dei contenuti digitali prodotti dagli interventi di valorizzazione. Non è neppure scontata la capacità dei nuovi progetti di assicurare un rapporto ricco tra patrimoni, territorio e identità delle comunità di riferimento. Mantenere alto il livello della consapevolezza critica è quindi un esercizio necessario, così come dobbiamo mettere in campo, con la serietà cui i nostri mestieri ci hanno abituato, un'etica della collaborazione che riconosca da un lato che non siamo 'comunicatori' né dobbiamo diventarlo, dall'altro che un rapporto proficuo tra profili e competenze diverse implica responsabilità e rispetto.

Il tema che il convegno di Napoli ha messo al centro della nostra attenzione richiede, in conclusione, una messa a fuoco complessiva e una impegnativa riflessione sul metodo, anzi sui metodi da sviluppare e approfondire per 'far parlare' le nostre fonti. Una sfida impegnativa che implica da un lato 'chiarire posizioni e metodologie', dall'altro 'affacciare anche qualche modello alternativo', in coerenza con le sollecitazioni che Armando Petrucci già trent'anni fa rivolgeva alle nostre istituzioni e ai nostri professionisti. 


\section{Bibliografia}

Federici, C. (1996). «Si tutela esponendo?». Leonardi, C. (a cura di), Segreti in vetrina. Utilità e danno per la storia delle mostre di libri, documenti e cimeli. Firenze: Fondazione Ezio Franceschini, 39-49.

Feliciati, P.; Alfier, A. (2013). «Archives on the Web and Users Expectations: Towards a Convergence with Digital Libraries». Review of the National Center for Digitization, 22, 81-92. http://elib.mi.sanu.ac.rs/files/ journals/ncd/22/ncd22081.pdf.

Feliciati, P.; Alfier, A. (2017). «Gli archivi online per gli utenti: premesse per un modello di gestione della qualità» JLIS.it, 8(1), 22-38. http://doi. org/10.4403/jlis.it-12269.

Giannini, F. (2015). «Un approccio idiota all'arte: a proposito delle mostre blockbuster». Finestre sull'arte. https://www.finestresullarte.info/309n_ mostre-blockbuster-approccio-idiota-all-arte.php.

MiBACT, Ministero per i beni e le attività culturali (1999). Conferenza nazionale degli archivi (Roma, Archivio Centrale dello Stato, 1-3 luglio 1998). Roma: Ministero per i beni e le attività culturali. http://www. archivi.beniculturali.it/dga/uploads/documents/Saggi_50.pdf.

Olla Repetto, G. (1996). «Il perché di una mostra». Gentium memoria archiva. Il tesoro degli archivi = Catalogo della mostra (Roma, Museo nazionale di Castel Sant'Angelo, 24 gennaio-24 aprile 1996). Roma: De Luca, 1-8.

Parenti, A.B. (1995). «"I segreti in vetrina”. Utilità e danno per la storia delle mostre dei documenti, libri e cimeli». Archivio storico italiano, 1, 151-8.

Petrucci, A. (1982). «Considerazioni impolitiche sul 'mostrismo'». Quaderni storici, 17, 51(3), 1159-64.

Salmini, C. (2014). «Gli archivi tra comunicazione e rimozione». Giuva, L.; Guercio, M. (a cura di), Archivistica. Teorie, metodi e pratiche. Roma: Carocci, 337-56. 



\title{
Etica della collaborazione e responsabilità Per una nuova gestione, valorizzazione e trasmissione del patrimonio culturale
}

\author{
Giorgio Busetto \\ Fondazione Ugo e Olga Levi Onlus, Venezia, Italia
}

\begin{abstract}
Proper management of institutional services and enhancement of cultural heritage can be achieved taking into account problems we face everyday. Throughout our activities we make choices that must be shared with all professionals involved in the complex management of cultural institution and heritage. The exhibitions - if wellconceived - represent a unique opportunity to enhance our heritage and disseminate knowledge by adopting new technologies.
\end{abstract}

Keywords Ethics of collaboration. Management. Cultural heritage enhancement. Library. Archives. Preservation. Exhibition.

Voglio partire dalle suggestioni avute dagli interventi di questa giornata e in particolare da una cosa detta da Mariella Guercio, da un suo preciso richiamo all'etica della collaborazione. La parola etica mi pare sia assolutamente centrale e mandi alla base di tutti i nostri problemi e anche di tutte quelle che potrebbero essere le nostre soluzioni. Su questo, e su tutti gli attaccamenti che abbiamo, di tipo identitario, di tipo professionale, bisognerebbe riuscire a lavorare per garantire la collaborazione, perché la collaborazione è resa spesso difficile dalle specificità e dal fatto che ognuno ritiene che le proprie specificità siano intoccabili.

Abbiamo visto con la globalizzazione cosa è accaduto ai principi: tante cose che noi credevamo essere principi si sono rivelate regole, perché non ave- 
vano la capacità di essere universali. E le regole devono essere conosciute, devono essere adoperate e nell'adoperarle devono poter essere violate, con molta tranquillità e molto senso di responsabilità.

Tanto tempo fa, quando dirigevo la Fondazione Querini Stampalia, mi ricordo che per un certo periodo avendo dei restauri in Museo avevo dei problemi con la tavola di Giovanni Bellini La presentazione al Tempio, quella che recentemente è stata esposta con una sorta di gemello o di antigrafo del Mantegna: una mostra itinerante, allestita a Venezia, a Londra e a Berlino (Blass-Simmen et al. 2018). Quel quadro poteva valere come tutto il Palazzo, un compendio di seimila metri quadrati. Stavamo inserendo tutte le misure di prevenzione incendi, costosissime. Mi ricordo di avere pensato: ma se dovesse prendere fuoco il Palazzo io dovrei preoccuparmi di salvare prima gli utenti della Biblioteca o il Bellini? Mi sono posto molto tranquillamente questo problema, nella certezza che il Bellini valeva più di molti utenti della Biblioteca da un certo punto di vista, ma che da un altro ero costretto ad ammettere che gli utenti della Biblioteca avevano la precedenza.

Non solo. La gestione del servizio, che è ovviamente cosa di grande complessità e che richiede ragionamenti importanti, ragionamenti di base e poi applicazione dei ragionamenti di base, comportava delle scelte sul funzionamento della Biblioteca. Per esempio, dopo gli ultimi importanti restauri che avevamo fatto con Mario Botta (Gemin 2015), non avevo fatto mettere la porta in Biblioteca pensando: la Fondazione è una struttura che ha Biblioteca, Museo e Manifestazioni culturali; devono essere considerate un insieme, quindi metto in Biblioteca tutti i quadri possibili togliendoli dai depositi, anche se le condizioni microclimatiche non sono ideali, perché comunque il fatto di poterli vedere ai fini conservativi è meglio che lasciarli nei depositi. Questo me lo aveva insegnato Francesco Valcanover, un non dimenticato sovrintendente veneziano, che mi aveva raccontato di avere una volta estratto una rastrelliera in un deposito e di avere visto i pezzi di colore che cadevano da un dipinto e quindi mi aveva raccomandato che si potessero continuamente osservare le opere per garantirne la conservazione. Così chi lo avesse voluto poteva visitare anche la Biblioteca, le cui belle sale fine Ottocento - primo Novecento ${ }^{1}$ erano arredate con molti dipinti.

Quando io ho lasciato l'istituto, dopo di me ha assunto la direzione una bibliotecaria. La prima cosa che ha fatto è stata far mettere la porta, con la motivazione che altrimenti, se ci fosse stato del pubblico estraneo alla Biblioteca avrebbe disturbato l'utenza della Biblioteca. Con la successiva direzione, sono stati addirittura messi dei tornel-

1 I restauri accennati non avevano toccato le sale a suo tempo sistemate dal direttore Giuseppe Mazzariol. Cf. Busetto 1992, 15-21. 
li, perché si voleva avere la certezza che il numero dei presenti corrispondesse e non superasse il numero stabilito dai Vigili del Fuoco.

Io ero molto più vago, non mi attenevo strettamente alla norma, facendo una serie di ragionamenti sulla circolazione dell'utenza all'interno della struttura. Molta utenza era studentesca, quindi prediligeva lunghe soste alla Caffetteria al piano terra lasciando il posto nelle sale di lettura al primo piano. Perciò non mi davo più di tanto preoccupazione della certezza dei numeri, pensando che comunque ragionevolmente si rimaneva nei limiti previsti: l'esatto numero corrispondeva al numero dei posti a sedere, e quindi era controllabile lateralmente, come si dice in lessico organizzativo, cioè era l'utenza stessa che si autoregolava, né le visite erano così frequenti da alterare tale equilibrio. Tuttavia mi ponevo il problema, pensando «il giorno che succede l'incidente e muore qualcuno, cosa dico io ai familiari di questo qualcuno che mi chiedono: perché tu hai violato la norma?» Per me questi sono problemi di natura etica che uno affronta quando ha la responsabilità della gestione.

Nei problemi di natura etica gioca un ruolo fondamentale la consapevolezza, che non è mai abbastanza, nel senso che non riusciamo a essere consapevoli di tutto, e la complessità più volte evocata oggi è il certificato che non siamo mai consapevoli di tutto.

Una delle consapevolezze che secondo me è importante è che conservare è selezionare. Noi abbiamo un patrimonio immenso che è impossibile conservare. Perché fosse possibile conservarlo bisognerebbe che tutti i cittadini della Repubblica italiana fossero investiti del compito consapevole della conservazione, ma così come stanno le cose bisogna che ci rassegniamo all'idea che è più importante la selezione della conservazione, senza la quale non si può arrivare alla conservazione stessa. È un drammatico dilemma, se volete, ma lo viviamo quotidianamente nei nostri istituti, volere o non volere. Il tentativo di conservare di più di quello che siamo in grado di conservare è disastroso per tutto ciò che conserviamo, quindi è anche importante porsi il problema di come affrontare la selezione e le conseguenze della selezione. Può essere che si selezioni e che dei materiali che non possono essere conservati da noi possano essere conservati da altri, con la formula del deposito, revocabile o perpetuo, o della cessione non onerosa: questa è un'altra questione interessante, che attiene inoltre all'ambito della cooperazione.

Tanti anni fa a me è capitato di occuparmi a lungo di biblioteche scolastiche e mi ricordo che ero arrivato all'idea che fosse preferibile eliminare le biblioteche scolastiche, ma che la condizione per eliminarle era che ci fosse un servizio di pubblica lettura capace di sostituire le biblioteche scolastiche anche entrando nelle scuole, cosa che naturalmente non siamo in grado di fare. Peraltro è certissimo che la biblioteca scolastica oggi per come è fatta è un puro e semplice spreco di risorse: di spazio, di patrimonio e così via. Naturalmente ci 
saranno le eccezioni. Io parlo sempre per generalizzazioni, e questo infastidisce spesso chi non si riconosce nella generalizzazione, ma la generalizzazione è fondamentale per porre sul tavolo le questioni.

Oggi abbiamo parlato molto di mostre e 'mostrismo', il che mi richiama subito alla mente la parola effimero.

Sono dibattiti che si agitano da decenni, ma che hanno caratterizzato soprattutto gli anni Settanta. Credo sia importante anche qui introdurre dei distinguo e delle valutazioni. L'effimero di Nicolini a Roma è stato un fatto di grande portata politica che ha rimesso i romani per la strada, in un mondo che era disastrato dal terrorismo, dal vandalismo e così via (Lipperini 1982; Nicolini 1991, 2002). Quell'effimero era un effimero sensato. È stato scimmiottato da una banda di assessori alla cultura degli enti locali che non sapevano quello che facevano e credevano di fare altro rispetto a quello che facevano. È sempre molto importante la contestualizzazione delle azioni, e questo di nuovo rinvia alla consapevolezza.

Il 'mostrismo' probabilmente c'è sempre stato. Recentemente ho dovuto lavorare su un carteggio di Carlo Ludovico Ragghianti che non le risparmiava mai a nessuno, ma certamente alcuni suoi giudizi su certi aspetti di alcune mostre sono molto interessanti e rinviano a questo genere di problematica (Busetto 2016). ${ }^{2}$

Un libro non recentissimo di Montanari e Trione si intitola Contro le mostre, ma nel libro non c'è, assolutamente un partito preso contro le mostre, ma la richiesta di avere delle buone mostre (Montanari, Trione 2017; Ferraresi 2017).

Nell'ultimissimo periodo ho fatto un'esperienza di mostra che vorrei definire addirittura tragica, perché mi sono imbattuto in un'alleanza fra dilettanti - armata Brancaleone - e delinquenti - banda Bassotti. L'insieme mi ha generato una tale quantità di problemi, che ancora non ne sono uscito. In quasi 50 anni di lavoro non mi ero mai imbattuto in una simile situazione. C'era un indice, però, che doveva allarmarmi e di cui non ho tenuto debito conto: quella mostra non aveva un comitato scientifico e, a richiesta, fatta al curatore, del comitato scientifico, questi si era opposto; poi si è capito nel passare del tempo quanto fosse importante per lui non avere un comitato scientifico per operare con questa mostra con delle finalità assolutamente inappropriate.

Le mostre si possono e si debbono fare, ma vanno fatte in maniera appropriata, con un progetto adeguato, con un professionismo adeguato, con una molteplicità di strumenti. Io ho avuto delle esperienze molto felici quando dirigevo la Querini Stampalia, con dei progetti espositivi che partivano dal restauro delle opere, che mi hanno

2 Ragghianti ha attività sterminate, anche nel campo delle mostre; cf. anche Massa, Pontelli 2018. 
consentito poi di commercializzare il lavoro che ho fatto, in Italia e all'estero, generando delle entrate importanti per la vita del Museo. Ma questo nasceva da un progetto che metteva alla base il restauro, e allineava le attività degli storici accanto agli storici dell'arte, faceva sviluppare anche una ricerca archivistica importante, un lavoro iconologico importante, iconografico importante, coinvolgendo anche le risorse interne dell'Istituto, in modo che tante diverse persone si trovassero a studiare, cosa che da noi non si fa mai, o si fa troppo poco, perché il peso delle attività quotidiane, le cosiddette attività riproduttive, ${ }^{3}$ è oppressivo. Abbiamo visto così che dedicare tempo allo studio consente di realizzare un catalogo che oltre a saggi di studio, sia ricco di schede e apparati informativi capaci di aiutare a capire. ${ }^{4}$

Evidentemente ci sono delle necessità. Per esempio quando io visito un museo archeologico mi trovo in presenza di cumuli di rovine, di pezzi di cose che non mi dicono più di tanto. Quando visito lo stesso posto con un archeologo si dischiude un mondo. Allora il problema quale è? Il problema è di riuscire ogni volta a far vivere, a far parlare ciò che possediamo, ciò che riteniamo debba essere oggetto di attenzione richiamata da parte di un pubblico più o meno vasto, perché non necessariamente dobbiamo andare in cerca dei grandi numeri, ma dobbiamo andare in cerca dei numeri giusti, cioè del livello appropriato di destinazione di ciò che facciamo. ${ }^{5}$

Quindi nella valorizzazione, per esempio, dei fondi, anche dei fondi di persona, la mostra è certamente importante, e io vedo ancora più importante la mostra virtuale, perché consente una esplorazione più approfondita del fondo, e se possibile i seminari sul fondo, evidentemente per un pubblico più di addetti ai lavori che per un pubblico largo. D’altra parte la valorizzazione, come è stato detto, è prima di tutto rendere disponibili i materiali, quindi si traduce nella corretta conservazione, nell'inventariazione, nella catalogazione. Pensate cosa vuol dire allestire una sala di consultazione, mantenere l'aggiornamento di una sala di consultazione: sono tutte operazioni che

3 Il bibliotecario è come una casalinga, deve rimettere a posto ogni giorno il disordine che fanno gli utenti e infatti ci sono molti bibliotecari che odiano gli utenti e non sono i migliori bibliotecari, perché l'utente deve mettere in disordine e va punito quando cerca di mettere in ordine i libri, perché non li deve ricollocare.

4 Ricordo ancora la soddisfazione provata quando una collega, redigendo le schede di una coppia di dipinti del Padovanino, ha illustrato i cartigli, rilevandovi il verso di un salmo, arrivando così a capire che un cartiglio lacunoso era stato integrato erroneamente da un restauratore, che le tele in pendant erano state ridotte, che il soggetto iconografico (e quindi il titolo dell'opera) andava corretto, come pure la data. Cf. Busetto 2004, 142-4, schede nrr. 2 e 3 di Tiziana Bottecchia.

5 L'argomento è talmente significativo e avvertito che il Ministero Beni e Attività Culturali ha ritenuto opportuno dedicare un sito ai nuovi migliori allestimenti: http:// www.allestimentimuseali.beniculturali.it/index.php?it/1/home. 
costano, soprattutto in termini di risorse umane, e però sono indispensabili se si vuole dare un significato anche economico complessivo a ciò che si conserva.

Una delle grandi difficoltà che noi abbiamo oggi è quella del trapasso: noi viviamo in una età di mezzo tra uno stato del passato e una condizione del futuro. Autori di fantascienza e futurologi hanno disegnato una serie di scenari molto interessanti e pian piano ci stiamo avvicinando. Quando io ero bambino avevo gli albi per la raccolta delle figurine. Uno di questi era il 2000. Quello che c'era in quell'albo in parte si è realizzato e in parte invece dobbiamo ancora aspettare, ma certamente il 2000 è un momento importante, il fatto di essere entrati in questo terzo millennio non è poca cosa. Allora verso quale futuro stiamo andando? Il nostro trapasso da quello che c'era ieri, che conosciamo benissimo, a quello che sarà domani dovremmo però cercare di conoscerlo, perché ci sta cascando addosso comunque, che lo vogliamo o no.

La tecnologia ha uno sviluppo geometrico che non è coerente con lo sviluppo della cultura umana. Prima che si acculturi un uomo deve passare una generazione, mentre lo sviluppo tecnologico è immenso; è stata citata stamattina, per esempio, la diffusione del cellulare. Ormai si lavora con il computer. Io lavoro ancora con la penna e la carta, con la tastiera e lo schermo, però scrivo con i libri, tanti libri davanti, ma anche col computer. Io uso anche Wikipedia, non mi vergogno a dirlo; ne sono anche un modestissimo finanziatore, appartengo a quel due per cento degli utenti che contribuiscono, verso qualche euro perché ne riconosco l'utilità. Però anche qui noi abbiamo da una parte l'abbassamento della qualità, fatto generalizzato (Mantellini 2018), ${ }^{6}$ che secondo me non dobbiamo in nessun modo impedire o cercare di contrastare, dobbiamo prenderne atto e cercare di governare anche questo fenomeno. Io insegno all'università, a un livello richiestomi che è enormemente più basso di quello che era quando l'ho fatta io l'università; ma ci sarà una ragione per questo: in direzione di quale struttura delle competenze stiamo andando? A me sembra verso una struttura molto specialistica e anche tutto sommato alta nello specialismo e bassa appena si esce dallo specialismo. Con conseguenze non di poco conto. Quando lavoriamo ci accorgiamo che è importante il ricorso a competenze specialistiche, ma che è un costo rilevante anche questo, generalmente non è un costo ammesso nei nostri bilanci, eppure è un qualche cosa di indispensabile.

Ebbene, il futuro verso il quale andiamo è un futuro in cui accumulazione e trasmissione del sapere non avverranno come sono av-

6 La copertina parlante annuncia: «Dentro alla vastità dell'offerta digitale, tutto ci è sembrato a portata di mano. Proprio allora abbiamo deciso di rallentare, compiendo una scelta inattesa: la riduzione delle nostre aspettative. Capire questa scelta significa capire la contemporaneità». 
venute nei primi due millenni, avverranno in un'altra maniera. Arriverà tutto attraverso le macchine. Collegate tra loro, saranno capaci di assumere le conoscenze di ogni singola macchina e trasmetterle a tutte le macchine, saranno in grado di trasmettere la conoscenza accumulata attraverso il suono e l'immagine, fissa e in movimento, e tutto questo è molto più coerente col lavoro della mente, come ci insegna chi ha lavorato sul sogno, di quanto non lo sia la trasmissione attraverso la parola scritta. ${ }^{7}$

La parola scritta non cesserà per questo di esistere, ma verrà anche tradotta in suono, in parola detta.

Se noi pensiamo che questa è la prospettiva del trasferimento, se dobbiamo fare i conti con questo processo, dobbiamo cercare di accompagnarlo e di regolare il nostro lavoro in modo tale che tutto ciò possa avvenire nel migliore modo possibile, nel più sensato modo possibile.

Mi ricordo, per esempio, una polemica che ho avuto anni fa all'interno del mio istituto: avevamo destinato delle risorse importanti al centenario di due autori, risorse in parte proprie e in parte di provenienza regionale - quando ancora la Regione Veneto era contributiva - ma non siamo riusciti a fare le voci di Wikipedia di quei due musicisti. Io non mi dovevo occupare di queste cose perché non era di mia competenza, ma ho molto protestato per questo; ma se chi dirige è un professore universitario è scarsamente interessato a questo tipo di ricaduta, che invece è importante. ${ }^{8}$

Quindi noi dobbiamo tenere conto di tante questioni diverse.

Una delle questioni poste è stata quella del possibile incremento o mancato incremento dell'utenza da parte del digitale. Bene, io ho visto questo a Bologna, al Martini, dove il grande lavoro fatto sul web - un bellissimo lavoro - ha moltiplicato l'utenza da remoto e ha diminuito quella in presenza. Alla Fondazione Levi sta accadendo progressivamente la stessa cosa: sempre di più l'utenza da remoto si moltiplica e sempre di più si sta cercando di allestire una base di dati dove entri tutto quello che ha fatto la Fondazione. Perciò è im-

7 Tra mitologia, letteratura, antropologia, neuroscienze, e così via, la bibliografia sul sogno è sterminata. Il riferimento capitale rimane per me Freud L'interpretazione dei sogni ([1899] 2018), testo fondativo della psicanalisi che è forse la disciplina che più ha lavorato sul sogno; diversa (secondo me molto pasticciata) la concezione di Carl Gustav Jung, che come Freud interviene a più riprese sul sogno, in particolare con L'applicabilità pratica dell'analisi dei sogni del 1931 e L'essenza dei sogni del 1948, anno in cui rimette mano alle Considerazioni generali sulla psicologia del sogno del 1916; si veda la recente messa a punto di Vitolo 2019; difficile ma straordinaria la riflessione di Jacques Lacan, incredibile lettore di Freud (Lacan 1955, 2013), soprattutto con gli inediti Seminari, non ancora tutti tradotti in italiano; cf. il riordino bibliografico in https:// it.wikipedia.org/wiki/Opere_di_Jacques_Lacan. Rilevo che il XII Congresso mondiale dei lacaniani, che si terrà a dicembre a Buenos Aires, è puntualmente dedicato a Il sogno. La sua interpretazione e il suo uso nella cura lacaniana.

8 Con assai maggiore dottrina della mia, condivide questa opinione Bianchini 2019, 156. 
portante che anche l'archivio della Fondazione, soprattutto per certe sezioni, entri a far parte di questo database e che tutto quanto quello che appartiene ormai alla storia della Fondazione sia navigabile e ricercabile: è il grande tema dell'irruzione del digitale.

Si è parlato qui anche della debolezza del Ministero Beni e Attività Culturali e Turismo e della necessità che tale Ministero abbia i migliori professionisti, ma questo dovrebbe essere una necessità di tutti dovunque e comunque, non solo del Ministero-guida. Per esempio quando io ho preso in mano l'Archivio della Biennale ho visto delle cose incredibili in termini di sprechi a partire proprio dalla mancanza di adeguamento a quelle che erano delle linee guida degli Istituti centrali del Ministero; d'altra parte non esistevano strumenti, cioè lo Stato elargiva dei soldi ma non esercitava né indicazioni né controlli sull'uso di questi fondi e sul conseguente spreco. All'università avveniva la stessa cosa. Anche se l'università dovrebbe avere i migliori professionisti, temo che non sia sempre così, soprattutto che non intenda creare i migliori professionisti. Noi abbiamo tutta una serie di problemi in questo paese, ricchissimo di risorse umane e materiali di ogni genere, ma poverissimo di capacità di governo a tutti i livelli, da quello individuale a quello nazionale. Sono temi che ribaltano nuovamente nell'etica e con questo torniamo alle considerazioni iniziali, alla questione che sta alla base di tutti i nostri problemi e anche di tutte quelle che potrebbero essere le nostre soluzioni. ${ }^{9}$

\section{Bibliografia}

Aurigemma, L. (a cura di) (2016). Carl Gustav Jung: Opere. Torino: Bollati Boringhieri.

Bianchini, C. (2019). «Il lavoro sulle collezioni della Fondazione Levi». Busetto, G. (a cura di), Biblioteca Gianni Milner 2012-2022, 1, 156.

Blass-Simmen, B.; Rowley, N.; Villa, G.C.F. (a cura di) (2018). Bellini / Mantegna. Capolavori a confronto. Presentazione di Gesù al Tempio = Catalogo della mostra (Venezia, Museo della Fondazione Querini Stampalia, 21 marzo-1 luglio 2018). Cinisello Balsamo: Silvana.

Busetto, G. (1992). "Mazzariol alla Querini». Bertola, C. (a cura di), Giuseppe Mazzariol. 50 artisti a Venezia. Milano: Electa, 15-21.

Busetto, G. (a cura di) (2004). Dei ed eroi del barocco veneziano. Dal Padovanino a Luca Giordano e Sebastiano Ricci. Catania: Maimone.

9 Barbara Poli, che ringrazio per la prima lettura del testo di trascrizione, opportunamente commenta: «Sul piano dei contenuti, mi pare che accanto al concetto di etica della collaborazione dovrebbe essere messo di più in rilievo il concetto di 'capacità di governo', che appare solo di sfuggita, e anche quello di visione d'insieme, o di organizzazione delle funzioni e delle competenze, perché da tutto il discorso appare chiaro che manca una visione complessiva e organizzata;non vorrei insomma che 'etica della collaborazione' fosse intesa solo come un dovere individuale, quasi di buona volontà, e non un dato di sistema frutto di un pensiero complesso e articolato». 
Busetto, G. (2016). «Lettere fra professori: il carteggio Ragghianti-Mazzariol». Luk, n.s., 22, 55-9.

Ferraresi, G. (2017). Contro le mostre. La retorica della bellezza, i capolavori feticcio, il patrimonio stabile ignorato: Tomaso Montanari racconta tutto quello che non va nel mondo dell'arte in Italia. Luz. https:// luz.it/spns_article/intervista-tomaso-montanari/.

Freud, S. [1899] (2018). L'interpretazione dei sogni. Musatti 2018, 3.

Gemin, M. (a cura di) (2015). Mario Botta Querini Stampalia. Fotografie di A. Chemollo. Pordenone: Giavedoni.

Jung, C.G. [1916-48] (2016). Considerazioni generali sulla psicologia del sogno. Aurigemma 2016, 8: 254-99.

Jung, C.G. [1931-34] (2016). L'applicabilità pratica dell'analisi dei sogni. Aurigemma 2016, 16: 149-72.

Jung, C.G. [1945-48] (2016). L'essenza dei sogni. Aurigemma 2016, 8: 301-20.

Lacan, J. (1955). Scritti. 2 voll. Torino: Einaudi.

Lacan, J. (2013). Altri scritti. Testi riuniti da J.-A. Miller; edizione italiana a cura di A. Di Ciaccia. Torino: Einaudi.

Lipperini, L. (1982). «Cronache dal Palazzo d'Inverno». Sipario, 37(416), 99-102.

Mantellini, M. (2018). Bassa risoluzione. Torino: Einaudi.

Massa, S.; Pontelli, E. (a cura di) (2018). «Mostre permanenti». Carlo Ludovico Ragghianti in un secolo di esposizioni. Lucca: Fondazione Ragghianti.

Montanari, T.; Trione, V. (2017). Contro le mostre. Torino: Einaudi.

Musatti, C.L. (a cura di) (2018). Sigmund Freud: Opere. Torino: Bollati Boringhieri.

Nicolini, R. (1991). Estate romana. Siena: Sisifo.

Nicolini, R. (2002). «Strategia della cultura contro strategia della paura». Lo spettacolo, 52(1-2), 69-75.

Vitolo, A. (2019). «Carl Gustav Jung: l'immagine onirica, la prospettiva ermeneutica». Musella, R.; Trapanese, G. (a cura di), L'interpretazione dei sogni. Dialoghi sulla tecnica psicoanalitica. Milano: FrancoAngeli, 175-88. 



\title{
Non di sole mostre vivono gli archivi
}

Micaela Procaccia

Associazione Nazionale Archivistica Italiana - ANAI, Roma, Italia

\begin{abstract}
The current phenomenon of the proliferation of exhibitions presenting different pieces of cultural heritage, sometimes with considerable public success, affects the archives sector in a modest way. Historical documents are intended for the study of researchers and largely not aesthetically attractive to the general public and effectively communicated in slower and more complexways: in-depth educational initiatives, web portals that provide information on the historical and documentary context, dedicated TV programs. The importance of historical memory and cultural heritage as a whole, as a factor of identity and civil conscience was underlined by the experience of the coronavirus pandemic.
\end{abstract}

Keywords Archives. Documents. Exhibitions. Education. Identity.

Le riflessioni che seguono riflettono quanto detto in occasione della tavola rotonda conclusiva della giornata Oltre le mostre. Proposte per una diversa valorizzazione del patrimonio archivistico e librario tenutasi a Napoli il 28 febbraio 2020. Tuttavia è impossibile prescindere, scrivendo a oltre un mese di distanza, da alcune considerazioni scaturite dalla fase di emergenza, ancora in corso, derivata dalla pandemia del virus Covid-19 e che concluderanno il testo.

Nella bella giornata napoletana, appena agli inizi della diffusione del virus e ancora in un clima di non completa consapevolezza della sua portata, ero partita da alcune constatazioni che ancora oggi ritengo valide. Innanzi tutto, il fatto che la diffusa malattia del 'mostrismo', per citare una espressione più volte utilizzata nel corso della giornata (Petrucci 1982a) trova qualche difficoltà a contagiare il mondo degli archivi. Per una serie di motivi strettamente collegati alla banalizzazione culturale tipica del 'mostrismo' e alla peculiarità del bene archivistico. 
Il 'mostrismo', infatti, considerato come una variante di approccio superficiale al patrimonio culturale, è un fenomeno caratterizzato dalla compresenza di grandi numeri di visitatori con modalità veloci di fruizione delle esposizioni e una contaminazione con forme di presenzialismo e mondanità.

I documenti storici spesso non sono belli, benché importanti e la loro rilevanza storica non ha a che fare con il loro aspetto esteriore, anche se esistono documenti archivistici corredati di miniature, immagini e altri tipi di decorazione di grande bellezza. Si pensi alle Biccherne senesi, documenti contabili rilegati con copertine lignee dipinte di notevole valore artistico che, non a caso, sono state oggetto di diversi trafugamenti e sparizioni, distaccate dal loro contesto documentario che, come tutte le annotazioni contabili non è particolarmente suggestivo per l'occhio profano.

In una mostra dedicata ai 500 anni dalla morte di Raffaello, recentemente inaugurata (con una certa fretta, occorre dire) e poi forzatamente chiusa a causa del diffondersi del coronavirus, è esposto un documento di enorme importanza ma decisamente poco attraente dal punto di vista estetico, anzi sostanzialmente illeggibile per chi non è un paleografo, pieno di correzioni, interventi e via dicendo. Si tratta della famosa lettera di Raffaello e Baldassarre Castiglione al pontefice Leone $\mathrm{X}$ nella quale si introduce il concetto di tutela del patrimonio archeologico, artistico e storico (in particolare, delle rovine di Roma). Ora, affinché il visitatore, trasportato attraverso una passerella di opere che, bene o male, anche se non particolarmente versato in storia dell'arte è in grado di riconoscere superficialmente come belle e significative, per queste pagine (poco più di foglietti) disordinate e incomprensibili, ha bisogno di spiegazioni, contesto storico e cognizioni che gliene facciano comprendere l'importanza, pari a quella dei dipinti che attraverso riproduzioni, commercializzazioni e quant'altro ha imparato a riconoscere.

E così è, in generale, per tutti i materiali conservati negli archivi (salvo, come si è detto, le non predominanti eccezioni), ragione per cui allestire una mostra archivistica presenta esigenze complesse e diverse rispetto ad altre mostre, in parte simili, ma in maniera non del tutto coincidente, ai problemi posti da una mostra libraria, spesso ben più ricca di materiali visivamente 'preziosi' di quanto non siano i materiali archivistici.

La capacità di spiegare il contesto storico e di produzione del documento, di renderlo fruibile ove occorra attraverso trascrizioni, spiegarne autori e destinatari, il tutto in maniera comunicativa e non specialistica ma senza cedere alla banalizzazione, sono solo alcuni degli ostacoli che chi allestisce una mostra documentaria si trova ad affrontare. Insieme a quello più importante, ovvero il fatto che per statuto i documenti archivistici servono per ricostruire la storia in modo scientifico e approfondito e l'esposizione come una sorta di feticcio è del tutto 
estranea alla loro natura. Vanno soprattutto studiati, più che esposti. Tutto ciò non significa che non si possano fare mostre con il patrimonio conservato negli archivi. Lo stesso Armando Petrucci, che ha scritto:

il 'mostrismo' forzato a ritmi frenetici nasconde l'incapacità da parte dei gestori delle strutture pubbliche di programmare e di realizzare una politica culturale di lunga durata, tesa ad affrontare e risolvere i nodi reali della conservazione, della conoscenza, dell'uso del nostro patrimonio storico-artistico. (Petrucci 1982a, 1159)

curò una mostra documentaria assolutamente esemplare e di grande successo intitolata Scrittura e popolo nella Roma barocca 1585-1721.

E tuttavia, è evidente che la valorizzazione dei documenti d'archivio non vede nelle mostre il suo veicolo principale. L'esperienza dimostra che le strade sono altre.

Un fenomeno che colpisce è il fatto che non appena una sezione di Archivio di Stato, ovvero quelle porzioni di istituto collocate per ragioni storiche in una cittadina diversa dal capoluogo di provincia istituzionalmente sede dell'Archivio di Stato, rischia di essere chiusa per ragioni di contenimento della spesa, si scatenano le proteste in sede locale. Per tacere del malumore delle comunità quando l'archivio storico comunale, a seguito di una catastrofe come un terremoto, viene recuperato dalle macerie e ricoverato in una località diversa. Queste comunità, queste popolazioni, in entrambi i casi lamentano la perdita della loro memoria, del patrimonio identitario locale. Non è detto (anzi, raramente, ciò accade) che in precedenza negli stessi luoghi si fosse prestata una grande attenzione all'archivio storico. $\mathrm{Ma}$, come è noto, è quando si rischia di perdere qualcosa che si comincia a riconoscerne l'importanza. L'esperienza contraria ma coincidente è quella che avviene quando un intervento di tutela positivamente realizzato (un riordinamento, un recupero) viene condiviso in qualche modo con la cittadinanza. I colleghi bellunesi mi hanno raccontato che quando i documenti del processo per il disastro del Vajont, conservati nell'Archivio di Stato de L'Aquila, città sede del processo, furono portati a Belluno per essere digitalizzati, la gente si fermava lungo la strada per veder passare i veicoli che li trasportavano. Così come l'entusiasmo degli studenti coinvolti nelle numerose iniziative di alternanza scuola-lavoro negli Archivi di Stato, condotti attraverso percorsi di conoscenza della storia della loro comunità mediante lo studio dei documenti, testimonia di quanto sia inattendibile il luogo comune che vuole gli archivi poco attraenti per un grande pubblico.

Gli archivi vanno semplicemente comunicati in modo più complesso, più approfondito, se vogliamo più lento di quello ridotto ai pochi minuti di visione concessi nell'ambito di una mostra con migliaia di visitatori. Gli archivi sono uno Slow Food, efficaci se la comunicazione è meditata e qualitativamente alta. 
Cosa che non esclude livelli diversi: anni fa, una intelligente direttrice di Archivio di Stato usava organizzare una caccia al tesoro di documenti d'archivio (ovviamente riprodotti) fra ragazzini delle scuole della città che si concludeva con il privilegio per la squadra vincitrice di contemplare un documento medievale originale. Era sempre un grande successo. Allo stesso modo, centinaia di alunni di quinta elementare hanno ascoltato con grandissima attenzione una lezione sulla Costituzione all'Archivio centrale dello Stato, potendo avere accesso alla sua copia anastatica, che questi giovanissimi, al termine delle spiegazioni hanno osservato con palese emozione.

Uno strumento che ha dimostrato una sua validità, purché realizzato con la cura per la descrizione dei contesti storici e archivistici di cui prima si diceva e con un approfondito studio preliminare, è quello dei portali web. Il mondo della rete è relativamente ricco di documenti digitalizzati e pubblicati, anche su portali o anche su pagine Facebook di singoli o istituzioni, ma molto spesso si tratta di documenti semplicemente scansionati e caricati, senza alcuna informazione di corredo o con minimi dati esplicativi, del tutto insufficienti a rendere adeguatamente utilizzabili le informazioni contenute nel documento da parte di un pubblico generico, sebbene talvolta genericamente colto. L'Amministrazione archivistica si è sforzata di creare portali che contemperassero il rigore scientifico delle informazioni con lo sforzo di comunicarle a più livelli. Un obiettivo non semplice da raggiungere e molto ambizioso che ha incontrato successo soprattutto nei casi in cui ha intercettato un bisogno concreto degli utenti del web o una curiosità culturale diffusa. Emblematico è il caso dei milioni di contatti sul portale Antenati ${ }^{1}$ dedicato alla pubblicazione (ma non solo) dei registri di stato civile fino a cento anni fa e alla ricerca anagrafica. E altrettanto importante è stato il successo di una serie di trasmissioni di approfondimento dedicate agli archivi sul canale Rai Storia.

E per quanto riguarda l'immediato presente, un bell'articolo pubblicato online da un archivista, Leonardo Musci, mi suggerisce quelle considerazioni aggiornate di cui parlavo all'inizio. Scrive Musci:

Per assurdo che possa sembrare siamo ora quasi costretti a riscoprire il valore lenitivo dei depositi di memoria. Ė sorprendente come in questi giorni di lentezza e quiete [...] gli archivi digitali diventino la miniera dove attingere stimoli culturali o di svago per riempire il vuoto

Chi fa cultura mette online le sue produzioni, spettacoli teatrali o concerti, i musei si attrezzano con visite guidate virtuali, le biblioteche promuovono il prestito di e-book, gli archivi stori-

1 http://www.antenati.san.beniculturali.it/. 
ci mostrano i loro tesori, le radio (viva Radio3!) invitano a scaricare vecchie trasmissioni di tutti i tipi, la televisione pesca nelle sue teche le cose migliori dall'intrattenimento, alla comicità, allo sport. Se non possiamo produrre o lo possiamo fare a ritmo ridotto, compensiamo il meno presente con più passato, forse anche perché percepiamo, con maggiore o minore consapevolezza, che quello che ci accomuna non è solo una bandiera o un inno ma i nostri romanzi di formazione, le canzoni che ci hanno emozionato, le passioni che ci hanno diviso. (Musci 2020)

Aggiungerei che in un momento in cui la tenuta della società nel suo complesso, messa a dura prova dai provvedimenti che inducono tutti a una forzata chiusura a casa, si realizza in larga misura sui temi dell'identità e del riconoscimento di sé stessi come parte di un tessuto civile e comunitario, il valore della memoria diventa centrale e con essa di tutto l'insieme di patrimonio culturale che conserva e costituisce questa memoria e queste identità. Non a caso sui canali delle chat si susseguono video autoprodotti, montaggi, canzoni rivisitate, che mettono insieme la 'grande bellezza' (termine quanto mai abusato) con scene di Roma città aperta e anche documenti messi quotidianamente online da numerosi archivi che collezionano migliaia di like, forse insperati, per le loro iniziative. Il richiamo al passato, in forma digitale, è parte della nostra attuale capacità di resilienza ma occorre tenere ben presente il rischio ad esso connesso ed essere consapevoli che se «il digitale riempie il tempo" può anche produrre "spazzatura informativa che si propaga velocemente» (Musci 2020). Ciò significa che, al tempo della pandemia, le istituzioni che custodiscono la memoria (che siano archivi tradizionali e digitali, biblioteche, musei, edifici, siti archeologici) meritano una attenzione particolare, come la meritano tutti i professionisti del settore.

Quando dovremo pensare il tempo dopo la pandemia (e il momento è adesso) occorrerà investire nella tutela del patrimonio, nel reclutamento dei professionisti, nella progettazione della comunicazione, ricordando il ruolo strategico degli archivi nella costruzione di quel sentimento civile di appartenenza alla comunità che oggi sta tenendo insieme la società italiana non meno degli altri settori della cultura, ma anzi, sostenendoli col suo patrimonio di informazioni.

La lezione di questi giorni ci richiama all'importanza della sanità pubblica, dell'istruzione, della tutela dell'ambiente, ma anche al fatto che sono la memoria e la storia comuni, fatte di patrimonio culturale, storie di famiglia e dei mille borghi e città, paesaggi urbani ed extraurbani condivisi, che stanno tenendo in casa i cittadini e li stanno facendo sentire parte di una nazione.

Dimenticarsi di questo, passata la paura, e non considerare prioritario investire nella custodia di questa memoria e di questa storia, 
perpetuare la cattiva abitudine di tanti anni passati di considerare i beni culturali come semplici giacimenti da sfruttare per il turismo o come strumenti con cui:

il potere centrale e locale presenta sé stesso, lusinga e convince quello che considera il suo pubblico. (Petrucci 1982a, 1164)

sarebbe l'ennesima assurda miopia.

\section{Bibliografia}

Petrucci, A. (1982a). «Considerazioni impolitiche sul 'mostrismo'». Quaderni storici, 17(51), 1159-64. Poi in: Petrucci, A. (2019). Scritti civili. A cura di A. Bartoli Langeli, A. Ciaralli e M. Palma. Roma: Viella, 64-9.

Petrucci, A. (a cura di) (1982b). Scrittura e popolo nella Roma Barocca 15851721 = Catalogo della mostra (Roma, Palazzo Braschi, 1982). Roma: Quasar.

Musci, L. (2020). «Pandemia, memoria e consumo culturale». pagina21, 30 marzo. https://www.pagina21.eu/pandemia-memoria-consumo-culturale/leronardo-. 


\title{
L'Archivio della Pontificia Università Gregoriana (APUG) e la costruzione di un oltre
}

Martín M. Morales

Pontificia Università Gregoriana, Roma, Italia

\begin{abstract}
Within our social system the discourse about cultural 'objects' is strictly connected to the concept of enhancement, derived from the economical field as much as several other terms today often used to refer to these objects ('goods', 'consumption' etc.). Expositions and digitization are usually two examples of enhancement where the economical and consumer aspects are prevalent over the semiophoric dimension which marks out cultural objects. Besides proposing a reflection about these concepts, the paper presents the enhancement practices carried out by the Historical Archives of the Pontifical Gregorian University, with a particular focus on the web platform Gregorian Archives Texts Editing (GATE).
\end{abstract}

Keywords Digitization. Consumerism. Cultural industry. Enhancement of cultural heritage.

Sommario 1 Introduzione. - 2 Oltre le mostre. - 3 Oltre la digitalizzazione. - 4 Ciò che mostriamo.

\section{Introduzione}

Il seminario Oltre le mostre, organizzato da AICRAB (Associazione Italiana dei Conservatori e Restauratori degli Archivi e delle Biblioteche), è una occasione propizia per condividere e riflettere sull'interazione del patrimonio librario e archivistico con la complessità del sistema sociale. Riflessioni e pratiche possono essere concepite nella loro dimensione sistemica. Contro gli approcci teoretici spesso si muove una obiezione: qual è l'utilità di questa teoria? Come se la validità della teoria dipendesse dalla sua efficacia pratica. 
A dire il vero, questa correlazione stretta tra teoria e pratica, dalla quale dipenderebbe la sua efficacia, può essere datata nel XIX secolo, in contrapposizione, quindi, a un determinato concetto di scienza (si vedano le riflessioni di Luhmann 2005, 387-8). Un'alternativa a questa posizione è il rapporto che stabilì Boezio nel De Consolazione philosophiae (sec. V) che rappresenta la filosofia come una donna splendida e venerabile il cui vestito era ornato con due lettere $\Pi$ e $\Theta$, Praxis e Theoresis. Questa concezione si manterrà fino alla modernità incipiente.

La teoria riflette, oltre che su sé stessa, sulle pratiche; mantiene con loro un rapporto lasco e non dovrebbe essere limitata dalla poiesis. L'elaborazione teorica, per essere tale, non deve essere condizionata da nessun limite. La teoria si auto-valida in base alla propria capacità di porre domande e di problematizzare.

In primo luogo si dovrebbe riflettere riguardo l'aspirazione di superare una determinata pratica. Se si volesse procedere oltre si potrebbe pensare che si desidera andare verso un'alternativa a ciò che si è dato come stabilito, superare una linea osservata in quanto limite. Potrebbe essere un'occasione per valicare un problema, per oltrepassare un'aporia, o quanto meno per stabilire una nuova aspettativa. Ma il rischio, se non si determina con una certa precisione il punto di partenza, è di fare una rivoluzione nel senso originario della parola, vale a dire, tornare allo stadio precedente.

L'andare oltre in qualche modo suppone superare, ma allo stesso tempo la questione superata resta come un punto fermo che permette l'andare oltre. Se per qualche motivo, per esempio, si decidesse di valorizzare il patrimonio di un archivio o di una biblioteca andando oltre la pratica delle mostre e percorrendo alternative, questo non necessariamente implicherebbe aver compreso la problematica delle mostre e quindi il perché si dovrebbe andare al di là. Una insufficiente descrizione del problema difficilmente potrà assicurare alternative che siano tali. Come ricorda Aristotele nella sua Metafisica (III, 995 a 27-995 b 4), nessuno può sciogliere un nodo se non sa come farlo.

\section{Oltre le mostre}

Di solito, le mostre di libri e documenti di archivio, viste le caratteristiche strutturali e materiali degli oggetti esposti, si collocano nell'elenco delle cose da non fare e sono un messaggio contrario ai più elementari principi della conservazione. Malgrado ciò si continuano a proporre, come obbedendo a una determinata coazione a ripetere. Potremmo osservare questa ed altre iniziative in quanto comunicazioni all'interno del sistema sociale che rispondono a determinate aspettative. Vale a dire, non come azioni comunicative riferite a una determi- 
nata azione umana ma come le operazioni proprie del sistema sociale grazie alle quali esso si costituisce. Il nostro sistema sociale non segue più un ordine gerarchico (né di persone, né di valori) universalmente ammesso, ma è costituito da sistemi di comunicazioni tematicamente e funzionalmente orientati. Nella nostra società a nessun sistema (scienza, economia, politica, arte, religione, diritto, educazione), nemmeno al sistema di relazioni personali o familiari, può attribuirsi il dominio assoluto. Un sistema può farsi sentire con maggiore o minore potenza producendo eventualmente inattese risonanze e irritazioni negli altri ma senza la pretesa di causare cambiamento alcuno.

In questo senso, le mostre pensate all'interno del sistema sociale rimanderebbero a una semantica propria dell'economia la quale, nella concezione sistemica tratteggiata, non si orienta verso una funzione tendente a soddisfare dei bisogni, pur avendo questi un ruolo importante, giacché essi stessi sono determinati dall'economia. Potrebbe identificarsi, invece, la funzione specifica del sistema economia nella produzione e nella regolazione della scarsità, dinamismo questo sollecitato per la percezione di un futuro immediato. Quindi, libri antichi e documenti sono osservati come beni, e come beni che diventeranno scarsi nel tempo.

Questa concezione conoscerà una serie di declinazioni sempre in chiave economica. Così i beni culturali sono stati presentati come 'giacimenti culturali', dai quali si deve estrarre ricchezza e più recentemente sono stati offerti come 'cibo per la mente', associandoli al consumo e quindi alla produzione di ricchezza. Uno dei possibili corollari di questo tipo di comunicazione sarà quello di individuare nel profilo del manager il gestore di questi beni.

L'attribuzione di valore degli oggetti esposti spesso ha come cornice referenziale un mercato nel quale circolano o possono circolare. Questo riferimento potrà affacciarsi nel momento in cui si deve valutare la possibilità di intraprendere costosi interventi di conservazione o di restauro per attutire, nella misura del possibile, l'inesorabile passo del tempo. Diversamente, libri logorati dall'uso appaiono come un motivo ricorrente nelle rappresentazioni delle vanitas. Così, per il Maestro della Vanitas (sec. XVII), il libro, veicolo di un sapere effimero che era stato pagato ad un alto prezzo, è in compagnia del teschio che, come spiegato sul nastro che avvolge entrambi, è il vero testo che narra la discendenza di Adamo, intrisa di corruzione e di morte. Abbondanti testimonianze di questa ostentazione del libro come bene consunto associato alla caducità umana, possono trovarsi in altri maestri fiamminghi del XVII secolo come, De Heem, Claesz, Stoskopff, Steenwick, o Collier. Tra le lezioni moralizzanti di queste vanitas [fig. 1] il libro simboleggia la frivolezza dell'uomo che spende tempo e denaro per acquisire una conoscenza che davanti alla morte è futile ed evanescente.

Questa connotazione del libro valorizzato in quanto oggetto o per meglio dire in quanto cosa, si evolverà nella sua denotazione simbo- 


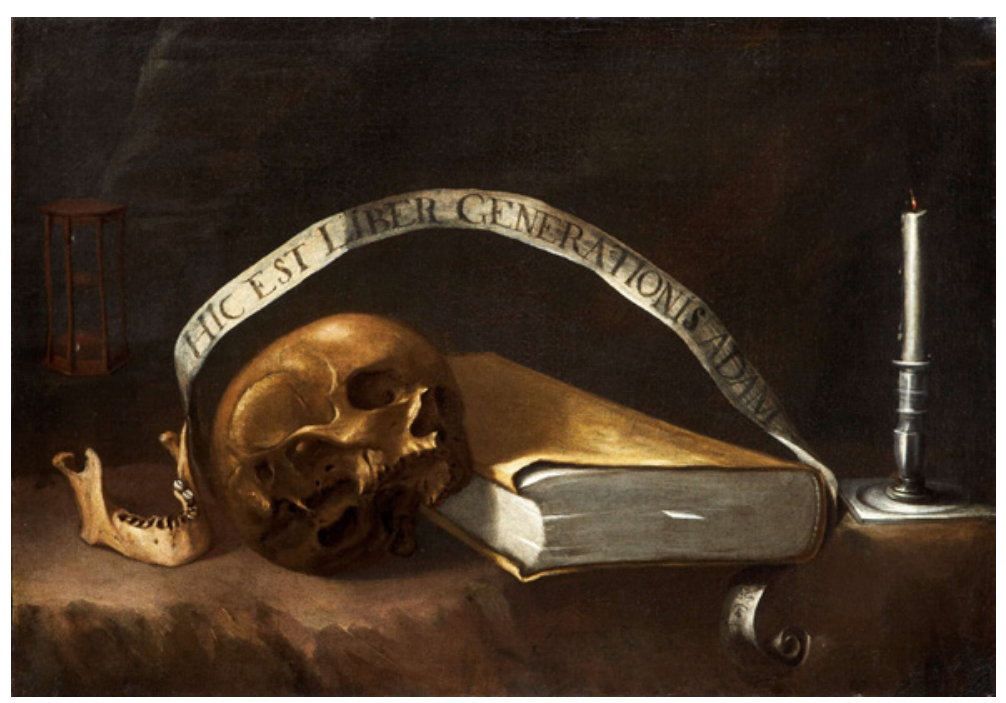

Figura 1 Vanitas attribuita a Juan Francisco Carrión (1672). Olio su tela, $55 \times 77 \mathrm{~cm}$. Madrid, Galería Caylus. Wikimedia Commons @ Public domain

lica allontanandosi dal concetto di uso. La cosa, nelle lingue romanze, è contrazione del latino causa (a questo riguardo si veda Bodei 2009). La cosa/causa è ciò che riteniamo capace di coinvolgerci nella sua difesa o nella sua distruzione. Questo passaggio da una dimensione di utilità a una rappresentazione simbolica è stato ampiamente studiato da Krzysztof Pomian (Pomian 2007). A partire dall'argomentazione di Pomian potrebbe codificarsi questa semantica con il binomio uso/significato. La mediazione tra il visibile e l'invisibile, che può essere concessa al libro, ha portato non solo alla conservazione ma anche alla sua distruzione perché precisamente le cose che sono state caricate di una dimensione semiofora saranno difese da alcuni e bersagliate da altri. Ancora una volta l'attribuzione del significato è radicata nell'osservatore, vale a dire nel ricettore della comunicazione, e non nella cosa né nell'emittente del messaggio. Paradossalmente quegli oggetti consacrati all'uso quotidiano conosceranno, normalmente, solo il logorio a causa del loro utilizzo. Questo connotato semioforo sembrerebbe allontanarsi dall'osservazione contemporanea del libro antico o dei materiali storici d'archivio, dove predomina principalmente la semantica di mercato costituita dal binomio scarsità/abbondanza.

Alla stregua di questa osservazione si è cominciato ad associare a questi beni la preoccupazione per la loro valorizzazione. Questo neologismo diventa di uso comune nel secolo XIX, proveniente dal fran- 
cese valorisation, e trova il suo ambito originario nell'economia. Questo utilizzo è stato definito da Charles Gide come:

hausse factice dans la valeur marchande d’une denrée provoquée au moyen de manœuvres économiques. (Gide 1919, 154)

In questo senso la valorizzazione è vista come il risultato di un'operazione fittizia destinata a dare valore a una merce che, per la sua scarsità provocata, ne aumenta il prezzo. Questo senso di 'operazioni fittizie' si ritrova anche nell'uso che Sigmund Freud, negli stessi anni, fa del termine in quanto sopravalutazione del pensiero in rapporto alla realtà (Freud 1933, 119).

Allo storico può interessare quest'alternanza valutativa in quanto indicatore di mutamenti o di possibili evoluzioni sociali. I gesuiti del Collegio Romano, prima di abbandonare la sede (1773), in seguito alla soppressione dell'Ordine, nascosero una importante quantità di libri manoscritti, stampati e altri oggetti, in uno spazio posteriormente murato. In occasione del ritrovamento del 'ripostiglio', Bartolomeo Podestà, primo bibliotecario della Biblioteca Nazionale Centrale di Roma Vittorio Emanuele, che si era costituita tra le mura del Collegio, ritenne che alcune cose erano di valore, mentre di altre

non si potrebbe spiegare perché fossero nascoste, se non forse per la fretta o pel turbamento d'animo con cui fu condotta l'operazione [...] non si pensò a nascondere volumi e opuscoli di un valore incontestabile bibliografico, quando si fece di manoscritti e di carte inconcludenti. (Podestà 1878, 229)

Quasi la metà del patrimonio del nostro Archivio è rappresentato proprio da queste 'carte inconcludenti' che noi conserviamo con grande cura.

A partire da queste considerazioni potrebbe definirsi la valorizzazione come l'attribuzione di un valore frutto di una selezione basata su determinate osservazioni che utilizzano distinzioni. Negli ultimi anni, abbiamo cercato di stabilire un certo rapporto tra questi principi teorici ed alcune pratiche che sono state attuate alla luce di questo concetto di valorizzazione.

Il residuo patrimoniale dell'Archivio Storico della Pontificia Università Gregoriana che intendiamo conservare e tramandare alle generazioni future va considerato in un determinato paradigma socioculturale nel quale s'inseriscono le nostre pratiche di valorizzazione.

Max Horkheimer e Theodor Adorno, nella Dialettica dell'Illuminismo (Horkheimer, Adorno 2010), hanno riflettuto riguardo la correlazione che si stabilisce tra cultura e società. Il loro concetto operativo è quello delle industrie culturali. Per Horkheimer e Adorno ciò che producono queste industrie culturali è una vera e propria 'bar- 
barie estetica', tendono a vietare l'attività intellettuale dello spettatore/consumatore, paralizzando e alienando per imporre 'l'obbediente accettazione della gerarchia sociale'. Il risultato è l'aumento di una 'crassa incultura, rozzezza e stupidità'. In questo modo la cultura perde la sua dimensione critica per trasformarsi in una fabbrica del consenso. Libri e documenti, spesso non hanno tutte le caratteristiche per soddisfare certe aspettative di visibilità e sono ostici per un consumo immediato, la lettura richiede molto tempo e le mostre tendono a innescare processi di velocizzazione.

\section{Oltre la digitalizzazione}

La digitalizzazione, come le mostre, spesso è presentata come il modo di avvicinare certi contenuti ritenuti di nicchia al grande pubblico, fenomeno identificato con la divulgazione. Questo concetto inclusivo, come ogni inclusione, stabilisce una distinzione tra gli esperti e il vulgo: democratizzando istituisce una gerarchia. In questo modo, esperto e vulgo condividono un destino simile. Da una parte molte pratiche divulgative, che si avvicinano a iniziative di marketing, non assicurano di per sé la fruizione del patrimonio. Essa infatti implica percorsi di comprensione lunghi e complessi per rendere avvicinabili opere che malgrado la loro esposizione o digitalizzazione rimangono incomprensibili. Venendo meno ogni istanza critica, infatti, il vulgo sarà espropriato delle possibilità di esercitare su quel materiale operazioni conoscitive che implichino un vero guadagno di sapere. La divulgazione potrebbe inserirsi in una serie di processi che mirano a consolidare la proletarizzazione intesa come un dis-apprendimento. Dall'altra parte, la frammentazione del sapere, a partire dall'evoluzione delle scienze, è un altro segno che accompagna il sorgere della modernità.

Uno dei risultati di questa insularità dei saperi è la nascita e lo sviluppo della figura dell'esperto. L'esperto si presenta come un mediatore tra un sapere e coloro che ne sono privi. L'esperto padroneggia un linguaggio e mette in moto una pratica regolatrice. Spesso la sua competenza è accompagnata da un'autorità che a volte si esprime in un magistero.

La complessità del reale, però, non corrisponde alla tassonomia delle specializzazioni; capita perciò che l'esperto debba intervenire in questioni che esulano dal suo sapere specialistico ed è in queste situazioni che più che la competenza specialistica vale l'autorità del luogo sociale in cui è inserito. A partire da questo 'abuso di sapere' si arriverà al paradosso di un'autorità a cui

viene attribuito un sapere che le manca proprio là dove si esercita. (de Certeau 2010, 35) 
Così esperti e vulgo sembrano talvolta coincidere nella incapacità di osservare la complessità ed elaborare una descrizione adeguata, per porre domande, valutare rischi e opportunità.

Sempre più spesso biblioteche e archivi promuovono campagne di digitalizzazione con il preteso obiettivo di portare il patrimonio bibliografico e archivistico a un numero di utenti del web sempre più grande. Sovente tuttavia questi sforzi, che implicano grandi investimenti di risorse economiche e umane, non riescono a produrre conoscenza all'interno di una comunità di ricercatori, limitandosi a costituire magnifiche gallerie d'immagini. Nel caso dell'APUG, invece, oltre a soddisfare eventuali richieste di riproduzione per i ricercatori, le campagne di digitalizzazione sono concepite come un primo passo per migliorare la fruizione dei documenti. Affinché l'immagine digitale - che non potrà mai sostituire l'originale - produca conoscenza, essa va accompagnata da metadati, trascrizioni e annotazioni. In questo modo, la nostra preoccupazione si è spostata dal cercare di giustificare l'esistenza dell'archivio incrementando la quantità dei flussi dei lettori/visitatori, a stabilire reti di collaboratori che generino conoscenza. L'inclusione di una maggior quantità di pubblico non necessariamente determina una maggiore partecipazione che possa garantire una fruizione ma, al contrario, l'essere seduto al tavolo da gioco e perfino distribuire le carte potrebbe essere uno dei modi per lasciar le regole del gioco invariate.

\section{Ciò che mostriamo}

Come si potrebbe dedurre da alcune affermazioni precedenti, alla società non appartiene il libro in quanto tale, ma le comunicazioni che si fanno sul suo conto. Lo stesso potremmo dire della tecnologia. Anche essa è esterna al sistema società, si trova, per così dire, nel suo ambiente. Con ciò non si vuole negare la profonda influenza sociale della tecnologia così come fu, secoli fa l'apparizione del libro che incrementò una serie di dinamiche evolutive innescate a partire del XVI secolo.

Allo stadio in cui ci troviamo dello sviluppo tecnologico, la tecknè si costituisce come un sistema che opera allopoieticamente, vale a dire, non autopoieticamente come il sistema sociale che è operativamente chiuso. Questo ci dà la possibilità di interferire direttamente nelle sue operazioni. Detto altrimenti, c'è l'opportunità di disegnare delle architetture digitali che abbassino i livelli di entropia che la rete genera, in virtù della quale i saperi sono automatizzati e omologati, per cercare di costruire invece delle biforcazioni neghentropiche che favoriscano la costruzione di conoscenza a partire della collaborazione (Schrödinger 1995; Stiegler 2019).

Per inseguire questa strada l'APUG, nel 2017 ha realizzato la piattaforma GATE (Gregorian Archives Texts Editing), basata sul software 
gratuito e open source MediaWiki e arricchita con estensioni ad hoc, quali ad esempio Semantic MediaWiki. Le modalità cooperative su cui si basa GATE (gate.unigre.it) riflettono il presupposto che i 'saperi', intesi non solo come conoscenza ma come saper fare e saper vivere, si costituiscono in maniera collaborativa. Questo progetto non si configura in modo interdisciplinare, quanto piuttosto in forma transdisciplinare: integrando cioè diversi punti di vista nell'osservazione di complessità superiori alle stesse discipline.

Normalmente la rete è presentata e utilizzata come uno spazio di consumo; non pensiamo solo ai consumatori cooptati dal marketing, ma anche alla fruizione di news o di dati (siano essi tratti da un catalogo di biblioteca o da un motore di ricerca come Google). Il World Wide Web, che è apparso nel dominio pubblico dal 30 aprile 1993, fu concepito da un gruppo di ricercatori del CERN nel 1989.

Lo scopo della costituzione di una tale rete era avere uno spazio affinché informatici, fisici, biologi e altri scienziati potessero arricchire i propri saperi con la pratica della controversia. Con la crescente automazione generalizzata dei dati, questo obiettivo è divenuto utopico. Infatti, secondo la ratio dell'1\%, gli autori di nuovi contenuti rappresentano una percentuale minima, mentre la maggior parte degli utenti del web guarda e prende, cioè consuma.

GATE, al contrario, si propone come uno spazio di ricerca collaborativa che si concentra in modo particolare sul patrimonio conservato in APUG. Tra i suoi obiettivi c'è l'individuazione del cambiamento di alcuni concetti nel corso del secolo XVII. Dall'osservazione di tali cambiamenti si può inferire un processo evolutivo nel sistema sociale a partire dalla modernità incipiente. Attraverso una lettura storicizzata della società della prima modernità, GATE intende contribuire a una descrizione adeguata della società odierna. La comprensione della nostra società inizia dal rilevamento delle differenze con i tempi passati e del modo in cui essa si è sviluppata da strutture precedenti. A partire da queste differenze si potranno comprendere le opportunità della nostra società, la sua struttura e i pericoli che la minacciano.

Questa premessa teorica consente di attribuire un valore ai dati selezionati e osservati, per trarne informazioni e creare conoscenza. Per descrivere un sistema complesso come quello della società nella prima modernità sono stati scelti alcuni studi di caso. Questo metodo di lavoro considera determinanti non gli aspetti biografici dei personaggi storici, ma i documenti.

Le fonti sono considerate in quanto forme discorsive contestualizzate e non come risultato di un'esperienza o di una percezione individuale. Pertanto, il 'supporto' della fonte non si trova nell'individuo ma nel sistema sociale che lo ha prodotto.

Ad esempio, considerando la produzione a stampa e manoscritta di Athanasius Kircher (1602-80), del quale l'Archivio possiede all'incirca 
Martín M. Morales

L'archivio della Pontificia Università Gregoriana (APUG) e la costruzione di un oltre

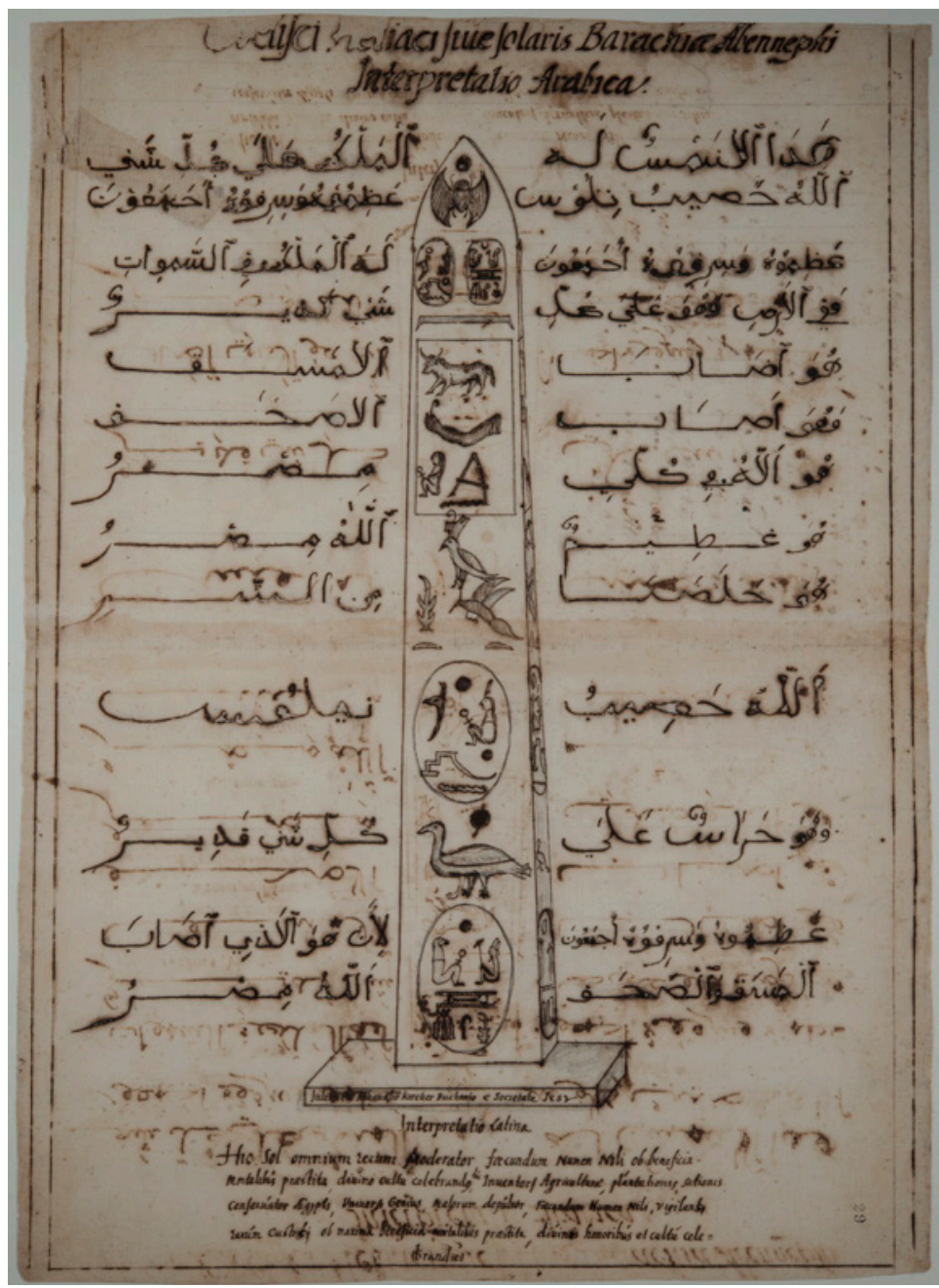

Figura 2 Obelisci Heliaci sive solaris Barachiae Abennephi Interpretatio Arabica, APUG 830 cc. 28-29. Disegno a inchiostro su carta, $276 \times 380 \mathrm{~mm}$ (c) Archivio Storico della Pontificia Università Gregoriana 
3.000 lettere diverse oltre a opere manoscritte, è possibile tracciare l'evoluzione di alcuni concetti chiave, quali admiratio, curiositas o novitas. Oltre al trattamento dei testi di Kircher e della sua cerchia, è stata intrapresa una raccolta bibliografica che ad oggi conta oltre 650 titoli, lavoro che consentirà di analizzare le osservazioni condotte sull'opera di Kircher nel corso dei secoli. Il restauro dei disegni di tre obelischi kircheriani ${ }^{1}$ è stato il primo passo per procedere poi alla loro digitalizzazione e inserimento nella piattaforma per iniziare il processo di trascrizione e annotazione [fig. 2]. Questo potrebbe essere un esempio di come le operazioni di restauro, digitalizzazione e analisi del testo possano integrarsi come fasi di un unico processo.

Un altro studio di caso presentato sulla piattaforma riguarda la produzione di Roberto Bellarmino. L'analisi della sua corrispondenza (2.674 lettere) consentirà, ad esempio, di rintracciare la semantica concettuale rappresentata in termini come 'eresia' o 'errore', o di valutare l'impatto della stampa e i diversi aspetti del mondo del libro nella diffusione e ricezione del sapere.

La dimensione collaborativa che presuppone la piattaforma GATE trova le sue radici nella convinzione che i saperi si creino a partire dalla condivisione, in uno spazio di autonomia e libertà. La tecnologia potrebbe, oggi, prendere le mosse da questa dimensione collaborativa. Spesso, però, la rete, che sembrerebbe il mito di molte organizzazioni, vieta 0 , quanto meno, è concepita per controllare le disseminazioni, le possibili e infinite biforcazioni del pensiero, dove precisamente si genera la conoscenza. La censura ha saputo, lungo la storia, gettare tante reti. GATE, in qualche modo, si riallaccia al concetto di skholè che indica ozio, svago, in quanto temporalità libera e non finalizzata alla produzione né all'utilità ma alla pratica dello studio per se stesso. La skholè, è l'alternativa dell'entertainment, che è l'obiettivo delle industrie culturali. Contrariamente a queste, tende a provocare, invitando alla partecipazione creativa, alla responsabilità e all'impegno.

Questa piattaforma presuppone, inoltre, uno spazio accademico così come tracciato da Immanuel Kant nel Conflitto delle facoltà. Kant prevedeva, oltre alle Università, le accademie ove i dilettanti nei loro laboratori (accademie) si occupassero, per il semplice piacere di farlo, dell'ampliamento e diffusione del sapere, senza ubbidire a regole o norme pubbliche.

Da questo consegue che l'utente di GATE non sia principalmente un consumatore che si aggira nella rete ma un amatore, un collaboratore. A questo collaboratore si dà l'opportunità non solo di attingere a dei contenuti ma di crearli, di completarli e di discuterli, superando la miseria simbolica di esprimersi con un like o con un'emoticon di occasione.

1 https://gate.unigre.it/mediawiki/index.php/Obelisks_drawings. 


\section{Bibliografia}

Bodei, R. (2009). La vita delle cose. Roma-Bari: Laterza.

de Certeau, M. (2010). L'invenzione del quotidiano. Roma: Edizioni Lavoro.

Freud, S. (1933). Totem et tabou: interprétation par la psychanalyse de la vie sociale des peuples primitifs. Paris: Payot.

Gide, C. (1919). Cours d'économie politique. Paris: Recueil Sirey.

Horkheimer, M.; Adorno, T. (2010). Dialettica dell'Illuminismo. Trad. di R.Solmi. Torino: Einaudi.

Luhmann, N. (2005). Organizzazione e decisioni. Trad. di G. Corsi. Milano: Mondadori Bruno.

Podestà, B. (1878). «Oggetti trovati in un ripostiglio annesso ai locali della biblioteca Vittorio Emanuele». Bollettino ufficiale del Ministero della pubblica istruzione, 4(2), 227-9.

Pomian, K. (2007). Collezionisti, amatori e curiosi Parigi-Venezia XVI-XVIII secolo. Trad. di G. Arnaldi, D. Modonesi, M. Romano, D. Tortorella. Milano: II Saggiatore.

Reale, G. (2009). Introduzione, traduzione e commentario della "Metafisica" di Aristotele. Milano: Bompiani.

Schrödinger, E. (1995). Che cos'è la vita? La cellula vivente dal punto di vista fisico. Trad. di M. Ageno. Milano: Adelphi.

Stiegler, B. (2019). La società automatica. Trad. di S. Baranzoni, I. Pelgreffi, P. Vignola. Milano: Meltemi. 

Il volume raccoglie gli Atti del seminario Oltre le mostre.

Proposte per una diversa valorizzazione del patrimonio archivistico e librario organizzato dall'Associazione Italiana dei Conservatori e Restauratori degli Archivi e delle Biblioteche (AICRAB) a Napoli il 28 febbraio 2020 presso la Biblioteca Fra Landolfo Caracciolo, nel complesso monumentale di San Lorenzo Maggiore. I contributi riflettono sulle modalità attraverso le quali il patrimonio archivistico e librario viene promosso al grande pubblico. Le opportunità di valorizzazione sono oggi potenziate dalla dimensione digitale e dal web che, se da un lato costituiscono un incremento delle risorse a disposizione, dall'altro pongono molteplici interrogativi insiti nella propria natura.

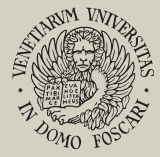

Università

Ca'Foscari

Venezia

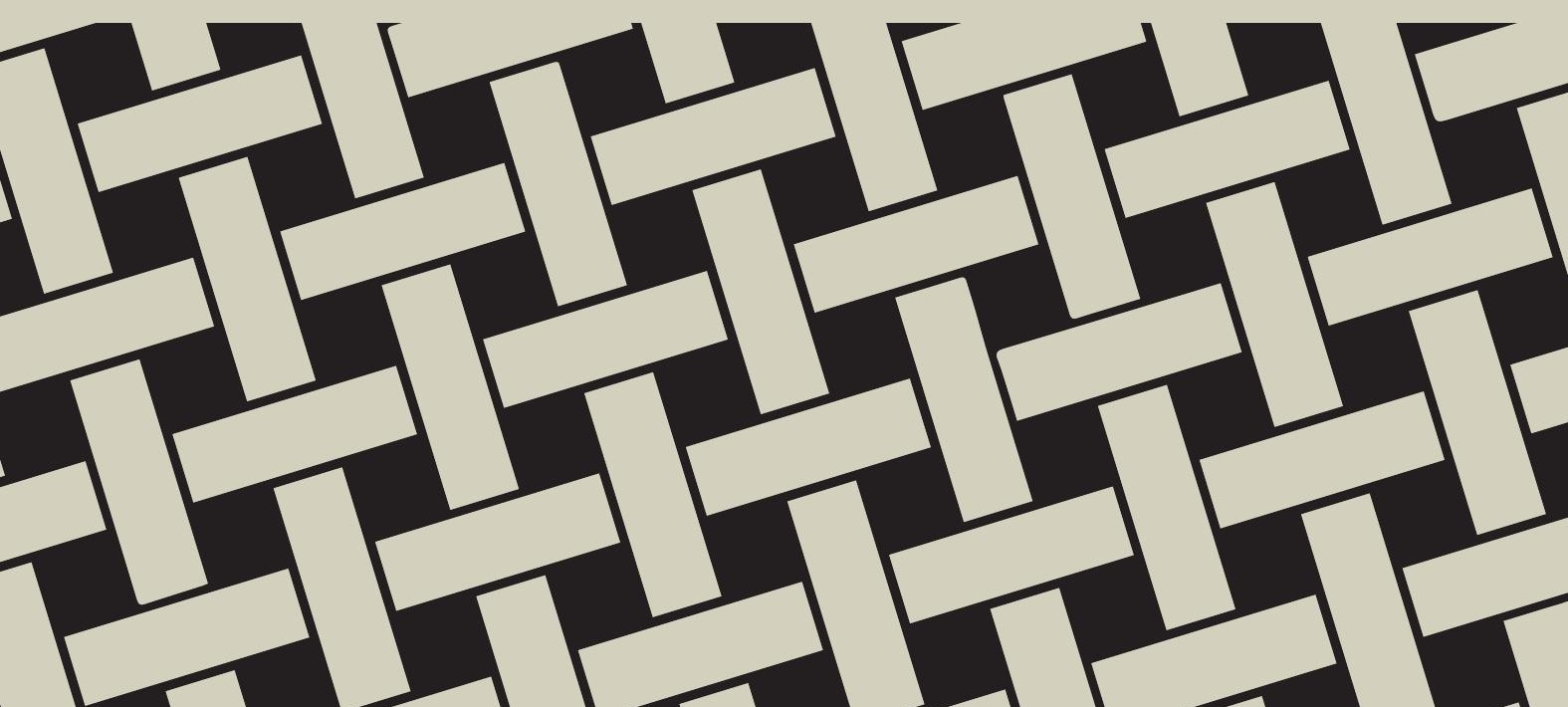

